\title{
STABLE MAPS AND BRANCHED SHADOWS OF 3-MANIFOLDS
}

\author{
MASAHARU ISHIKAWA AND YUYA KODA
}

\begin{abstract}
Turaev's shadow can be seen locally as the Stein factorization of a stable map. In this paper, we define the notion of stable map complexity for a compact orientable 3-manifold bounded by (possibly empty) tori counting, with some weights, the minimal number of singular fibers of codimension 2 of stable maps into the real plane, and prove that this number equals the minimal number of vertices of its branched shadows. In consequence, we give a complete characterization of hyperbolic links in the 3 -sphere whose exteriors have stable map complexity 1 in terms of Dehn surgeries, and also give an observation concerning the coincidence of the stable map complexity and shadow complexity using estimations of hyperbolic volumes.
\end{abstract}

2010 Mathematics Subject Classification: 57R45; 57M27, 57N70, 58K15

Keywords: stable map; complexity; branched shadow; hyperbolic volume

\section{INTRODUCTION}

The stable maps play an important role in the study of smooth manifolds. They are especially used for obtaining topological information of the source manifold from the types of their singularities and singular fibers. A typical example is the usage of critical points of a Morse function, which is a stable map of a manifold into the real line. In this paper, we deal with stable maps from a closed orientable 3-manifold $M$ to the real plane $\mathbb{R}^{2}$. As is well-known, the set $S(f)$ of singular points of a stable map $f: M \rightarrow \mathbb{R}^{2}$ consists of definite fold points, indefinite fold points and cusp points, see [23, 24]. In [23] Levine showed that the cusp points of each stable map can be eliminated by a homotopical deformation, which implies that every 3-manifold admits a stable map into $\mathbb{R}^{2}$ without cusp points. We note that when $f: M \rightarrow \mathbb{R}^{2}$ is a stable map without cusp points, $S(f)$ forms a link in $M$ and $\left.f\right|_{S(f)}$ is an immersion with only normal crossings. A crossing of $f(S(f))$ is said to be non-simple if only one of the connected components of its preimage contains singularities. Burlet-de Rham [5] showed that the 3-manifolds admitting a stable map with only definite fold points are either the 3-sphere or connected sums of $S^{2} \times S^{1}$. Saeki [36] generalized this result showing that the 3-manifolds admitting a stable map with neither non-simple crossings nor cusp points are graph manifolds, and vice versa. It follows from Saeki's work that we need non-simple crossings to construct a stable map of a hyperbolic 3-manifold into the plane. Hence it is natural to ask how many non-simple crossings a hyperbolic 3-manifold needs to have and where is the position of

The first-named author is supported by the Grant-in-Aid for Scientific Research (C), JSPS KAKENHI Grant Number 25400078.

The second-named author is supported by Japan Society for Promotion of Science (JSPS) Postdoctoral Fellowships for Research Abroad. 
the singular fibers. Costantino-Thurston [12] and Gromov [15] gave, independently, a linear lower bound of the number of non-simple crossings of stable maps that a closed 3-manifold admits in terms of Gromov norm.

On the other hand, Turaev [41, 42] introduced the notion of shadows of 4 and 3manifolds in his great deal of study on quantum invariants. Roughly speaking, a shadow of a compact oriented 4-manifold $W$ is a special kind of 2-dimensional polyhedron (almost-special polyhedron) $P$ embedded in $W$ in a locally flat way so that $W$ collapses onto $P$. Then $P$ is also called a shadow of the 3 -manifold $\partial W$. Shadows provide a combinatorial presentation of both the 4 and 3-manifolds. In fact, by means of shadows, we can reconstruct a 4 and 3-manifold in a unique way from an almost-special polyhedron $P$ equipped with a suitable coloring of the regions of $P$ by half integers, which is called a gleam. Shadows provide important topological properties for 4 and 3-manifolds as well as being an interesting notion for the study of quantum topology. We refer the reader to Costantino-Thurston [12] for triangulations of 4 and 3-manifolds, Costantino [9, 10] for Stein structure, $\operatorname{Spin}^{c}$ structure and complex structure of 4-manifolds and Martelli [26] for a classification of 4-manifolds admitting shadows without vertices. See also the survey paper [8] by Costantino. Shadows can be defined also for a link in a compact orientable 3 -manifold with (possibly empty) boundary consisting of tori. In general, shadows are defined for a trivalent graph in each compact, orientable 3-manifold, however, we consider only in the above case.

The Stein factorization of a map is the space of the connected components of its fibers. This is a powerful tool for the study of stable maps, because, in this case, the Stein factorization has particularly simple local shapes. In fact, in each of the papers [5], 36] and [24] mentioned earlier, Stein factorization played an important role. In [12], Costantino-Thurston revealed a strong relation between the Stein factorizations of stable maps of 3-manifolds into the plane and the shadows of the manifolds. In this paper, we study the detailed relation between branched shadows and stable maps of a 3-manifold (and a link in it). Here, a branched shadow is a shadow equipped with an orientation of each of their regions in a certain admissible way.

Let $M$ be a compact, orientable 3-manifold with (possibly empty) boundary consisting of tori and $L$ a (possibly empty) link in $M$. We call the minimal number of vertices in any of branched shadows of $(M, L)$ the branched shadow complexity of $(M, L)$, and we denote it by $\operatorname{bsc}(M, L)$. We can define another kind of complexity of $(M, L)$ using the theory of stable maps. Here we note that the definition of stable maps can be naturally generalized for the above pair $(M, L)$. In this paper, we call this map an $S$-map. See Section 1.2 for details. For an S-map of $(M, L)$ into $\mathbb{R}^{2}$, the singular fibers over nonsimple crossings are divided into two types, types $\mathrm{II}^{2}$ and $\mathrm{II}^{3}$, according to their shapes as shown in Figure 1, see Saeki [37. We denote by $\mathrm{II}^{2}(f)$ and $\mathrm{II}^{3}(f)$ the sets of singular fibers of types $\mathrm{II}^{2}$ and $\mathrm{II}^{3}$, respectively, of an S-map $f:(M, L) \rightarrow \mathbb{R}^{2}$. We call the minimal number of the "weighted sums" $\left|\mathrm{II}^{2}(f)\right|+2\left|\mathrm{II}^{3}(f)\right|$ for S-maps $f:(M, L) \rightarrow \mathbb{R}^{2}$ the stable map complexity of $(M, L)$, and we denote it by $\operatorname{smc}(M, L)$. The first main result of this paper is the following: 


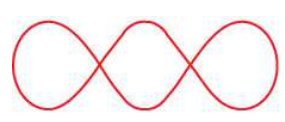

(i)

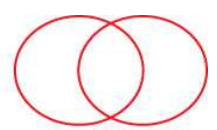

(ii)

Figure 1. (i) A singular fiber of type $\mathrm{II}^{2}$; (ii) A singular fiber of type $\mathrm{II}^{3}$.

Theorem 2.2. Let $M$ be a compact, orientable 3-manifold with (possibly empty) boundary consisting of tori and $L$ a (possibly empty) link in $M$. Then we have $\operatorname{bsc}(M, L)=$ $\operatorname{smc}(M, L)$.

By Costantino-Thurston [12], the inequality $\operatorname{sc}(M, L) \leqslant \operatorname{smc}(M, L)$ holds, where $\operatorname{sc}(M, L)$ is the shadow complexity of $(M, L)$, that is, the minimal number of vertices in any of its shadows. Since a shadow constructed from a stable map in their way is branchable, we have the inequality $\operatorname{bsc}(M, L) \leqslant \operatorname{smc}(M, L)$ immediately. To get the other inequality, we construct from a given branched shadow a stable map in 2 steps: In the first step, we construct an S-map on the submanifold of $M$ corresponding to a neighborhood of the singularities of the branched shadow so that its Stein factorization is isomorphic to the neighborhood. In the second step, we extend this map to whole of $M$ without creating non-simple crossings. This theorem implies branched shadows of $(M, L)$, which is a purely combinatorial object, determine the minimal number of the weighted sums of non-simple crossings of stable maps of $(M, L)$, which is a purely differential value. It is worth noting that in case where we consider a stable map of a closed orientable 3 -manifold $M$ into $\mathbb{R}$, instead of $\mathbb{R}^{2}$, the minimal number of critical points is determined by some specific branched spines of $M$, see Remark 2.3 (3).

Due to Theorem 2.2, we can use branched shadows to study the behavior of stable map complexities under several operations of 3-manifolds. In fact, the subadditivities of branched shadow complexities under both connected sum and torus sum are obtained by easy combinatorial constructions and these provide immediately the same properties for stable map complexities (cf. Corollaries 2.6, 2.8). It follows immediately that stable map complexities do not decrease under Dehn filling (cf. Corollary 2.10). This behavior is similar to that of the hyperbolic volumes shown by Thurston [39].

In the remaining part of the paper, we discuss more applications of Theorem 2.2. First, we construct a stable map for a link in the 3-sphere using a branched shadow, and introduce a way to determine the configuration of its singular fibers. This construction and the result for Dehn filling that mentioned earlier yield the following:

Corollary 3.3. Let $M$ be a closed orientable 3-manifold obtained from $S^{3}$ by surgery along a link $L$. Then there exists a stable map $f: M \rightarrow \mathbb{R}^{2}$ without cusp points such that $\left|\mathrm{II}^{2}(f)\right| \leqslant \operatorname{cr}(L)-2$ and $\mathrm{II}^{3}(f)=\emptyset$, where $\operatorname{cr}(L)$ is the crossing number of $L$.

In [18, Kalmár-Stipsicz described the upper bounds of the minimal numbers of simple crossings, $\mathrm{II}^{2}(f), \mathrm{II}^{3}(f)$, etc., for stable maps that a closed 3-manifold $M$ admits in terms of properties of a surgery diagram of $M$. The above corollary provides better upper bounds for $\left|\mathrm{II}^{2}(f)\right|,\left|\mathrm{II}^{3}(f)\right|$ than theirs. 
Next, developing the technique in the above construction, we characterize the hyperbolic knots in $S^{3}$ having branched shadow complexity 1 as follows:

Theorem 4.6. Let $L$ be a hyperbolic link in $S^{3}$. Then $\operatorname{bsc}\left(S^{3}, L\right)=1$ if and only if the exterior of $L$ is diffeomorphic to a 3-manifold obtained by Dehn filling the exterior of one of the six links $L_{1}, L_{2}, \ldots, L_{6}$ in $S^{3}$ along some of (possibly none of) boundary tori of its exterior, where $L_{1}, L_{2}, \ldots, L_{6}$ are illustrated in Figure 2.

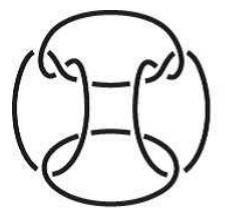

$L_{1}$

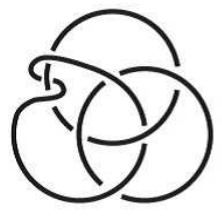

$L_{4}$

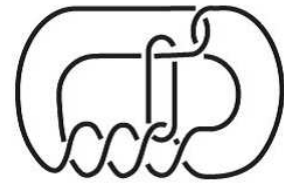

$L_{2}$

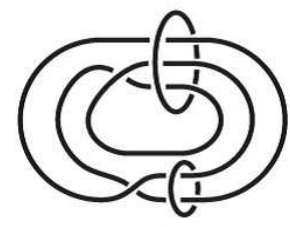

$L_{5}$

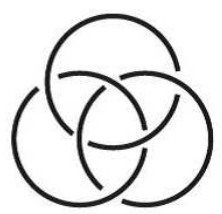

$L_{3}$

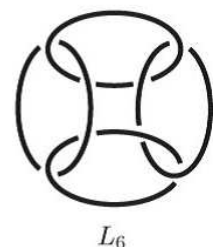

Figure 2. The links $L_{1}, L_{2}, \ldots, L_{6}$ in $S^{3}$.

Each of the links $L_{1}, L_{2}, \ldots, L_{6}$ of this theorem is a hyperbolic link having the volume $2 V_{\text {oct }}$, where $V_{\text {oct }}=3.66 \ldots$ is the volume of the ideal regular octahedron. See Costantino-Thurston [12, Proposition 3.33]. We note that by Agol-Storm-Thurston [3, Theorem 9.1], the link $L_{1}$ is a minimal volume hyperbolic link that contains a meridional incompressible planar surface. See also Agol [1, Example 3.3]. In [43] Yoshida proved that the complement of $L_{6}$ is the minimal volume orientable hyperbolic 3-manifold with 4 cusps.

Theorem 4.6 can be restated in terms of singular fibers of stable maps as follows:

Corollary 4.8. Let $L$ be a hyperbolic link in $S^{3}$. Then there exists a stable map $f$ : $\left(S^{3}, L\right) \rightarrow \mathbb{R}^{2}$ without cusp points such that $\left|\mathrm{II}^{2}(f)\right|=1$ and $\mathrm{II}^{3}(f)=\emptyset$ if and only if the exterior of $L$ is diffeomorphic to a 3-manifold obtained by Dehn filling the exterior of one of the six links $L_{1}, L_{2}, \ldots, L_{6}$ in Theorem 4.6 along some of (possibly none of) boundary tori of its exterior.

We also describe the configuration of the singular fiber of the above S-map for $E\left(L_{i}\right)$, $i=3,4,5,6$ in Corollary 4.9,

Finally, we discuss relation between stable map complexities and hyperbolic volumes. Costantino-Thurston 12 showed the inequality $\|M\| V_{\text {tet }} \leqslant 2 \operatorname{sc}(M) V_{\text {oct }}$ for any 3 -manifold $M$, where $\|M\|$ is the Gromov norm of $M$ and $V_{\text {tet }}=1.01 \ldots$ is the volume of the ideal regular tetrahedron. The same argument together with Theorem 2.2 gives the following inequality relating stable map complexities and Gromov norms:

$$
\|M\| V_{\text {tet }} \leqslant 2 \operatorname{smc}(M) V_{\text {oct }} .
$$


In particular, if $M$ is hyperbolic then the inequality $\operatorname{vol}(M) \leqslant 2 \operatorname{smc}(M) V_{\text {oct }}$ holds. The lower bound of $\operatorname{vol}(M)$ by $\operatorname{smc}(M)$ can also be given by using branched, special shadows, where a branched shadow $P$ is special if $P$ is stratified by its singularities as a $\mathrm{CW}$ complex. We note that every closed orientable 3 -manifold admits a branched, special shadow by the moves described in [42, 7]. By applying Futer-Kalfagianni-Purcell [14, Theorem 1.1] to our situation, we have the inequality

$$
2 \operatorname{smc}(M) V_{\text {oct }}\left(1-\left(\frac{2 \pi}{\operatorname{sl}(P)}\right)^{2}\right)^{3 / 2} \leqslant \operatorname{vol}(M),
$$

where $M$ is a closed orientable hyperbolic 3 -manifold with special shadow $P$, and $\operatorname{sl}(P)$ is a certain positive real number determined by the gleam and the topological data of $P$, which corresponds to the minimal slope length of Dehn fillings. We require $\operatorname{sl}(P)>2 \pi$ in the above inequality, see Section 5 for details. From these inequalities we have the following result that concerns the coincidence of shadow complexities, branched shadow complexities and stable map complexities.

Theorem 5.2. Let $M$ be a closed orientable 3-manifold, and let $P$ be a branched, special shadow of $M$. If $\operatorname{sl}(P)>2 \pi \sqrt{2 c(P)}$, then we have $\operatorname{sc}(M)=\operatorname{bsc}(M)=\operatorname{smc}(M)=c(P)$.

It seems to be hard to compute the shadow complexities, branched shadow complexities and stable map complexities by just looking at their definitions, because we need to consider the minimal of infinitely many shadows, branched shadows and weighted sums of non-simple crossings, respectively. Moreover, it seems to be too optimistic to expect that the shadow complexities coincide with the branched shadow complexities, since there exist infinitely many shadows that are not branchable. However, Theorem 5.2 suggests that these three kinds of complexities coincide for many cases.

This paper is organized as follows. In Section 1 we review the definitions of branched shadows and stable maps. In Section 2, we provide the proof of Theorem 2.2, and several direct corollaries of it. In Section 3, we introduce a way to construct a stable map for each link $L$ in the 3 -sphere $S^{3}$, and describe the configuration of the fibers of the map. Section 4 is devoted to a characterization of the hyperbolic knots in $S^{3}$ having the stable map complexity 1 and its applications. In Section 5 , we discuss relation between stable map complexities and hyperbolic volumes.

Throughout the paper, we will work in the smooth category unless otherwise mentioned.

\section{Preliminaries}

1.1. Shadows and branched shadows of 3-manifolds. A compact topological space $P$ is called an almost-special polyhedron if every point of $P$ has a regular neighborhood homeomorphic to one of the five local models shown in Figure 3. A point whose regular neighborhood is shaped on the model (iii) is called a true vertex of $P$, and we denote the set of true vertices of $P$ by $V(P)$. The set of points whose regular neighborhood are shaped on the models (ii), (iii) or (v) is called the singular set of $P$, and we denote it 


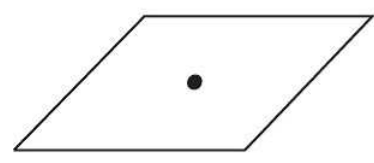

(i)

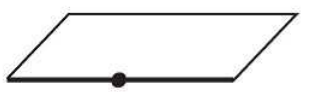

(iv)

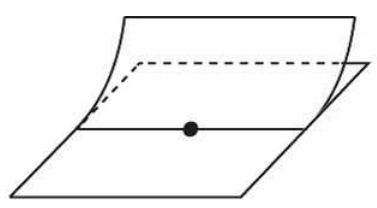

(ii)

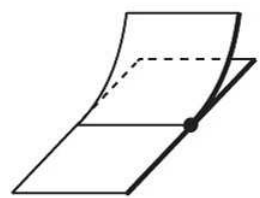

(v)

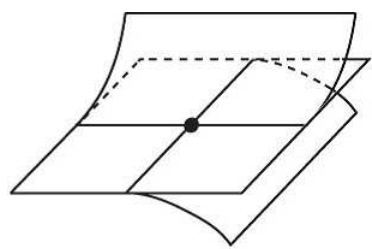

(iii)

FiguRE 3. The local models of an almost-special polyhedron.

by $S(P)$. The set of points whose regular neighborhood are shaped on the models (iv) or (v) is called the boundary of $P$ and we denote it by $\partial P$. A point whose regular neighborhood is shaped on the model $(\mathrm{v})$ is called a boundary-vertex of $P$, and we denote the set of boundary-vertices of $P$ by $B V(P)$. Throughout the paper, we set $c(P)=|V(P)|+|B V(P)|$. The polyhedron $P$ is said to be closed if $\partial P=\emptyset$. Each component of $P \backslash S(P)$ is called a region. A region is said to be internal if it does not touch the boundary of $P$. Otherwise, it is said to be external.

Let $P$ be an almost-special polyhedron. A coloring of $\partial P$ is an arbitrary map from the set of components of $\partial P$ to $\{i, e, f\}$. Then with respect to the coloring, $\partial P$ decomposes into three peaces $\partial_{i} P, \partial_{e} P$ and $\partial_{f} P$. An almost-special polyhedron is said to be boundary-decorated if it is equipped with a coloring of $\partial P$. If $\partial_{f}(P)=\emptyset, P$ is said to be proper.

Definition. Let $M$ be a compact orientable 3-manifold and $L$ a (possibly empty) link in $M$. A boundary-decorated almost-special polyhedron $P$ properly embedded in a compact oriented smooth 4-manifold $W$ is called a shadow of $(M, L)$ if

- $W \backslash P$ is diffeomorphic to $\partial W \times(0,1]$, or equivalently, $W$ collapses onto $P$ after equipping a natural PL structure on $W$;

- $P$ is locally flat, that is, each point $p$ of $P$ has a neighborhood $\operatorname{Nbd}(p ; P)$ that lies in a 3-dimensional submanifold of $W$; and

- $(M, L)=\left(\partial W \backslash \operatorname{Nbd}\left(\partial_{e} P ; \partial W\right), \partial_{i} P\right)$.

When $L=\emptyset$, we say that $P$ is a shadow of $M$ for simplicity.

In [41, 42, Turaev proved that any pair of a compact orientable 3-manifold with no spherical boundary components and a (possibly empty) link in it has a shadow. In [7, 12], the shadow complexity of $(M, L)$, denoted by $\operatorname{sc}(M, L)$, was defined to be the minimal number of true and boundary-vertices in any of its shadows.

Remark 1.1. In Costantino-Thurston [12, Remark 3.19], it is proved that, if $M$ is a compact orientable 3-manifold with boundary consisting of (possibly empty) tori, then 
$\operatorname{sc}(M, L)$ coincides with the minimal number of true vertices in any of shadows of $(M, L)$. Actually, in their paper, $\operatorname{sc}(M, L)$ was defined using only the true vertices.

A branching of an almost-special polyhedron $P$ is an orientation of each region of $P$ such that the orientations on each component of $S(P) \backslash V(P)$ induced by the regions does not coincide. A branching of $P$ allows us to smoothen $P$ as in the local models in Figure 3. We note that even though each region of an almost-special polyhedron $P$ is orientable, $P$ does not necessarily admit a branching. See Ishii [17], Benedetti-Petronio [4] and Petronio [34 for general properties of branched polyhedra.

Definition. Let $M$ be a compact orientable 3-manifold and $L$ a (possibly empty) link in $M$. A shadow $P$ of $(M, L)$ equipped with a branching is called a branched shadow of $(M, L)$.

In [7, Theorem 3.1.7] and [10, Proposition 3.4], Costantino described an algorithmic procedure to obtain a branched shadow from an arbitrary shadow through a finite sequence of moves. As a consequence, any pair of a compact orientable 3-manifold with no spherical boundary components and a (possibly empty) link in it has a branched shadow.

Definition. Let $M$ be a compact orientable 3-manifold and $L$ a (possibly empty) link in $M$. The branched shadow complexity of $(M, L)$, denoted by $\operatorname{bsc}(M, L)$, is the minimal number of true and boundary-vertices in any of its branched shadows. A branched shadow $P$ of $(M, L)$ is said to be minimal if it satisfies $c(P)=\operatorname{bsc}(M, L)$, that is, it contains the least possible number of true and boundary-vertices.

Remark 1.2. At the moment, we do not know whether $\operatorname{bsc}(M, L)$ coincides with the minimal number of only true vertices in any of branched shadows of $(M, L)$ if $M$ is a compact orientable 3-manifold with boundary consisting of (possibly empty) tori. More generally, we do not know whether $\operatorname{sc}(M, L)$ coincides with $\operatorname{bsc}(M, L)$. See Theorem 5.2 in the last section of this paper.

A gleam on an almost-special polyhedron $P$ is a coloring of all the interior regions of $P$ with half integers satisfying a certain condition. We call an almost-special polyhedron $P$ equipped with gleams a shadowed polyhedron. In [41, 42, Turaev showed the following:

(1) If an almost-special polyhedron $P$ is embedded in a compact oriented smooth 4-manifold $W$ so that $P$ is locally-flat, there exists a canonical coloring of the interior regions of $P$ with half integers, that is, we have the canonical gleam on $P$.

(2) (Turaev's reconstruction) From a shadowed polyhedron $P$, we can reconstruct a compact oriented smooth 4-manifold $W$ and an embedding $P \hookrightarrow W$ in a unique way (up to diffeomorphism) so that $W$ collapses onto $P$ and the canonical gleam on $P$ given by the embedding $P \hookrightarrow W$ coincides with the prefixed gleam on $P$.

Here we briefly explain the reconstruction of an oriented 4-manifold from a shadowed polyhedron $P$ only in the case where $\partial P=\emptyset, S(P) \neq \emptyset$ and $S(P)$ is connected. For the detailed and general construction we refer the reader to Costantino [7] (see also Thurston [38] and Costantino-Thurston [12]). Let $P$ be a shadowed polyhedron without boundary such that $S(P)$ is non-empty and connected. The polyhedron $\operatorname{Nbd}(S(P) ; P)$ decomposes into pieces each of which is homeomorphic to a compact surface or one of 
the local models (ii) and (iii) shown in Figure 3. For each piece homeomorphic to the models (ii) or (iii), we consider its 3-dimensional thickening and then glue all these pieces following a natural instruction given by the combinatorial structure of $P$. We note that the resulting 3-manifold $X$ is not necessarily orientable. Let $W_{0}$ be the subbundle of the determinant line bundle over $X$ whose fiber is $[-1,1]$ (after giving an Euclidean metric over this bundle). This is the unique $[-1,1]$ bundle over $X$ whose total space is orientable. Let $R$ be a component of $P \backslash \operatorname{Int} \operatorname{Nbd}(S(P) ; P)$. Let $W_{R}$ be the subbundle of the determinant line bundle over $R \times[-1,1]$ whose fiber is $[-1,1]$. When $R$ is an orientable surface, $W_{R}$ is nothing else but $R \times[-1,1] \times[-1,1]$. The combinatorial structure of $P$ again tells us which part of $\partial W_{R}$, which is a solid torus, will be glued to which part of $\partial W_{0}$, which is also a torus, to obtain the required 4-manifold $W$.

Let $l_{1}, l_{2}, \ldots, l_{k}$ be $k$ simple closed curves constituting $\partial \operatorname{Nbd}(S(P) ; P) \cap R$. For each $i \in\{1,2, \ldots, k\}$, the 3 -dimensional thickening of $\operatorname{Nbd}(S(P) ; P)$ provides an annulus or a Möbius band $B_{i}$ whose core is $l_{i}$. Similarly, the 3-dimensional thickening $R \times[-1,1]$ provides an annulus $A_{i}$ whose core is $l_{i}$. Now we have two "framings" $A_{i}$ and $B_{i}$ of the same simple closed curve $l_{i}$, which lie in the same solid torus after the earlier-mentioned identification. Suppose that $B_{i}$ is obtained from $A_{i}$ by twisting $n_{i}$ times $\left(n_{i} \in(1 / 2) \mathbb{Z}\right)$. See Figure 4. The gleam of the region corresponding to $R$ is the sum $\sum_{i=1}^{k} n_{i}$.

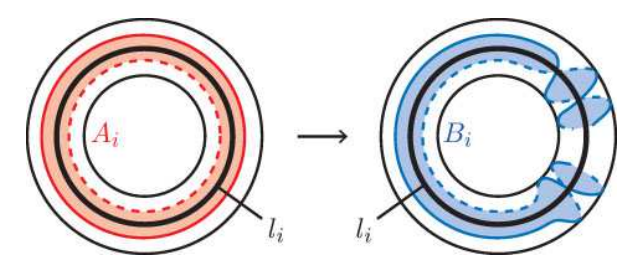

Figure 4. The framings $A_{i}$ and $B_{i}$.

1.2. Stable maps and their Stein factorizations. Let $M$ be a closed orientable 3manifold. Let $f$ be a map of $M$ into an orientable 2-manifold $\Sigma$. We denote by $S(f)$ the set of singular points of $f$, that is, $S(f)=\left\{p \in M \mid \operatorname{rank} d f_{p}<2\right\}$. A map $f$ of $M$ into $\Sigma$ is said to be stable if there exists an open neighborhood of $f$ in $C^{\infty}(M, \Sigma)$ such that for any map $g$ in this neighborhood there exist diffeomorphisms $\Phi: M \rightarrow M$ and $\varphi: \Sigma \rightarrow \Sigma$ satisfying $g=\varphi \circ f \circ \Phi^{-1}$. Here $C^{\infty}(M, \Sigma)$ is the set of smooth maps of $M$ into $\Sigma$ with the Whitney $C^{\infty}$ topology. If $f$ is stable, there exist local coordinates centered at $p$ and $f(p)$ such that $f$ is locally described in one of the following way:

(1) $(u, x, y) \mapsto(u, x)$;

(2) $(u, x, y) \mapsto\left(u, x^{2}+y^{2}\right)$;

(3) $(u, x, y) \mapsto\left(u, x^{2}-y^{2}\right)$;

(4) $(u, x, y) \mapsto\left(u, y^{2}+u x-x^{3}\right)$.

(In the cases of (1), (2), (3) and (4), $p$ is called a regular point, a definite fold point, an indefinite fold point and a cusp point, respectively.) Further, we require that

(5) $f^{-1} \circ f(p) \cap S(f)=\{p\}$ for a cusp point $p$;

(6) restriction of $f$ to $S(f) \backslash\{$ cusp points $\}$ is an immersion with only normal crossings. 
Conversely, if a smooth map satisfies the above conditions, then it is a stable map. The stable maps form an open dense set in the space $C^{\infty}(M, \Sigma)$.

Let $M$ be a compact orientable 3-manifold with (possibly empty) boundary consisting of tori. A smooth map $f$ of $M$ into an orientable 2-manifold $\Sigma$ is called an $S$-map if

(1) the restriction of $f$ to Int $M$ is a stable map (here a stable map means that, as in the case where $M$ is closed, there exists an open neighborhood of $f$ in $C^{\infty}($ Int $M, \Sigma)$ such that for any map $g$ in this neighborhood there exist diffoemorphisms $\Phi: M \rightarrow M$ and $\varphi: \Sigma \rightarrow \Sigma$ satisfying $\left.g=\varphi \circ f \circ \Phi^{-1}\right)$;

(2) for each $p \in \partial M$ there exist a local coordinate $(u, x, y)$ centered at $p$, where $\partial M$ corresponds to $\{y=0\}$, and a local coordinate of $f(p)$ such that $f$ is locally described as $(u, x, y) \mapsto(u, x)$.

As in Saeki [36, we denote by $S_{0}(f), S_{1}(f)$ and $C(f)$ the sets of definite fold, indefinite fold, cusp points, respectively, of the restriction of $f$ to Int $M$.

Let $f$ be an $S$-map of a compact orientable 3 -manifold $M$ with (possibly empty) boundary consisting of tori into an orientable 2 -manifold $\Sigma$. We say that two points $p_{1}$ and $p_{2}$ are equivalent if they are contained in the same component of the fibers of $f$. We denote by $W_{f}$ the quotient space of $M$ with respect to the equivalence relation and by $q_{f}$ the quotient map. We define the map $\bar{f}: W_{f} \rightarrow N$ so that $f=\bar{f} \circ q_{f}$. The quotient space $W_{f}$, or the composition $\bar{f} \circ q_{f}$ is called the Stein factorization of $f$. The Stein factorization $W_{f}$ is homeomorphic to a polyhedron, that is, the underlying space of a finite 2-dimensional simplicial complex.

Let $f$ be an $S$-map of a compact orientable 3-manifold $M$ with (possibly empty) boundary consisting of tori into an orientable 2-manifold $\Sigma$. By Kushner-Levine-Porto [21] and Levine 24], the local models of the Stein factorization $W_{f}$ can be described as follows.

If $p \in S_{0}(f)\left(p \in C(f)\right.$, respectively), the projection $q_{f}(p) \mapsto f(p)$ of the Stein factorization looks locally as shown in Figure 5 (i) (Figure 5 (ii), respectively). In this case,

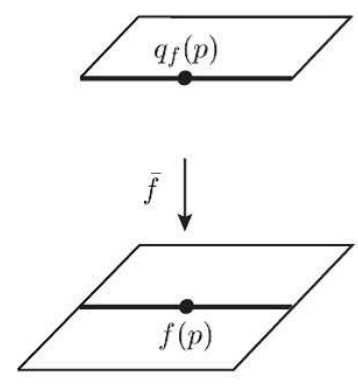

(i)

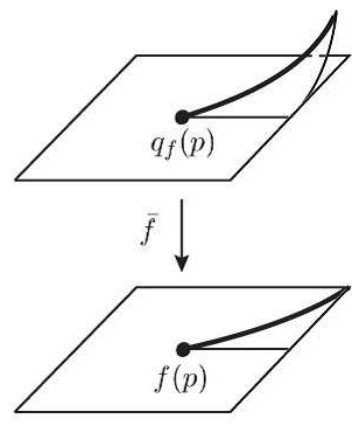

(ii)

Figure 5. The local model of the map $\bar{f}: W_{f} \rightarrow \Sigma$ for: (i) a definite fold point; (ii) a cusp point.

$q_{f}^{-1} \circ q_{f}(p)$ is called a singular fiber of type $\mathrm{I}^{0}$ (II ${ }^{a}$, respectively). See Saeki [37, Figure 3.4]. We remark that, in Saeki's book, these notations are defined for singular fibers of stable 
maps of orientable 4-manifolds into 3-manifolds. However, the classification of singular fibers of stable maps of orientable 3-manifolds into a plane coincide with that of singular fibers of codimension 0, 1, 2 of stable maps of orientable 4-manifolds into 3-manifolds as mentioned in [37, Remark 3.14]. For this reason, we consistently use the symbols in [37, Figure 3.4] also for the remaining types of singular fibers described below.

If $p \in S_{1}(f)$, the projection $q_{f}(p) \mapsto f(p)$ looks locally as one of the three models shown in Figure 6. Let $U_{1}, U_{2}$ and $U_{3}$ be regular neighborhoods of points $q_{f}(p)$ of types

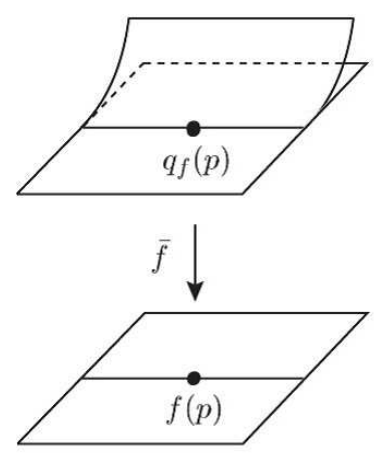

(i)

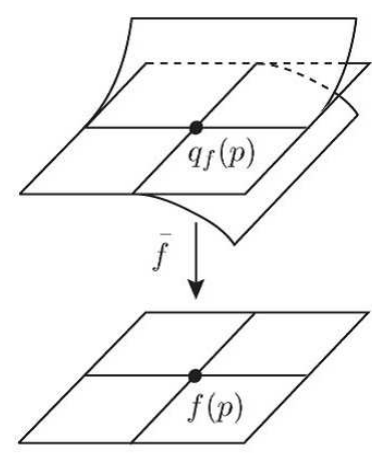

(ii)

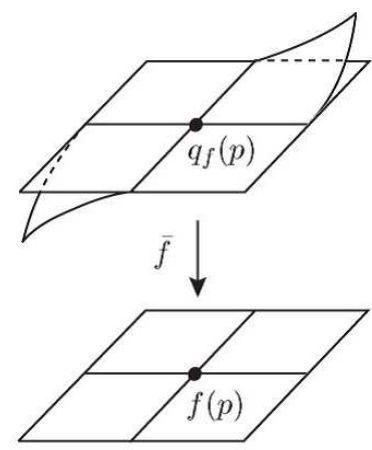

(iii)

Figure 6 . The three local models of the map $\bar{f}: W_{f} \rightarrow \Sigma$ for indefinite fold points.

(i), (ii) and (iii), respectively, shown in the figure. Then the preimage $q_{f}^{-1}\left(U_{1}\right) \subset M$ is diffeomorphic to $R \times[0,1]$, where $R$ is a pair of pants, and $f$ maps $q_{f}^{-1}\left(U_{1}\right)$ into $\Sigma$ as depicted in Figure 7. In this case $q_{f}^{-1} \circ q_{f}(p)$ is called a singular fiber of type $\mathrm{I}^{1}$. Also,
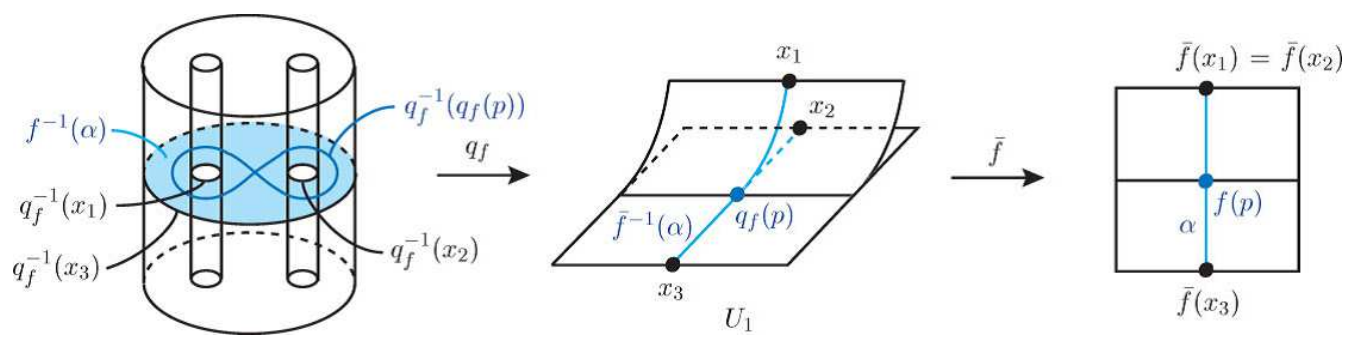

Figure 7. The Stein factorization of the restriction of $f$ to $f^{-1}\left(U_{1}\right)$. In this figure, $\alpha$ is a transverse arc for $f$ at $f(p)$. The singular fiber depicted in the figure is of Type $\mathrm{I}^{1}$.

the preimage $f^{-1}\left(U_{2}\right)\left(f^{-1}\left(U_{3}\right)\right.$, respectively) is diffeomorphic to $S \times[0,1]$, where $S$ is a disk with three holes, and $f$ maps $f^{-1}\left(U_{2}\right)\left(f^{-1}\left(U_{3}\right)\right.$, respectively) as drawn in Figure 8 (Figure 9, respectively). In this case $q_{f}^{-1} \circ q_{f}(p)$ is called a singular fiber of type $\mathrm{II}^{2}\left(\mathrm{II}^{3}\right.$, respectively). We call the image in $\Sigma$ of a singular fiber of type $\mathrm{II}^{2}$ or $\mathrm{II}^{3}$ a non-simple 


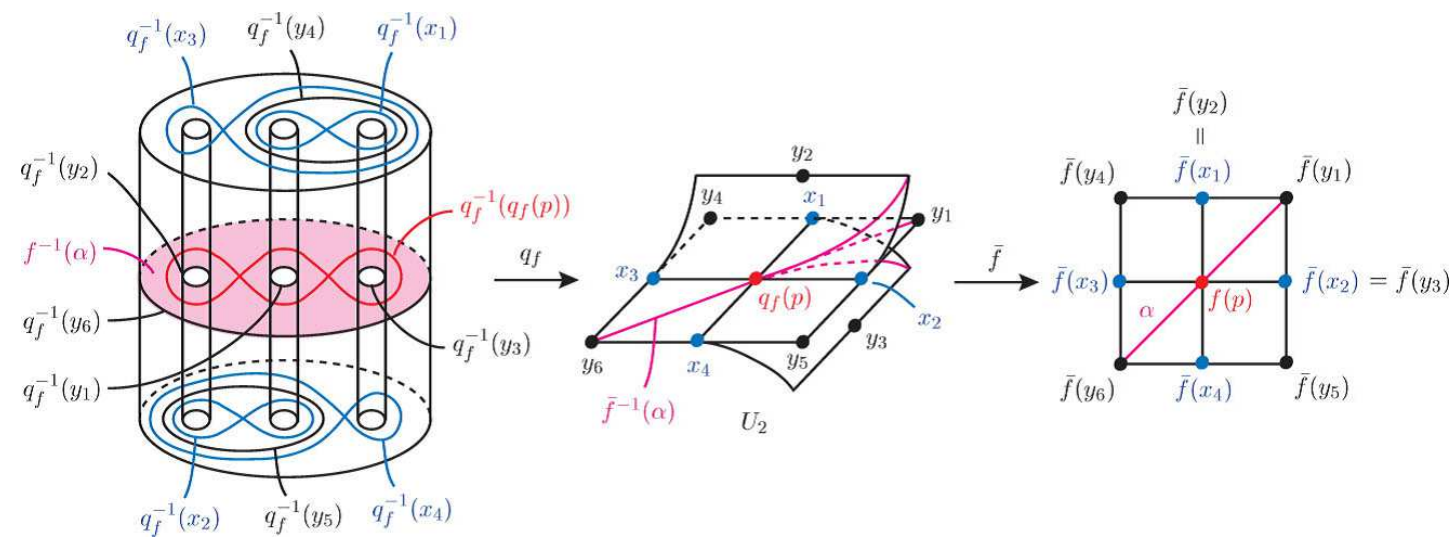

FiguRE 8. The Stein factorization of the restriction of $f$ to $f^{-1}\left(U_{2}\right)$. In this figure, $\alpha$ is a transverse arc for $f$ at $f(p)$. The singular fiber depicted in the figure is of Type $\mathrm{II}^{2}$.

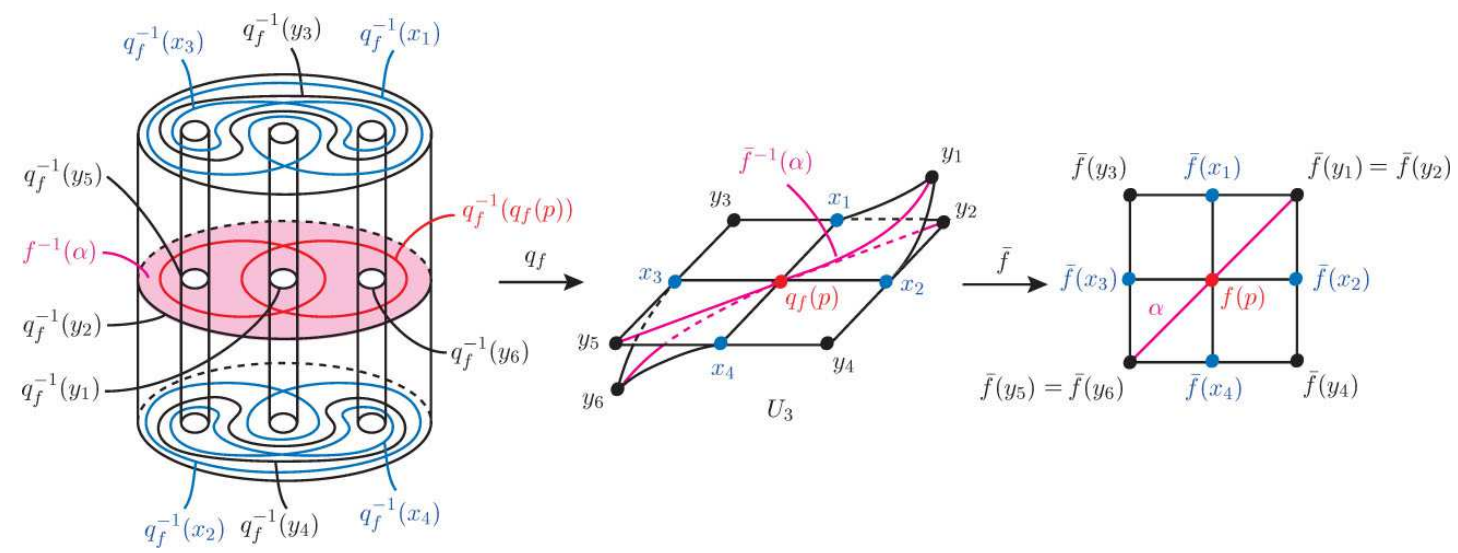

Figure 9. The Stein factorization of the restriction of $f$ to $f^{-1}\left(U_{3}\right)$. In this figure, $\alpha$ is a transverse arc for $f$ at $f(p)$. The singular fiber depicted in the figure is of Type $\mathrm{II}^{3}$.

crossing of $f$. We denote by $\mathrm{II}^{2}(f)$ and $\mathrm{II}^{3}(f)$ the sets of singular fibers of types $\mathrm{II}^{2}$ and $\mathrm{II}^{3}$, respectively, of $f$.

Definition. Let $M$ be a compact, orientable 3-manifold with (possibly empty) boundary consisting of tori and $L$ a (possibly empty) link in $M$. Let $f: M \rightarrow \Sigma$ be an S-map of $M$ into an orientable 2-manifold $\Sigma$. We say that $f$ is an $S$-map of $(M, L)$ (or simply of $L)$ if $S_{0}(f) \supset L$. An S-map $f$ of $(M, L)$ is said to be proper if $S_{0}(f)=L$. When $M$ is a closed 3-manifold, we call $f$ a stable map of $(M, L)$.

In the following, we give several examples that will be used later. All of them appeared essentially in Saeki [36]. 
Example 1. We identify $S^{2}$ with $\hat{\mathbb{C}}=\mathbb{C} \cup\{\infty\} . \quad D_{\alpha}=\{z \in \mathbb{C}|| z+1 / 2 \mid \leqslant 1 / 4\}$, $D_{\beta}=\{z \in \mathbb{C}|| z-1 / 2 \mid \leqslant 1 / 4\}, D_{\gamma}=\{z \in \mathbb{C}|| z \mid \geqslant 1\}, \alpha=\partial D_{\alpha}, \beta=\partial D_{\beta}, \gamma=\partial D_{\gamma}$.

Let $h: S^{2} \rightarrow \mathbb{R}$ be a height function such that

- $h(z)=h(-z)$ for all $z \in \mathbb{C}$;

- $h( \pm 1 / 2)=-1$ and $h(\infty)=1$

- $h(\alpha)=h(\beta)=-1 / 2$ and $h(\gamma)=1 / 2$; and

- $0 \in P$ is the unique critical point of index 1 .

For each integer $n$, let $\rho_{n}: S^{2} \times S^{1} \rightarrow[-1,1] \times S^{1}$ be the map defined by

$$
\rho_{n}(z, \theta)=(h(z \exp (\sqrt{-1}(-n \theta / 2))), \theta),
$$

where we identify $S^{1}$ with $\mathbb{Z} / 2 \pi \mathbb{Z}$. Let $\psi:[-1,1] \times S^{1} \rightarrow \mathbb{R}^{2}$ be an immersion.

(1) The map $f_{n}: S^{2} \times S^{1} \rightarrow \mathbb{R}^{2}$ defined by $f_{n}(p)=\psi \circ \rho_{n}(p)$ is a stable map without cusp points. For this stable map, the set $S_{1}\left(f_{n}\right)$ of indefinite fold points consists of a single circle, while the set $S_{0}\left(f_{n}\right)$ of definite fold points consists of three (two, respectively) circles when $n$ is an even (odd, respectively) integer.

(2) Let $f_{n}: S^{2} \times S^{1} \rightarrow \mathbb{R}^{2}$ be the stable map defined in (1). For each integer $n$, we set

$$
V_{n}=\left(S^{2} \times S^{1}\right) \backslash\left(\operatorname{Int}\left(D_{\gamma} \times S^{1}\right) \cup \operatorname{Int}\left\{(z \exp (\sqrt{-1}(n \theta / 2)), \theta) \mid z \in D_{\alpha} \cup D_{\beta}\right\}\right) .
$$

We note that $V_{n}$ can be identified with $\left(S^{2} \times S^{1}\right) \backslash \operatorname{Int} \operatorname{Nbd}\left(S_{0}\left(f_{n}\right)\right)$. In particular, when $n$ is an even integer, $V_{n}$ is diffeomorphic to $R \times S^{1}$, where $R$ is a pair of pants. The restriction of $\rho_{n}$ to $V_{n}$ is a map from $V_{n}$ to $[-1 / 2,1 / 2] \times S^{1}$. Then $\left.f_{n}\right|_{V_{n}}: V_{n} \rightarrow \mathbb{R}^{2}$ is an S-map without cusp or definite fold points while the set $S_{1}\left(\left.f_{n}\right|_{V_{n}}\right)$ of indefinite fold points consists of a single circle. This map will be used in the proof of Theorem 2.2.

(3) Set $A=\hat{\mathbb{C}} \backslash \operatorname{Int}\left(D_{\alpha} \cup D_{\beta}\right)$ and $\rho_{A}=\left.\rho_{0}\right|_{A \times S^{1}}$. The map $\psi \circ \rho_{A}: A \times S^{1} \rightarrow \mathbb{R}^{2}$ is an S-map without cusp points. Each of $S_{0}\left(\psi \circ \rho_{A}\right)$ and $S_{1}\left(\psi \circ \rho_{A}\right)$ consists of a single circle. This map will also be used in the proof of Theorem 2.2 .

(4) Set $D=\hat{\mathbb{C}} \backslash$ Int $D_{\gamma}$. The restriction of $\rho_{n}$ to $D \times S^{1}$ is a map from $D \times S^{1}$ to $[-1,1 / 2] \times S^{1}$. We identify $S^{3}$ with $\left(D \times S^{1}\right) \cup_{\varphi}\left(D^{2} \times S^{1}\right)$, where $\varphi: \partial D^{2} \times S^{1} \rightarrow$ $\partial D \times S^{1}$ is defined by $\varphi(\theta, \tau)=(\tau, \theta)$. Define the map $g_{n}: S^{3} \rightarrow \mathbb{R}^{2}$ by

$$
g_{n}(p)=\left\{\begin{array}{ll}
\psi_{1} \circ \rho_{n}(p) & \text { for } p \in D \times S^{1} \\
x & \text { for } p=(x, \theta) \in D^{2} \times S^{1}
\end{array},\right.
$$

where $\psi_{1}:[-1,1 / 2] \times S^{1} \hookrightarrow \mathbb{R}^{2}=\mathbb{C}$ is defined by $\psi_{1}(r, \theta)=(3 / 2-r) \exp (\sqrt{-1} \theta)$ and $D^{2}$ is identified with the unit disk on $\mathbb{R}^{2}$ centered at $(0,0)$. This is a stable map without cusp points. The set $S_{0}\left(g_{n}\right)$ of definite fold points is the $(2, n)$ torus link, in other words, $g_{n}$ is a proper stable map of the $(2, n)$-torus link. The Stein factorization $W_{g_{n}}$ of this map is shown in Figure 10, We note that the almost-special polyhedron $W_{g_{n}}$ equipped with the gleam $n / 2$ on its disk region is a (branched) shadow of the $(2, n)$-torus link. See Costantino-Thurston [12, Example 3.16].

Definition. Let $M$ be a compact, orientable 3-manifold with (possibly empty) boundary consisting of tori and $L$ a (possibly empty) link in $M$. Let $f:(M, L) \rightarrow \mathbb{R}^{2}$ be an S-map. 


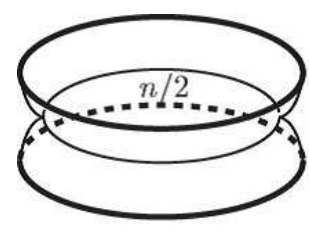

$n$ is even

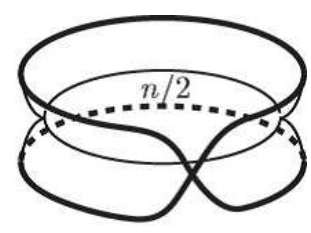

$n$ is odd

Figure 10. The Stein Factorization $W_{g_{n}}$.

The complexity of $f$, denoted by $c(f)$, is defined by $c(f)=\left|\mathrm{II}^{2}(f)\right|+2\left|\mathrm{II}^{3}(f)\right|$. The stable map complexity of $(M, L)$ (or simply of $L$ ), denoted by $\operatorname{smc}(M, L)$, is defined by $\operatorname{smc}(M, L)=\min _{f}\{c(f)\}$, where $f$ runs over all S-maps of $(M, L)$ into $\mathbb{R}^{2}$ without cusp points.

Example 2. In Example 1 (4), we have seen that the stable map complexity of a $(2, n)$ torus link is zero.

\section{BRANCHED SHADOW COMPLEXITY AND STABLE MAP COMPLEXITY}

Lemma 2.1. Let $M$ be a compact orientable 3-manifold with (possibly empty) boundary consisting of tori. Let $f: M \rightarrow S^{2}$ be an $S$-map. Then there exists an $S$-map $g: M \rightarrow \mathbb{R}^{2}$ satisfying $c(g)=\left|\mathrm{II}^{2}(f)\right|+2\left|\mathrm{II}^{3}(f)\right|$ and $S_{0}(f) \subset S_{0}(g)$.

Proof. The assertion follows from the construction of a stable map in Saeki [36, Lemma 3.6]. For completeness, we recall the argument.

Let $x \in S^{2}$ be a regular value of $f$. Choose two small disks $D_{0}$ and $D_{1}$ in $S^{2}$ so that $x \in D_{0} \subset \operatorname{Int} D_{1}$ and $f(S(f)) \cap D_{1}=\emptyset$.

Let $A$ and $\rho_{A}$ be as in Example1(3). Let $N_{1}, N_{2}, \cdots, N_{n}$ be the connected components of $f^{-1}\left(D_{1} \backslash\right.$ Int $\left.D_{0}\right)$. For each $1 \leqslant i \leqslant n$, there exist diffeomorphisms $t: N_{i} \rightarrow A \times S^{1}$ and $u:[-1 / 2,1] \times S^{1} \rightarrow D_{1} \backslash \operatorname{Int} D_{0}$ such that $u \circ \rho_{A} \circ t=\left.f\right|_{N_{i}}$.

Now we define the required map $g: M \rightarrow S^{2} \backslash\{x\} \cong \mathbb{R}^{2}$ by

$$
g(p)=\left\{\begin{array}{ll}
f(p) & \text { for } p \in f^{-1}\left(S^{2} \backslash \operatorname{Int} D_{1}\right) \\
u \circ \rho_{A} \circ t(p) & \text { for } p \in N_{i}, 1 \leqslant i \leqslant n \\
u_{0} \circ f(p) & \text { for } p \in f^{-1}\left(D_{0}\right),
\end{array} .\right.
$$

where $u_{0}: D_{0} \rightarrow S^{2} \backslash\{x\}$ is a smooth embedding of $D_{0}$ such that the restrictions of $u_{0} \circ f$ and $u \circ \rho_{A} \circ t$ to $f^{-1}\left(D_{0}\right) \cap N_{i}$ coincide. Since $\rho_{A}$ has no singular fibers of types $\mathrm{II}^{2}$ or $\mathrm{II}^{3}, g$ satisfies the requirement of the lemma.

Theorem 2.2. Let $M$ be a compact, orientable 3-manifold with (possibly empty) boundary consisting of tori and $L$ a (possibly empty) link in $M$. Then we have $\operatorname{bsc}(M, L)=$ $\operatorname{smc}(M, L)$.

Proof. We fix an orientation of $\mathbb{R}^{2}$.

We first prove the inequality $\operatorname{bsc}(M, L) \leqslant \operatorname{smc}(M, L)$. The argument essentially bases on the construction in the proof of Costantino-Thurston [12, Theorem 4.2]. Let $f: E(L) \rightarrow \mathbb{R}^{2}$ be an S-map without cusp points. Let $M \stackrel{q_{f}}{\longrightarrow} W_{f} \stackrel{\bar{f}}{\longrightarrow} \mathbb{R}^{2}$ be the Stein 
factorization of $f$. Then $Q=W_{f} \backslash \operatorname{Int} \operatorname{Nbd}\left(q_{f}\left(\mathrm{II}^{3}(f)\right) ; W_{f}\right)$ is an almost-special polyhedron. We note that the number of true vertices of $Q$ equals $\left|\mathrm{II}^{2}(f)\right|$. Put $N=q_{f}^{-1}(Q)$.

We note that $N \stackrel{q_{\left.f\right|_{N}}}{\longrightarrow} Q \stackrel{\left.\bar{f}\right|_{Q}}{\longrightarrow} \mathbb{R}^{2}$ is the Stein factorization of the map $\left.\bar{f}\right|_{Q} \circ q_{\left.f\right|_{N}}: N \rightarrow \mathbb{R}^{2}$. By [12], $Q$ is a shadow of $(N, L)$. We may equip an orientation on each region of $Q$ in such a way that $\left.\bar{f}\right|_{Q}$ is locally an orientation-preserving diffeomorphism at each interior point of the region. Apparently, these orientations of the regions of $Q$ give a branching $b_{0}$ of $Q$. We denote by $H_{1}, H_{2}, \ldots, H_{n}$ the components of $\partial Q \cap \partial \operatorname{Nbd}\left(q_{f}\left(\mathrm{II}^{3}(f)\right) ; W_{f}\right)$, each of which is a trivalent graph with 4 vertices. Let $N_{1}, N_{2}, \ldots, N_{\left|I^{3}(f)\right|}$ be the components of $q_{f}{ }^{-1} \operatorname{Nbd}\left(q_{f}\left(\mathrm{II}^{3}(f)\right) ; W_{f}\right)$, each of which is a genus 3 handlebody containing a singular fiber of type $\mathrm{II}^{3}$. By [12, the shadowed polyhedron $Q_{i}$ shown on the left-hand side in Figure 11 is a shadow of each $N_{i}$ and each component $H_{i}$ of $\partial Q$ can be capped off by $Q_{i}$ so that the resulting polyhedron $P$ is a shadow of $(M, L)$. We may equip a branching
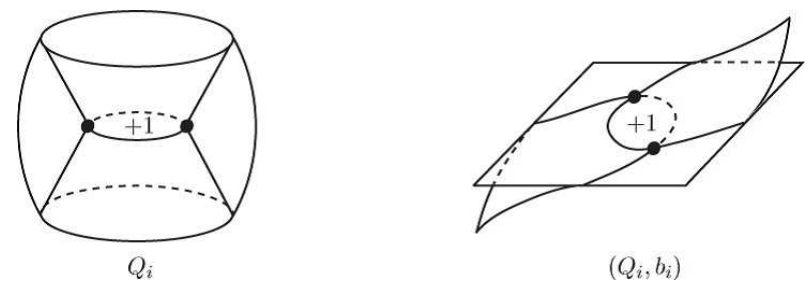

FiguRE 11. A branching of $Q_{i}$.

$b_{i}$ of $Q_{i}$ in such a way that the orientations on each edge of $H_{i}$ induced by $b_{0}$ and $b_{i}$ do not coincide, as shown on the right-hand side in Figure 11. Then the branchings $b_{0}$ and $b_{i}$ give a branching of $P$. It is straightforward from the above construction that the number of the true vertices of $P$ is $\left|\mathrm{II}^{2}(f)\right|+2\left|\mathrm{II}^{3}(f)\right|$ while $P$ has no boundary-vertices, so we are done.

In the following we will show the other inequality: $\operatorname{bsc}(M, L) \geqslant \operatorname{smc}(M, L)$. Let $P \subset W$ be a minimal branched shadow of $(M, L)$. By Lemma 2.1, it suffices to show that there exists an S-map $f: E(L) \rightarrow S^{2}$ satisfying $\left|\mathrm{II}^{2}(f)\right|+2\left|\mathrm{II}^{3}(f)\right|=\operatorname{bsc}(M, L)$. Suppose that $P$ contains non-empty boundary-vertices. Let $G_{1}, G_{2}, \ldots, G_{n}$ be the components of $\partial_{f} P$ containing at least one boundary vertex. For each $1 \leqslant i \leqslant n$, let $F_{i}$ be a compact orientable surface such that there exists an embedding $\iota_{i}: G_{i} \rightarrow \operatorname{Int} F_{i}$ and $F_{i}$ collapses onto $\iota_{i}\left(G_{i}\right)$. Fix an orientation of $F_{i}$ in an arbitrary way. We attach $P$ to each $F_{i}$ by the map $\iota_{i}$ and we denote the resulting polyhedron by $P^{*}$. The branching of $P$ and the orientations of $F_{1}, F_{2}, \ldots, F_{n}$ give a branching of $P^{*}$. See Figure 12, We note that by this operation, each boundary-vertex of $P$ gives rise to a true vertex of $P^{*}$ and no other true or boundary-vertices are produced. Thus $P^{*}$ has no boundary-vertices and we have $\left|V\left(P^{*}\right)\right|=c(P)=\operatorname{bsc}(M, L)$. Therefore we may assume without loss of generality that $P$ has no boundary-vertices.

We choose a collapsing $W \searrow P$ so that the induced projection $\pi: M \rightarrow P$ satisfies the following:

- for each $x \in \partial P, \pi^{-1}(p)$ is a single point; 


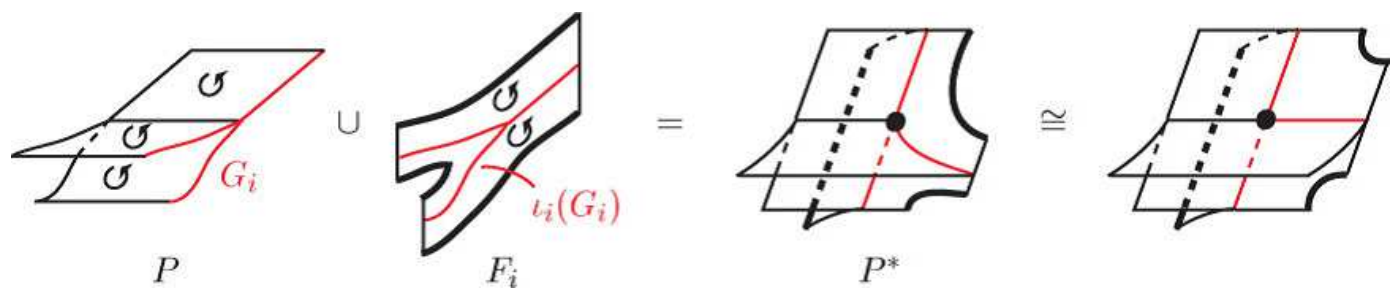

FiguRE 12. Elimination of boundary-vertices.

- for each $x \in V(P), \pi^{-1}(x) \cong T_{4}$, where $T_{4}$ is the suspension of four points;

- for each $x \in S(P) \backslash V(P), \pi^{-1}(x) \cong T_{3}$, where $T_{3}$ is the suspension of three points; and

- the restriction of $\pi$ to the preimage $\pi^{-1}(P \backslash(\partial P \cup S(P)))$ is a trivial, smooth $S^{1}$-bundle.

Note that for the construction of the projection $\pi$, we do not need a branching of $P$. For a point $x$ in $S(P) \backslash V(P)$, the preimage of $\operatorname{Nbd}(x)$ under $\pi$ is diffeomorphic to $R \times[0,1]$, where $R$ is a pair of pants, and $\pi$ is described as on the left-hand side in Figure 13 . Also,
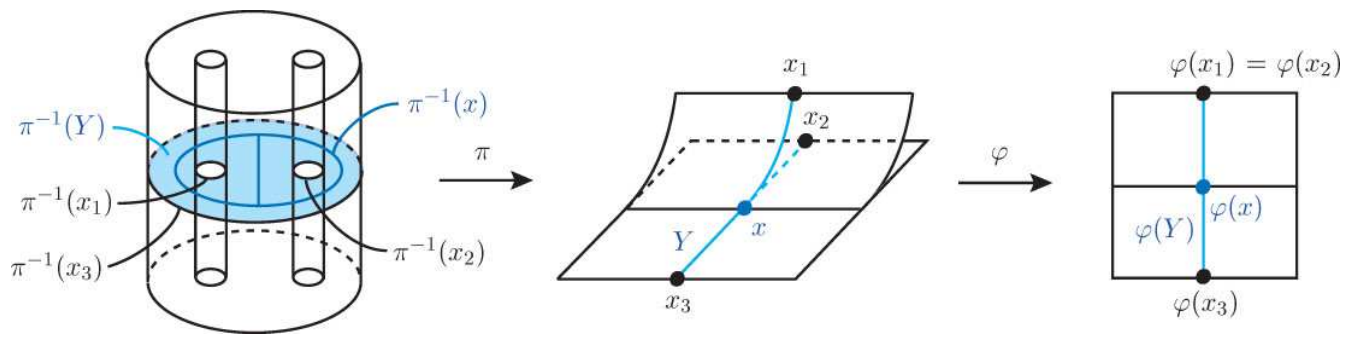

Figure 13. The local model of $\pi$ and $\varphi$ for $\operatorname{Nbd}(x ; P)$, where $x \in S(P) \backslash V(P)$.

for a point $x$ in $V(P)$, the preimage of $\operatorname{Nbd}(x)$ under $\pi$ is diffeomorphic to $S \times[0,1]$, where $S$ is a disk with 3 holes, and $\pi$ is described as on the left-hand side in Figure 14.

Let $v_{1}, v_{2}, \ldots, v_{n_{0}}$ be the true vertices of $P$. We put

$$
P^{\prime}=\overline{P \backslash\left(\operatorname{Nbd}(\partial P ; P) \cup\left(\bigcup_{i=1}^{n_{0}} \operatorname{Nbd}\left(v_{i} ; P\right)\right)\right)} \text {. }
$$

The set of components of $S\left(P^{\prime}\right)$ consists of intervals $e_{1}, e_{2}, \ldots, e_{m_{1}}$ and circles $l_{1}, l_{2}, \ldots, l_{n_{1}}$. We note that $\operatorname{Nbd}\left(e_{i} ; P^{\prime}\right)$ is homeomorphic to $Y \times[0,1]$, and $\operatorname{Nbd}\left(l_{j} ; P^{\prime}\right)$ is homeomorphic to $Y \times S^{1}$ or $Y \times[0,2 \pi] /(z, 2 \pi) \sim(\rho(z), 0)$, where $Y$ is a $Y$-shaped graph that we identify with $Y=\{r \exp (\sqrt{-1} \theta) \in \mathbb{C} \mid 0 \leqslant r \leqslant 1, \theta=0$ or $\pm 2 \pi / 3\}$ and $\rho: Y \rightarrow Y$ is defined by the complex conjugation. With this homeomorphisms, we equip each of $\operatorname{Nbd}\left(e_{i} ; P^{\prime}\right)$ and $\operatorname{Nbd}\left(l_{i} ; P^{\prime}\right)$ with a $Y$-bundle structure. In the following, we put the three boundary points of $Y$ as $a=\exp ((\sqrt{-1}) 2 / 3 \pi), b=\exp ((\sqrt{-1})(-2 / 3 \pi))$ and $c=1$. The set of 


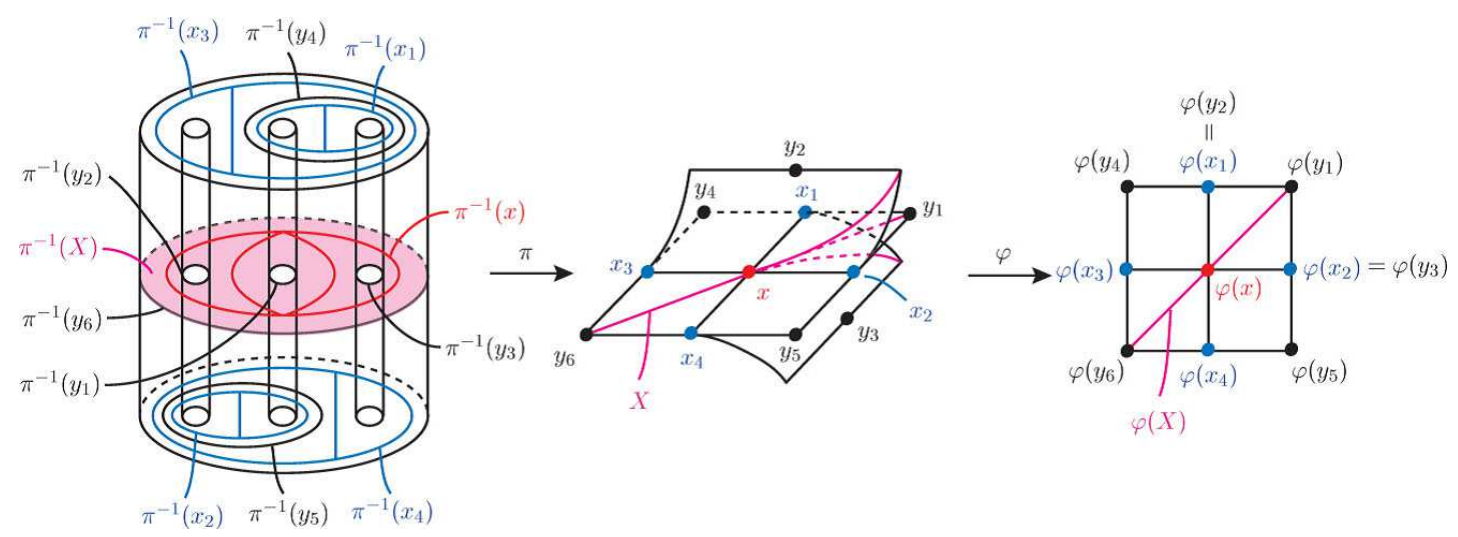

Figure 14. The local model of $\pi$ and $\varphi$ for $\operatorname{Nbd}(x ; P)$, where $x \in V(P)$.

components of

$$
\overline{P^{\prime} \backslash\left(\left(\bigcup_{i=1}^{m_{1}} \operatorname{Nbd}\left(e_{i} ; P^{\prime}\right)\right) \cup\left(\bigcup_{i=1}^{n_{1}} \operatorname{Nbd}\left(l_{i} ; P^{\prime}\right)\right)\right)}=P \backslash \operatorname{Int} \operatorname{Nbd}(\partial P \cup S(P) ; P)
$$

consists of oriented surfaces $R_{1}, R_{2}, \ldots, R_{n_{2}}$.

We set $N=\pi^{-1}(\operatorname{Nbd}(\partial P \cup S(P) ; P))$. Since $\operatorname{Nbd}(\partial P \cup S(P) ; P)$ is a branched polyhedron, there exists a map $\varphi: \operatorname{Nbd}(\partial P \cup S(P) ; P) \rightarrow S^{2}$ satisfying the following (see the right-hand side in Figures 13 and 14):

- $\varphi$ is generic on $S(P)$, that is, $\varphi$ is an injection on $S(P)$ except at finitely many double points, which are the projections of only two points of $S(P) \backslash V(P)$ and at the crossing points the two threads project to two different directions on $S^{2}$;

- For $1 \leqslant i<j \leqslant n_{0}, \varphi\left(\partial \operatorname{Nbd}\left(v_{i}\right)\right) \cong S^{1}$ and $\varphi\left(\operatorname{Nbd}\left(v_{i}\right)\right) \cap \varphi\left(\operatorname{Nbd}\left(v_{j}\right)\right)=\emptyset$;

- $\varphi$ is locally an orientation-preserving diffeomorphism at each point of $\operatorname{Nbd}(\partial P \cup$ $S(P) ; P) \backslash S(P)$;

- $\varphi(\operatorname{Nbd}(S(P) ; P))$ is a regular neighborhood of $\varphi(S(P))$ in $S^{2}$;

- for each $x \in e_{i}\left(x \in l_{i}\right.$, respectively), the fiber $Y_{x}$ of the $Y$-bundle structure of $\operatorname{Nbd}\left(e_{i} ; P^{\prime}\right)\left(\operatorname{Nbd}\left(l_{i} ; P^{\prime}\right)\right.$, respectively) is mapped into a segment by $r e^{i \theta} \mapsto r \cos \theta$.

Claim 1. There exists an $S$-map $g: N \rightarrow S^{2}$ with the Stein factorization $N \stackrel{q_{g}}{\longrightarrow} W_{g} \stackrel{\bar{g}}{\longrightarrow}$ $S^{2}$ such that

- $g(N)=\varphi \circ \pi(N)$;

- $W_{g} \cong \operatorname{Nbd}(\partial P \cup S(P) ; P)$; and

- the following diagram commutes:

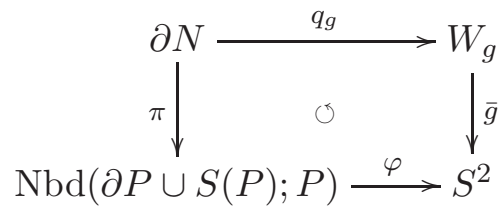


Proof of Claim 1. It is clear from the local model of the definite fold points (recall Figure 5 (i)) and the definition of $\varphi$ that $\pi^{-1}(\operatorname{Nbd}(\partial P ; P))$ consists of finitely many solid tori and there exists an S-map $g_{\partial P}: \pi^{-1}(\operatorname{Nbd}(\partial P ; P)) \rightarrow S^{2}$ with the Stein factorization $\pi^{-1}(\operatorname{Nbd}(\partial P ; P)) \stackrel{q_{g \partial P}}{\longrightarrow} W_{g_{\partial P}} \stackrel{\bar{g}_{\partial P}}{\longrightarrow} S^{2}$ such that

- $g_{\partial P}\left(\pi^{-1}(\operatorname{Nbd}(\partial P ; P))\right)=\varphi(\operatorname{Nbd}(\partial P ; P))$;

- $W_{g_{\partial P}} \cong \operatorname{Nbd}(\partial P ; P) ;$ and

- $\bar{g}_{\partial P} \circ q_{g_{\partial P}}=\varphi \circ \pi$ on $\partial \pi^{-1}(\operatorname{Nbd}(\partial P ; P))$.

For each $1 \leqslant i \leqslant n_{1}$, we identify $\operatorname{Nbd}\left(l_{i} ; P\right)$ with $Y \times S^{1}$ or $Y \times[0,2 \pi] /(z, 2 \pi) \sim(\rho(z), 0)$ as mentioned earlier. Here we identify them so that the points $(a, 0) \in Y \times S^{1}$ and $(b, 0) \in Y \times S^{1}$ lie on the regions of $P$ that induce the same orientation on $l_{i}$. The map $\left.\varphi\right|_{\operatorname{Nbd}\left(l_{i} ; P\right)}: \operatorname{Nbd}\left(l_{i} ; P\right) \rightarrow S^{2}$ factors through $\operatorname{Nbd}\left(l_{i} ; P\right) \stackrel{\varphi_{1}}{\longrightarrow}[-1 / 2,1 / 2] \times S^{1} \stackrel{\varphi_{2}}{\longrightarrow} S^{2}$, where $\varphi_{1}$ is defined by $\varphi_{1}(r \exp (\sqrt{-1}( \pm 2 \pi / 3)))=-r / 2$ and $\varphi_{1}(r)=r / 2$ for $0 \leqslant r \leqslant 1$. For $n \in \mathbb{Z}$, let $V_{n}$ and $\rho_{n}$ be as defined in Example 1 (2). Then by construction, we may identify $\pi^{-1}\left(Y \times S^{1}\right)$ with $V_{0}$ or $V_{1}$, depending on whether $\operatorname{Nbd}\left(l_{i} ; P\right)$ is $Y \times S^{1}$ or $Y \times[0,2 \pi] /(\rho(z), 2 \pi) \sim(z, 0)$, so that $\varphi_{2} \circ \rho_{n}$ coincides with $\left.\varphi \circ \pi\right|_{V_{n}}$ on $\partial V_{n}$. We denote the map $\varphi_{2} \circ \rho_{n}: \pi^{-1}\left(\operatorname{Nbd}\left(l_{i} ; P\right)\right) \rightarrow S^{2}$ by $g_{l_{i}}$.

For each $1 \leqslant j \leqslant m_{1}$, we identify $\operatorname{Nbd}\left(e_{j} ; P^{\prime}\right)$ with $Y \times[0,1]$. As in the above argument, we may assume that the points $(a, 0) \in Y \times S^{1}$ and $(b, 0) \in Y \times S^{1}$ lie on the regions of $P$ that induce the same orientation on $e_{j}$. The map $\left.\varphi\right|_{\mathrm{Nbd}\left(e_{j} ; P^{\prime}\right)}$ : $\operatorname{Nbd}\left(e_{j} ; P^{\prime}\right) \rightarrow S^{2}$ factors through $\operatorname{Nbd}\left(e_{j} ; P^{\prime}\right) \stackrel{\varphi_{1}}{\longrightarrow}[-1 / 2,1 / 2] \times[0,1] \stackrel{\varphi_{2}}{\longrightarrow} S^{2}$, where $\varphi_{1}$ is defined by $\varphi_{1}(r \exp (\sqrt{-1}( \pm 2 \pi / 3)))=-r / 2$ and $\varphi_{1}(r)=r / 2$ for $0 \leqslant r \leqslant 1$. Let $R=\hat{\mathbb{C}} \backslash \operatorname{Int}\left(D_{\alpha} \cup D_{\beta} \cup D_{\gamma}\right)$ and $\rho_{0}: R \times S^{1} \rightarrow[-1 / 2,1 / 2]$ be as in Example 1 (2). By construction, we may identify $\pi^{-1}(Y \times[0,1])$ with $R \times[0,1]$ so that $\left.\varphi_{2} \circ \rho_{0}\right|_{R \times[0,1]}$ coincides with $\left.\varphi \circ \pi\right|_{R \times[0,1]}$ on $\partial R \times[0,1]$. We denote the map $\left.\varphi_{2} \circ \rho_{0}\right|_{R \times[0,1]}: \pi^{-1}\left(\operatorname{Nbd}\left(e_{j} ; P^{\prime}\right)\right) \rightarrow S^{2}$ by $g_{e_{j}}$.

Finally, for each $1 \leqslant k \leqslant n_{0}$, we identify $\pi^{-1}\left(\operatorname{Nbd}\left(v_{k} ; P\right)\right)$ with $S \times[0,1]$ as shown in Figure 14, where we recall that $S$ is a disk with 3 holes. Let $g_{v_{k}}: S \times[0,1] \rightarrow S^{2}$ be the local model of an S-map in a small neighborhood of a singular fiber of type $\mathrm{II}^{2}$ shown in Figure 8 with the Stein factorization $S \times[0,1] \stackrel{q_{g_{v_{k}}}}{\longrightarrow} W_{g_{v_{k}}} \stackrel{\bar{g}_{v_{k}}}{\longrightarrow} S^{2}$ such that

- $\left.g_{v_{k}}\right|_{\partial S \times[0,1]}=\left.\varphi \circ \pi\right|_{\partial S \times[0,1]} ;$

- $\left.g_{v_{k}}(S \times\{t\})=\varphi \circ \pi(S \times\{t\})\right)$; and

- the Stein factorization $W_{g_{v_{k}}}$ is isomorphic to $\operatorname{Nbd}\left(v_{k} ; P\right)$ as branched polyhedron, where we equip an orientation of each region of $W_{g_{v_{k}}}$ in such a way that map $\bar{g}_{v_{k}}$ is locally an orientation-preserving diffeomorphism on the region.

Since $P$ is equipped with a branching, a small $C^{\infty}$-perturbation allows us to assume that, on each component of $\pi^{-1}\left(\operatorname{Nbd}\left(v_{k} ; P\right) \cap \operatorname{Nbd}\left(e_{j} ; P^{\prime}\right)\right)$, that is a pair of pants, $g_{v_{k}}$ and $g_{e_{j}}$ coincide.

Now, we define a map $g: N \rightarrow S^{2}$ by

$$
g(p)= \begin{cases}g_{\partial P(p)} & \text { for } p \in \pi^{-1}(\operatorname{Nbd}(\partial P ; P)) \\ g_{l_{i}}(p) & \text { for } p \in \pi^{-1}\left(\operatorname{Nbd}\left(l_{i} ; P^{\prime}\right)\right), 1 \leqslant i \leqslant n_{1} \\ g_{e_{j}}(p) & \text { for } p \in \pi^{-1}\left(\operatorname{Nbd}\left(e_{j} ; P^{\prime}\right)\right), 1 \leqslant j \leqslant m_{1} \\ g_{v_{k}}(p) & \text { for } p \in \pi^{-1}\left(\operatorname{Nbd}\left(v_{k} ; P\right)\right), 1 \leqslant k \leqslant n_{0}\end{cases}
$$


This completes the proof of the claim.

By the above claim, it suffices to show that, for each $1 \leqslant i \leqslant n_{2}$, there exists an S-map $g_{R_{i}}: \pi^{-1}\left(R_{i}\right) \rightarrow S^{2}$ such that

- $\left.g_{R_{i}}\right|_{\partial\left(\pi^{-1}\left(R_{i}\right)\right)}=\left.g\right|_{\partial\left(\pi^{-1}\left(R_{i}\right)\right)}$; and

- $g_{R_{i}}$ admits no singular fibers of types $\mathrm{II}^{2}$ or $\mathrm{II}^{3}$.

First we prove the assertion in the case where each surface $R_{i}$ is a disk for each $i \in\left\{1,2, \ldots, n_{2}\right\}$. We fix the index $i$ and prove the existence of such an S-map of $\pi^{-1}\left(R_{i}\right)$. Hereafter we identify $\pi^{-1}\left(R_{i}\right)$ with $R_{i} \times S^{1}$. By small perturbation of the map $g$, we may assume that $\bar{g}\left(\partial R_{i}\right)$ has only normal double points. Choose a smooth arc in $\bar{g}\left(\partial R_{i}\right)$ which starts and ends at the same point, i.e., a double point of $\bar{g}\left(\partial R_{i}\right)$, and has no other double points. Such a smooth arc can be found by choosing a suitable double point. Choose a band $b_{1}=[0,1] \times[0,1]$ in $R_{i}$ with $\operatorname{Nbd}(S(P) ; P) \cap b_{1}=\{0,1\} \times[0,1]$ and a map $\bar{g}_{b_{1}}: \operatorname{Nbd}(S(P) ; P) \cup b_{1} \rightarrow S^{2}$ such that $\left.\bar{g}_{b_{1}}\right|_{\operatorname{Nbd}(S(P) ; P)}=\bar{g}$ and the image $\bar{g}_{b_{1}}\left(b_{1}\right)$ of $b_{1}$ is as shown in Figure 15. This map induces an S-map $g_{b_{1}}: N \cup\left(b_{1} \times S^{1}\right) \rightarrow S^{2}$
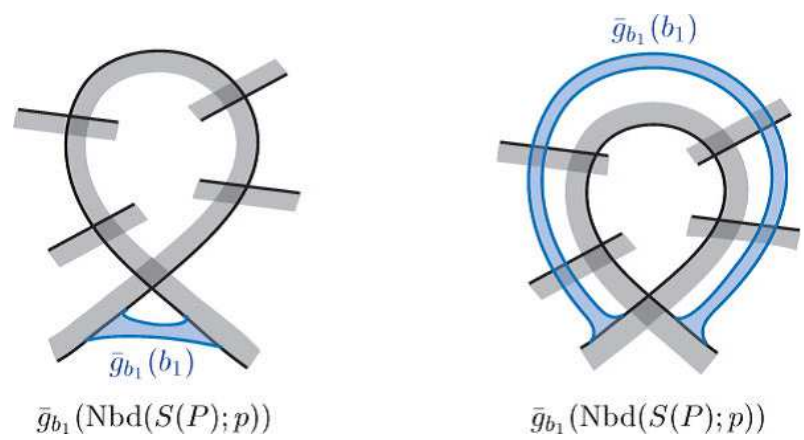

FiguRE 15. Extension of $\bar{g}$ over a band $b_{1}$.

satisfying

- $\left.g_{b_{1}}\right|_{N}=g$; and

- the Stein factorization of $g_{b_{1}}$ is given as $g_{b_{1}}=\bar{g}_{b_{1}} \circ q_{g_{b_{1}}}$, where $\left.q_{g_{b_{1}}}\right|_{b_{1} \times S^{1}}: b_{1} \times S^{1} \rightarrow$ $b_{1}$ is the first projection.

Note that $b_{1} \times S^{1} \subset R_{i} \times S^{1}$ and $b_{1} \times S^{1}$ is attached to $N$ according to the identification of $\pi^{-1}\left(R_{i}\right)$ with $R_{i} \times S^{1}$. Since $R_{i}$ is a disk, the closure of $R_{i} \backslash b_{1}$ consists of two disjoint disks, say $R_{i}^{\prime}$ and $R_{i}^{\prime \prime}$, such that

- $\bar{g}_{b_{1}}\left(\partial R_{i}^{\prime}\right)$ has just one double point; and

- the number of double points of $\bar{g}_{b_{1}}\left(\partial R_{i}^{\prime \prime}\right)$ is less than that of $\bar{g}\left(\partial R_{i}\right)$.

Next, we choose the surface $R_{i}^{\prime \prime}$ and apply the same argument, i.e., attach a band $b_{2}$ and make an S-map $g_{b_{2}}: N \cup\left(b_{1} \times S^{1}\right) \cup\left(b_{2} \times S^{1}\right) \rightarrow S^{2}$. We continue this process successively until $\bar{g}_{b_{j}}(c)$ has at most one double point for each boundary component $c$ of the closure of $R_{i} \backslash\left(b_{1} \cup \cdots \cup b_{j}\right)$. Since the number of double point of $\bar{g}\left(\partial R_{i}\right)$ is finite this process ends at a finite number of steps, say $n_{3}$ steps. As a consequence, we obtain an S-map $g_{B}: N \cup\left(B \times S^{1}\right) \rightarrow S^{2}$, where $B=\cup_{j=1}^{n_{3}} b_{j}$, such that 
- $\left.g_{B}\right|_{N}=g$;

- the Stein factorization of $g_{B}$ is given as $g_{B}=\bar{g}_{B} \circ q_{g_{B}}$, where $\bar{g}_{B}=\bar{g}_{b_{n_{3}}}$ and $\left.q_{g_{B}}\right|_{B \times S^{1}}: B \times S^{1} \rightarrow B$ is the first projection; and

- for each boundary component $c$ of the closure of $R_{i} \backslash B,\left.\bar{g}_{B}\right|_{c}$ is an immersion of a circle into $S^{2}$ such that it has at most one double point.

Suppose that $c_{0}$ is a boundary component of the closure of $R_{i} \backslash B$ such that $\bar{g}_{B}\left(c_{0}\right)$ has no double point and let $D_{c_{0}}$ denote the disk in $R_{i}$ bounded by $c_{0}$. In this case, the S-map $g_{c_{0}}: N \cup\left(B \times S^{1}\right) \cup\left(D_{c_{0}} \times S^{1}\right) \rightarrow S^{2}$ is easily obtained by choosing the image $\bar{g}_{c_{0}}\left(D_{c_{0}}\right)$ to be the disk bounded by $\bar{g}_{B}\left(c_{0}\right)$, where $N \cup\left(B \times S^{1}\right) \cup\left(D_{c_{0}} \times S^{1}\right) \subset M$ due to the identification of $\pi^{-1}\left(R_{i}\right)$ with $R_{i} \times S^{1}$.

Suppose that $c_{1}$ is a boundary component of the closure of $R_{i} \backslash B$ such that $\bar{g}_{B}\left(c_{1}\right)$ has exactly one double point. Let $V_{1}$ and $\left.f_{1}\right|_{V_{1}}: V_{1} \rightarrow \mathbb{R}^{2}$ be as in Example 1 (2). We regard $V_{1}$ as the exterior of an open tubular neighborhood of a $(2,1)$-cable knot in the solid torus $D^{2} \times S^{1}$ and set $T=\partial\left(D^{2} \times S^{1}\right)$ and $T_{1}=\partial V_{1} \backslash T$. Let $\phi: V_{1} \rightarrow V_{1}$ be an orientation-preserving diffeomorphism such that the positions of two boundary tori exchange, and set $f_{\phi}=\left.f_{1}\right|_{V_{1}} \circ \phi$. The image $f_{\phi}\left(V_{1}\right)$ is an annulus in $\mathbb{R}^{2}$. If the preimage of a point on the boundary of $f_{\phi}\left(V_{1}\right)$ is on $T$ then it is $\left\{a_{1}, a_{2}\right\} \times S^{1}$, where $a_{1}, a_{2}$ are distinct two points on $\partial D^{2}$. If the preimage is on $T_{1}$, then it is a longitude of the $(2,1)$-cable knot.

Consider the Stein factorization of $f_{\phi}$, i.e., $V_{1} \stackrel{q_{f_{\phi}}}{\longrightarrow} W_{f_{\phi}} \stackrel{\bar{f}_{\phi}}{\longrightarrow} S^{2}$. Attach $W_{f_{\phi}}$ to $\operatorname{Nbd}(S(P) ; P) \cup B$ smoothly so that $(\operatorname{Nbd}(S(P) ; P) \cup B) \cap W_{f_{\phi}}=q_{f_{\phi}}(T)=c_{1}$, and then attach $V_{1}$ to $N \cup\left(B \times S^{1}\right)$ so that $\left.q_{f_{\phi}}\right|_{T}=\left.g_{B}\right|_{T}$. Here the solid torus $D^{2} \times S^{1}$ including $V_{1}$ is identified with $\pi^{-1}\left(D_{c_{1}}\right) \subset \pi^{-1}\left(R_{i}\right)$, where $D_{c_{1}}$ is the disk in $R_{i}$ bounded by $c_{1}$. We need to modify the map $f_{\phi}$ to $f_{\phi}^{\prime}$, with keeping $q_{f_{\phi}}=q_{f_{\phi}^{\prime}}$, so that $\left.\bar{f}_{\phi}^{\prime}\right|_{q_{f_{\phi}}(T)}=\left.\bar{g}_{B}\right|_{c_{1}}$. In other words, modify it in such a way that the S-map $g_{c_{1}}: N \cup\left(B \times S^{1}\right) \cup V_{1} \rightarrow S^{2}$ defined by

$$
g_{c_{1}}= \begin{cases}g_{B}(p) & \text { for } p \in N \cup\left(B \times S^{1}\right) \\ f_{\phi}^{\prime}(p) & \text { for } p \in V_{1}\end{cases}
$$

is well-defined. The existence of such a modification of $f_{\phi}$ can be checked as follows: We set $N_{c_{1}}=\operatorname{Nbd}\left(c_{1} ; \operatorname{Nbd}(S(P) ; P) \cup B\right)$, which is an annulus. The image $\bar{g}_{B}\left(N_{c_{1}}\right)$ is an immersed annulus such that the image of one of the immersed boundary components is $\bar{g}_{B}\left(c_{1}\right)$. There are two regions in $S^{2} \backslash \operatorname{Int} \bar{g}_{B}\left(N_{c_{1}}\right)$ which have just one corner, and one of these regions is bounded by $\bar{g}_{B}\left(c_{1}\right)$. Taking the image of $f_{\phi}$ in this region, we see that $f_{\phi}$ can be modified so that it coincides with $\left.\bar{g}_{B}\right|_{c_{1}}$ on $q_{f_{\phi}}(T)$. See Figure 16 .

We attach a solid torus to $V_{1}$ along the boundary torus $T_{1}$ as follows: We first recall the construction of the map $h_{2}: T^{2} \times[0,1] \rightarrow \mathbb{R}^{2}$ given in Saeki [36, p. 680], where $T^{2}$ is a torus. Let $R$ be a pair of pants and $a$ be one of its boundary components. Let $\chi: \partial D^{2} \times S^{1} \rightarrow a \times S^{1}$ be the diffeomorphism defined by $\chi(\theta, \tau)=(\theta+\tau, \tau)$ and set $X=\left(R \times S^{1}\right) \cup_{\chi}\left(D^{2} \times S^{1}\right)$, which is diffeomorphic to $T^{2} \times[0,1]$. Then define $h_{2}: T^{2} \times[0,1] \rightarrow \mathbb{R}^{2}$ by

$$
h_{2}(p)= \begin{cases}\left(h\left(p_{1}\right), p_{2}\right) \in \mathbb{R} \times S^{1} \subset \mathbb{R}^{2} & \text { for } p=\left(p_{1}, p_{2}\right) \in R \times S^{1} \\ q_{1} \in D^{2} \subset \mathbb{R}^{2} & \text { for } p=\left(q_{1}, q_{2}\right) \in D^{2} \times S^{1},\end{cases}
$$




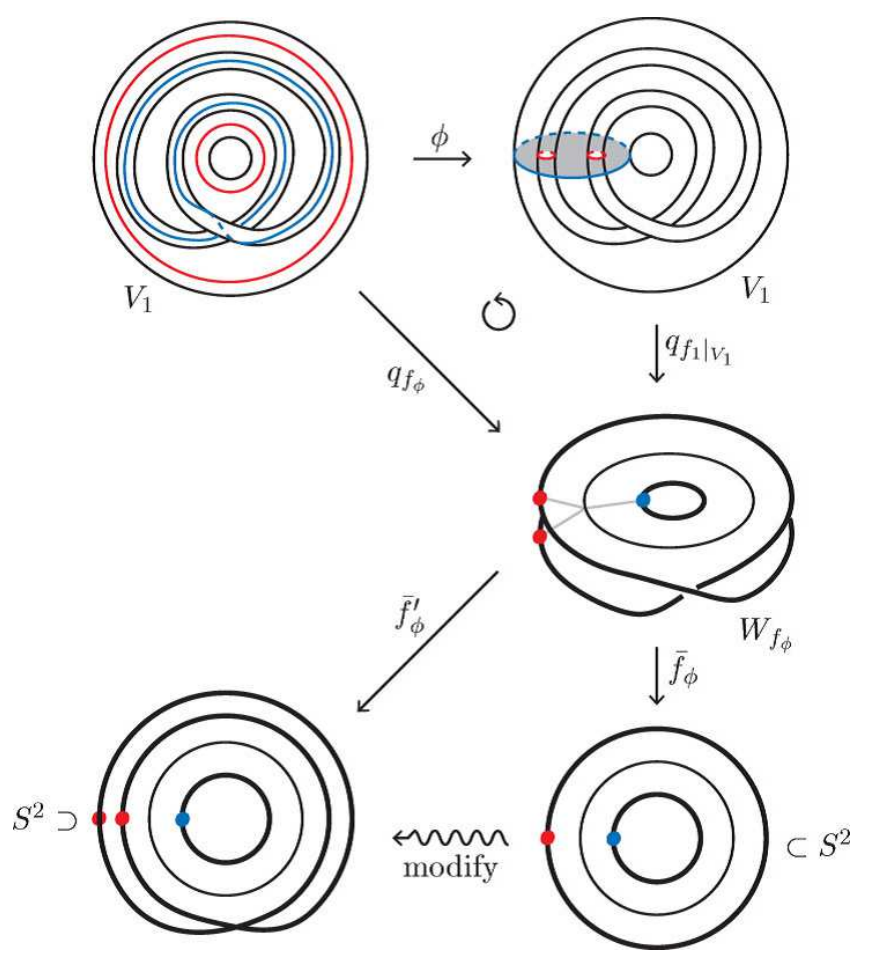

FIgURE 16. Extension of $g_{B}$ over $D_{c_{1}}$.

where $h: R \rightarrow \mathbb{R}$ is the Morse function as in Example 1 with regarding that $R$ is the pair of pants bounded by $\alpha, \beta$ and $\gamma$. The map $h_{2}$ has the following important property: For $i=0,1$, let $\mu_{i} \subset T^{2} \times\{i\}$ be a section of $h \times \operatorname{id}_{S^{1}}: R \times S^{1} \rightarrow \mathbb{R} \times S^{1}$ and $\lambda_{i}$ be a fiber of $h_{2}$ on $T^{2} \times\{i\}$. Then $h_{2}$ satisfies $\left[\lambda_{0}\right]=\left[\mu_{1}\right]$ and $\left[\mu_{0}\right]=\left[\lambda_{1}\right]$ in $H_{1}\left(T^{2} \times[0,1]\right)$.

Set $U=\operatorname{Nbd}\left(T_{1} ; V_{1}\right)$ and $T_{0}=\partial U \backslash T_{1}$. Let $D$ denote the disk in $S^{2}$ bounded by the simple closed curve $g_{c_{1}}\left(T_{0}\right)$. There exist diffeomorphisms $t: U \rightarrow T^{2} \times[0,1]$ and $u: D^{2} \rightarrow D \subset S^{2}$ such that $\left.u \circ h_{2} \circ t\right|_{T_{0}}=\left.g_{c_{1}}\right|_{T_{0}}$. Then we define an S-map $g_{c_{1}}^{\prime}: N \cup\left(B \times S^{1}\right) \cup V_{1} \rightarrow S^{2}$ by

$$
g_{c_{1}}^{\prime}= \begin{cases}g_{c_{1}}(p) & \text { for } p \in N \cup\left(B \times S^{1}\right) \cup V_{1} \backslash\left(\operatorname{Int} U \cup T_{1}\right) \\ u \circ h_{2} \circ t(p) & \text { for } p \in U .\end{cases}
$$

Since each fiber of the map $g_{c_{1}}^{\prime}$ on $T_{0}$ is a longitude, the fiber of $g_{c_{1}}^{\prime}$ on $T_{1}$ is the meridian of the $(2,1)$-cable knot. The compact tubular neighborhood of an $S^{1}$-family of definite fold points is a solid torus whose fiber on the boundary is the meridian. Attaching this solid torus to $N \cup\left(B \times S^{1}\right) \cup V_{1}$ along $T_{1}$, we obtain the S-map from $N \cup\left(B \times S^{1}\right) \cup\left(D^{2} \times S^{1}\right)$ to $S^{2}$, where $D^{2} \times S^{1}$ is the solid torus obtained from $V_{1}$ by filling the boundary $T_{1}$ canonically.

We apply the same construction for all boundary components of the closure of $R_{i} \backslash B$ and obtain an S-map $g_{C_{i}}: N \cup\left(R_{i} \times S^{1}\right) \rightarrow S^{2}$. 
Applying this construction to all surfaces $R_{1}, \ldots, R_{n_{2}}$, we obtain the S-map $g_{C}: M \rightarrow$ $S^{2}$ with the required properties. This completes the proof in the case where each surface $R_{i}$ is a disk.

Suppose that some of $R_{1}, \ldots, R_{n_{2}}$ is not a disk. Let $\Gamma$ be the union of mutually disjoint, essential simple closed curves in $R_{1} \cup \cdots \cup R_{n_{2}}$ that cuts $R_{1} \cup \cdots \cup R_{n_{2}}$ into planar surfaces each of which contains at most one boundary component of $\operatorname{Nbd}(\partial P \cup S(P) ; P)$. We remark that the polyhedron $P$ cut off by $\Gamma$ may not be connected. We denote by $\hat{P}$ the almost-special branched polyhedron obtained by cutting $P$ along $\Gamma$ and then capping off the new boundary components by disks. Let $\hat{M}$ be the 3 -manifold represented by $\hat{P}$ after giving suitable gleams to the new disk regions. Since each region of $\hat{P}$ is a disk, by applying the above argument, we obtain an S-map $g_{C}: \hat{M} \rightarrow S^{2}$. To recover $P$, we first remove suitable number of disks from the regions of $\hat{P}$ and also remove their preimages in $\hat{M}$. We assume that the removed disks are near the boundary of $\operatorname{Nbd}(S(P) ; P)$ and it is sufficiently small so that their images in $S^{2}$ have no intersection.

Let $D_{0}$ and $D_{1}$ be disks in $\hat{P}$ whose boundaries will be identified. Set $A_{j}=\operatorname{Nbd}\left(\partial D_{j} ; \hat{P} \backslash\right.$ Int $\left.D_{j}\right)$ for $j=0,1$. The preimage $q_{g_{C}}^{-1}\left(A_{j}\right)$ has two boundary components, one of which is $q_{g_{C}}^{-1}\left(\partial D_{j}\right)$ named $T_{j, 1}$. We denote the other boundary component by $T_{j, 0}$. Let $A$ and $\rho_{0}$ be as in Example1 (3). For each $j=0,1$, there exist diffeomorphisms $t_{j}: q_{g_{C}}^{-1}\left(A_{j}\right) \rightarrow A \times S^{1}$ and $u_{j}:[-1 / 2,1] \times S^{1} \rightarrow \bar{g}_{C}\left(A_{j}\right)$ such that $\left.u_{j} \circ \rho_{0} \circ t_{j}\right|_{T_{j, 0}}=\left.g_{C}\right|_{T_{j, 0}}$. Then we define an S-map $g_{D}: \hat{M} \backslash q_{g_{C}}^{-1}\left(\operatorname{Int}\left(D_{0} \cup D_{1}\right)\right) \rightarrow S^{2}$ by

$$
g_{D}(p)= \begin{cases}g_{C}(p) & \text { for } p \notin q_{g_{C}}^{-1}\left(A_{j} \cup D_{j}\right) \\ u_{j} \circ \rho_{0} \circ t_{j}(p) & \text { for } p \in q_{g_{C}}^{-1}\left(A_{j}\right) .\end{cases}
$$

Remark that $g_{D}\left(T_{0,1}\right)$ and $g_{D}\left(T_{1,1}\right)$ are simple closed curves in $S^{2}$ and the interior of the annulus bounded by these curves does not intersect $g_{D}\left(A_{j}\right)$ for $j=0,1$. We attach an annulus $A_{2}$ between $\partial D_{0}$ and $\partial D_{1}$ and define the S-map $g_{D}^{\prime}: M^{\prime} \rightarrow S^{2}$ with Stein factorization $M^{\prime} \stackrel{q_{g_{D}^{\prime}}}{\longrightarrow} W_{g_{D}^{\prime}} \stackrel{\bar{g}_{D}^{\prime}}{\longrightarrow} S^{2}$ such that $\bar{g}_{D}^{\prime}\left(A_{2}\right)$ is the annulus bounded by $g_{D}\left(T_{0,1}\right)$ and $g_{D}\left(T_{1,1}\right)$, where $M^{\prime}=\left(\hat{M} \backslash q_{g_{C}}^{-1}\left(\operatorname{Int}\left(D_{0} \cup D_{1}\right)\right)\right) \cup\left(A_{2} \times S^{1}\right)$. Applying this construction for each pair of disks in $\hat{P}$ whose boundary should be identified, and adjusting the gleam by removing and re-gluing a compact neighborhood of the fiber of a point in $\partial R_{i}$ with keeping the existence of an S-map, we obtain the S-map $g_{E}: M \rightarrow S^{2}$ with the required properties. This completes the proof.

Remark 2.3. (1) Let $(M, L)$ be as in Theorem 2.2, and let $f:(M, L) \rightarrow \mathbb{R}^{2}$ be an S-map. If $\mathrm{II}^{3}(f)=\emptyset$, the Stein factorization $W_{f}$ is exactly a branched shadow of $(M, L)$. Suppose that $\mathrm{II}^{3}(f) \neq \emptyset$, and let $N_{1}, N_{2}, \ldots, N_{n}$ be closed neighborhoods of the singular fibers of type $\mathrm{II}^{3}$. We recall that each $N_{i}$ is a genus 3 handlebody. By the latter part of the proof of Theorem 2.2, we may construct a map $g_{i}: N_{i} \rightarrow$ $\mathbb{R}^{2}$ using the shadowed branched polyhedron depicted in Figure 11, Exchanging $f$ with $g_{i}$ inside $N_{i}$ for each $i \in\{1,2, \ldots, n\}$, we get a new S-map $(M, L) \rightarrow \mathbb{R}^{2}$ having no singular fibers of type $\mathrm{II}^{3}$.

(2) Let $(M, L)$ be as in Theorem 2.2 and let $P$ be its branched shadow having no boundary-vertices. By the latter part of the proof of Theorem 2.2, we may 
construct an S-map $f: E(L) \rightarrow \mathbb{R}^{2}$ with $\left|\mathrm{II}^{2}(f)\right|=c(P)$ and $\mathrm{II}^{3}(f)=\emptyset$. Then the Stein factorization $W_{f}$ is also an almost-special polyhedron and we have a natural inclusion $S(P) \subset S\left(W_{f}\right)$. Apparently, each "region" of $W_{f}$ is a planar surface. However, even if all the regions of $P$ are planar surfaces, $W_{f}$ is not homeomorphic to $P$ in general.

(3) In 20], the second-named author showed that the Heegaard genus of a closed orientable 3-manifold coincides with an invariant defined by branched spines. (See Endoh-Ishii [13] for details of the invariant.) Here, a spine of a closed 3-manifold $M$ is an almost-special polyhedron $P$ embedded in $M$ so that $M$ collapsed onto $P$ after removing a small open ball from $M$. In other words, branched spines determine the minimal number of the critical points of Morse functions on $M$. Theorem 2.2 can be compared with this fact.

The following is a straightforward consequence of Theorem 2.2 (The case where $M$ is closed and $L=\emptyset$ is proved by Kalmár-Stipsicz [18, Theorem 1.4]). See also (1) of the above remark.

Corollary 2.4. Let $M$ be a compact, orientable 3-manifold with (possibly empty) boundary consisting of tori and $L$ a (possibly empty) link in $M$. Then there exists an $S$-map $f:(M, L) \rightarrow \mathbb{R}^{2}$ without cusp points satisfying $\left|\mathrm{II}^{2}(f)\right|=\operatorname{smc}(M, L)$ and $\mathrm{II}^{3}(f)=\emptyset$.

Lemma 2.5. Let $M_{1}$ and $M_{2}$ be compact orientable 3 -manifolds. Then $\operatorname{bsc}\left(M_{1} \# M_{2}\right) \leqslant$ $\operatorname{bsc}\left(M_{1}\right)+\operatorname{bsc}\left(M_{2}\right)$.

Proof. Let $P_{1}$ and $P_{2}$ be branched shadows of $M_{1}$ and $M_{2}$, respectively. Choose an interior point $p_{i}$ of a region of $P_{i}$ for each $i \in\{1,2\}$. By Costantino-Thurston [12, Lemma 3.2], the polyhedron $P$ obtained by gluing small regular neighborhoods of $p_{1}$ and $p_{2}$ is a shadow of $M$ (after putting the gleam 0 on the new disk region.) The polyhedron $P$ apparently admits a branching.

By Theorem 2.2 and Lemma 2.5, the following holds.

Corollary 2.6. Let $M_{1}$ and $M_{2}$ be compact orientable 3-manifolds with (possibly empty) boundary consisting of tori. Then we have $\operatorname{smc}\left(M_{1} \# M_{2}\right) \leqslant \operatorname{smc}\left(M_{1}\right)+\operatorname{smc}\left(M_{2}\right)$.

Lemma 2.7. (1) Let $M_{1}$ and $M_{2}$ be compact orientable 3-manifolds such that both of $\partial M_{1}$ and $\partial M_{2}$ contain torus components $T_{1}$ and $T_{2}$, respectively. Let $\varphi: T_{1} \rightarrow T_{2}$ be a diffeomorphism. Then $\operatorname{bsc}\left(M_{1} \cup_{\varphi} M_{2}\right) \leqslant \operatorname{bsc}\left(M_{1}\right)+\operatorname{bsc}\left(M_{2}\right)$.

(2) Let $M$ be a compact oriented 3-manifold such that $\partial M$ contains at least two torus components $T_{1}$ and $T_{2}$, which are equipped with the orientations induced from that of $M$. Let $\varphi: T_{1} \rightarrow T_{2}$ be an orientation-reversing diffeomorphism. Then $\operatorname{bsc}(M / \varphi) \leqslant \operatorname{bsc}(M)$.

Proof. (11) Let $P_{1}$ and $P_{2}$ be branched shadows of $M_{1}$ and $M_{2}$, respectively. Let $l_{1}$ and $l_{2}$ be the boundary circles of $P_{1}$ and $P_{2}$ that correspond to the boundary tori $T_{1}$ and $T_{2}$, respectively. By Costantino-Thurston [12, Proposition 3.27], a shadow $P$ of $M_{1} \cup \varphi M_{2}$ is obtained by

$$
P_{1} \cup_{l_{1}=\alpha_{1}} Q \cup_{l_{2}=\alpha_{2}} P_{2},
$$



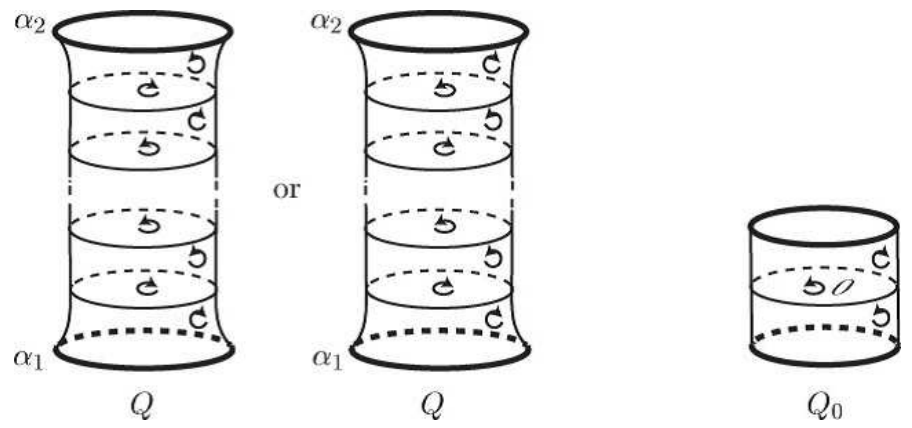

FiguRE 17. Branched shadows in the thickened torus.

where $Q$ is one of the two oriented polyhedra shown in Figure 17 equipped with certain gleams. Then the orientations of the regions of $Q$ shown in the figure extends to the whole of $P$, after reversing the orientations of the regions of $P_{1}$ or $P_{2}$, if necessary.

(2) Let $P_{1}$ be a branched shadow of $M$. Let $l_{1}$ and $l_{2}$ be the boundary circles of $P_{1}$ that correspond to the boundary tori $T_{1}$ and $T_{2}$, respectively. As in (11), the polyhedron $P$ obtained by attaching $Q$ along the loops $l_{1}$ and $l_{2}$ is a shadow of $\operatorname{bsc}(M / \varphi)$. If the orientations of the regions of $Q$ shown in the figure extends to the whole of $P$ we are done. Otherwise, we first attach a shadow polyhedron $Q_{0}$ shown on the right-hand side in Figure 17, to $Q$ along $\alpha_{1}$ and then we apply the former operation using $Q_{0} \cup Q$ instead of $Q$. This does not affect the topology of the resulting manifold, and now we may extend the orientation of the regions of $Q_{0} \cup Q$ to the whole of $P$.

Theorem 2.2 and Lemma 2.7 yield the following:

Corollary 2.8. (1) Let $M_{1}$ and $M_{2}$ be compact orientable 3-manifolds with boundary consisting of tori. Choose a component $T_{i}$ of $\partial M_{i}$ for each $i \in\{1,2\}$. Let $\varphi: T_{1} \rightarrow T_{2}$ be a diffeomorphism. Then $\operatorname{smc}\left(M_{1} \cup_{\varphi} M_{2}\right) \leqslant \operatorname{smc}\left(M_{1}\right)+\operatorname{smc}\left(M_{2}\right)$.

(2) Let $M$ be a compact oriented 3-manifold with boundary consisting of at least two tori. Choose two components $T_{1}$ and $T_{2}$ of $\partial M$, and equip them with the orientations induced from that of $M$. Let $\varphi: T_{1} \rightarrow T_{2}$ be an orientation-reversing diffeomorphism. Then $\operatorname{smc}(M / \varphi) \leqslant \operatorname{smc}(M)$.

For a link $L$ in a 3 -manifold $M$, we denote by $E(L)$ its exterior, that is, the complement of an open tubular neighborhood of $L$.

Lemma 2.9. Let $M$ be a compact orientable 3-manifold with (possibly empty) boundary consisting of tori, and let $L$ be a link in $M$. Then we have $\operatorname{bsc}(M) \leqslant \operatorname{bsc}(M, L)=$ $\operatorname{bsc}(E(L))$.

Proof. The left equality follows immediately from the definition.

We recall that a disk equipped with the gleam 0 is a branched shadow of $D^{2} \times S^{1}$. Hence by Lemma 2.7 we have

$$
\operatorname{bsc}(M, L) \leqslant \operatorname{bsc}(E(L))+\operatorname{bsc}\left(D^{2} \times S^{1}\right)=\operatorname{bsc}(E(L)) .
$$


The other inequality follows from the fact that by changing the colors of the boundary circles of a given branched shadow of $(M, L)$ corresponding to $L$, which are all " $i$ ", to "e"'s, we get a branched shadow of $E(L)$.

By Theorem 2.2 and Lemma 2.9 we have the following:

Corollary 2.10. Let $M$ be a compact, orientable 3-manifold with (possibly empty) boundary consisting of tori and $L$ a ink in $M$. Then we have $\operatorname{smc}(M) \leqslant \operatorname{smc}(M, L)=$ $\operatorname{smc}(E(L))$.

We recall that a compact orientable 3-manifold is called a graph manifold if we can cut it off by embedded tori into $S^{1}$-bundles over surfaces. A (possibly empty) link in a compact orientable 3-manifold is called a graph link if its exterior is a graph manifold.

Proposition 2.11. Let $M$ be a compact, orientable 3-manifold with (possibly empty) boundary consisting of tori and $L$ a (possibly empty) link in $M$. Then the following conditions are equivalent:

(1) $\operatorname{sc}(M, L)=0$

(2) $\operatorname{bsc}(M, L)=0$

(3) $L$ is a graph link.

Proof. By definition, (11) follows from (2). Also, by the proof of Costantino-Thurston [12, Proposition 3.31], (3) follows from (11). Below we show that (2) follows from (3). Suppose that $L$ is a graph (possibly empty) link. By Lemma 2.9, it suffices to show that $\operatorname{bsc}(E(L))=0$. By Saeki [36], there exists an S-map $f: E(L) \rightarrow S^{2}$ such that $\left.f\right|_{S(f)}$ is a smooth embedding. In particular, $f$ has no singular fibers of types $\mathrm{II}^{2}$ or $\mathrm{II}^{3}$, and hence $\operatorname{smc}(E(L))=0$. Thus by Corollary 2.10 we have $\operatorname{smc}(M, L)=0$. Now we get $\operatorname{bsc}(M, L)=0$ by Theorem 2.2 .

\section{STABLE MAPS OF LINKS}

In this section we will always identify $S^{1}$ with $\mathbb{R} / 2 \pi \mathbb{Z}$.

Let $D$ be a flat oriented disk properly embedded in the standard 4-ball $B^{4}$, and let $D^{\prime}$ be the closure of $D \backslash \operatorname{Nbd}(\partial D ; D)$. Let $\pi: S^{3} \rightarrow D$ be the projection induced by a collapsing $B^{4} \searrow D$. We may assume without loss of generality that the preimage $V_{h}=\pi^{-1}\left(D^{\prime}\right)$ is a solid torus, that we identify with $D^{\prime} \times S^{1}$, and $\left.\pi\right|_{V_{h}}$ is an S-map without singular points. We put $V_{v}=S^{3} \backslash \operatorname{Int} V_{h}$. Let $L=L_{1} \sqcup L_{2} \sqcup \cdots \sqcup L_{n}$ be an $n$-component link in $S^{3}$. We can push $L$ by isotopy so that $L$ lies in $D^{\prime} \times[[-\varepsilon],[+\varepsilon]]$, where $\varepsilon$ is a sufficiently small positive real number, and $\left.\pi\right|_{V_{h}}$ is generic with respect to $L$. The image $\pi(L)$, together with over/under crossing information at each double point, gives a diagram $D_{L}$ for $L$ on $D^{\prime}$. Consider the mapping cylinder

$$
P_{D_{L}}^{*}=\left((L \times[0,1]) \sqcup D^{\prime}\right) /(x, 0) \sim \pi(x) .
$$

Then $P_{D_{L}}^{*}$, together with a color " $i$ " of each $L_{j} \times\{1\} \subset \partial P_{L}^{*} \mathrm{C}$ is a shadow of $\left(S^{3}, L\right)$. Refer to Costantino-Thurston [12, Examples 3.15-17]. We fix an orientation of each component of $L$. The set of the regions of $P_{D_{L}}^{*}$ consists of subsurfaces of $D^{\prime}$ and $A_{j}=\left(L_{j} \times[0,1]\right) / \sim$, $j \in\{1,2, \ldots, n\}$. To these subsurfaces of $D^{\prime}$, we give the orientations induced by the prefixed orientation of $D^{\prime}$. Also, we give an orientation to each $A_{j}$ in such a way that 
the induced orientation of $L_{j} \times\{1\}$ coincides with that of the prefixed orientation of $L_{j}$. See Figure 18. With these orientations of the regions, $P_{D_{L}}^{*}$ becomes a branched shadow
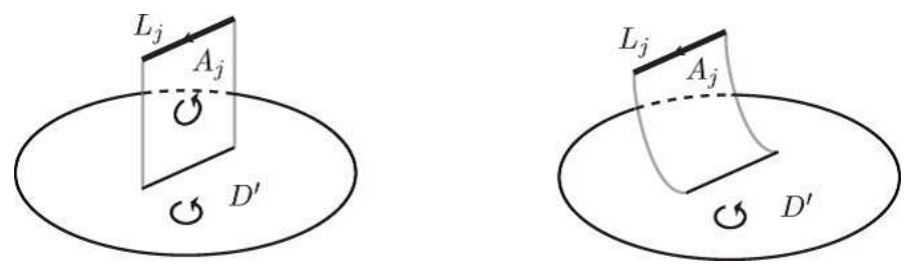

Figure 18. The branching on $A_{j}$.

of $\left(S^{3}, L\right)$. Let $R$ be the region of $P_{D_{L}}^{*}$ touching $\partial D^{\prime}$.

We say that the oriented link diagram $D_{L}$ is admissible if $R$ satisfies the following:

- the closure of $R$ is diffeomorphic to an annulus; and

- on each component of $S\left(P_{D_{L}}^{*}\right) \backslash V\left(P_{D_{L}}^{*}\right)$ touched by $R$ and $A_{j}(j \in\{1,2, \ldots, n\})$, these two regions induce the same orientation.

We note that if we choose, for example, $D_{L}$ to be a closed braid presentation of $L$ and give each component of $L$ a suitable orientation, then $R$ satisfies these conditions. If $D_{L}$ is admissible, $P_{D_{L}}=P_{D_{L}}^{*} \backslash R$ is still a branched shadow of $\left(S^{3}, L\right)$. In the following we will discuss this branched shadow.

Let $f:\left(S^{3}, L\right) \rightarrow \mathbb{R}^{2}$ be a stable map constructed from $P_{L}$ by the argument in Theorem 2.2. Let $S^{3} \stackrel{q_{f}}{\longrightarrow} W_{f} \stackrel{\bar{f}}{\longrightarrow} S^{2}$ be the Stein factorization of $f$. From the construction of the stable map, we can identify the quotient space $W_{f}$ with $P_{D_{L}}$ in a natural way. The preimage of a point of $P_{D_{L}}$ under the map $q_{f}$ can be described as follows:

(1) Let $x_{1}$ be a point in $D^{\prime} \backslash P_{D_{L}}$. Then the preimage $q_{f}^{-1}\left(x_{1}\right)$, which is a regular fiber of $f$, is $\left\{x_{1}\right\} \times S^{1} \subset V_{h}$. See Figure 19,

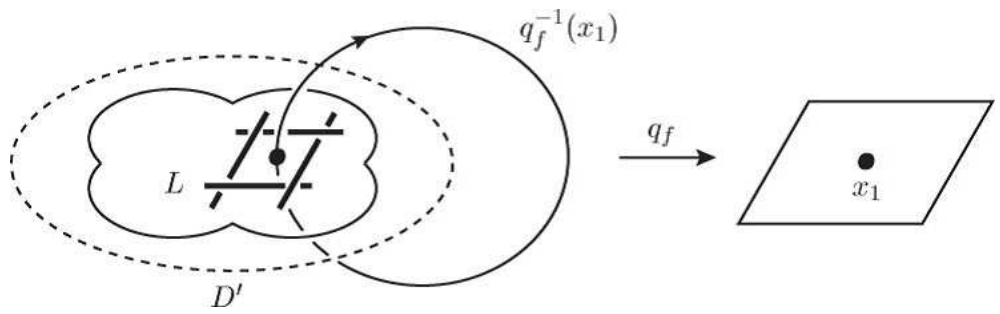

Figure 19. The configuration of a regular fiber.

(2) Let $x_{2}$ be a point in $L_{i} \times(0,1)$. Then the preimage $q_{f}^{-1}\left(x_{2}\right)$, which is also a regular fiber, is a meridian of $L_{i}$. See Figure 20.

(3) Let $x_{3}$ be a point in $L_{i} \times\{1\}$. Then the preimage $q_{f}^{-1}\left(x_{3}\right)$, which is a singular fiber of type $\mathrm{I}^{0}$, is a point in $L_{i}$. See Figure 20. 


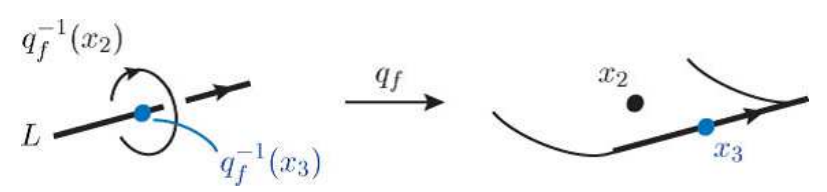

Figure 20. The configuration of singular fiber of type $\mathrm{I}^{0}$.

(4) Let $x_{4}$ be a point in $S\left(P_{D_{L}}\right) \backslash V\left(P_{D_{L}}\right)$. Let $\alpha$ be a transverse arc for $f$ at $\bar{f}\left(x_{4}\right)$. Then the preimage $Y=\bar{f}^{-1}(\alpha)$ is a Y-shaped graph. One endpoint of $Y$, say $y_{1}$, is mapped to one endpoint of $\alpha$ by $\bar{f}$ while the remaining two endpoints, say $y_{2}$ and $y_{3}$, are mapped to the other endpoint of $\alpha$. The preimage $q_{f}^{-1}(Y)$ is a pair of pants spanned by the three circles $q_{f}^{-1}\left(y_{1}\right), q_{f}^{-1}\left(y_{2}\right)$ and $q_{f}^{-1}\left(y_{3}\right)$. Note that the configuration of these circles has already explained in Cases (1) and (2). Then the preimage $q_{f}^{-1}\left(x_{4}\right)$, which is a singular fiber of type $\mathrm{I}^{1}$, lies in $q_{f}^{-1}(Y)$ as a spine as illustrated in Figure 21.

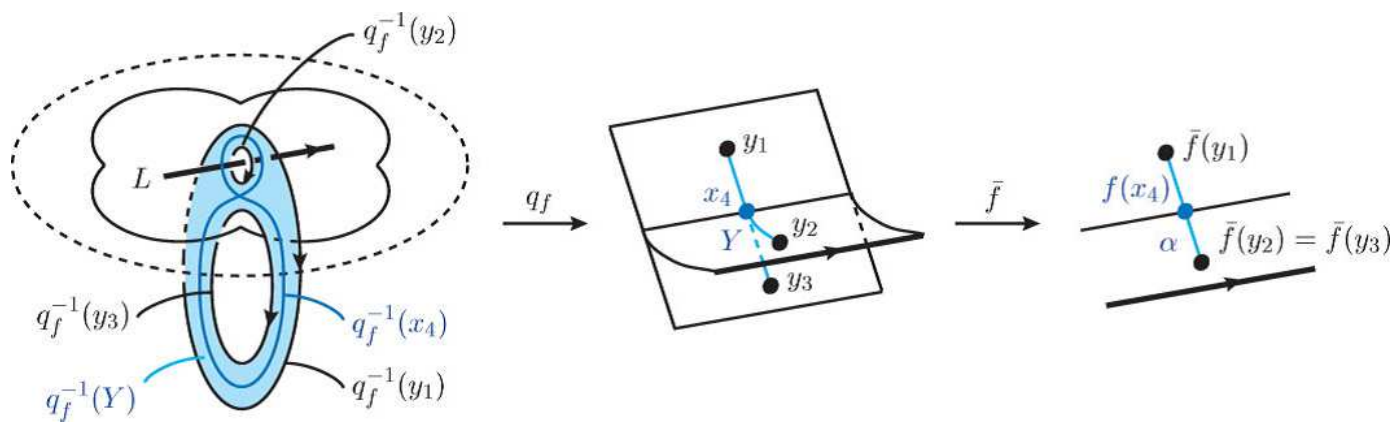

Figure 21. The configuration of singular fiber of type $\mathrm{I}^{1}$.

(5) Finally let $x_{5}$ be a true vertex of $P_{D_{L}}$. Let $\alpha$ be a transverse arc for $f$ at $\bar{f}\left(x_{5}\right)$ as shown in Figure 22. Then the preimage $X=\bar{f}^{-1}(\alpha)$ is the $X$-shaped graph, i.e., the cone on four points. One endpoint of $X$, say $y_{1}$, is mapped to one endpoint of $\alpha$ by $\bar{f}$ while the remaining three endpoints, say $y_{2}, y_{3}$ and $y_{4}$, are mapped to the other endpoint of $\alpha$. The preimage $q_{f}^{-1}(X)$ is a disk with three holes spanned by the four circles $q_{f}^{-1}\left(y_{1}\right), q_{f}^{-1}\left(y_{2}\right), q_{f}^{-1}\left(y_{3}\right)$ and $q_{f}^{-1}\left(y_{4}\right)$. Note, again, that the configuration of these circles has already explained in Cases (1) and (2). Then the preimage $q_{f}^{-1}\left(x_{5}\right)$, which is a singular fiber of type $\mathrm{II}^{2}$, lies in $q_{f}^{-1}(X)$ as a spine as illustrated in Figure 22.

Let $L$ be a link in $S^{3}$. Recall that the crossing number of $L$, denoted by $\operatorname{cr}(L)$, is the minimal number of crossings in any of its diagrams on $\mathbb{R}^{2}$.

Lemma 3.1. Let $L$ be an oriented non-split link in $S^{3}$. There exists a diagram $D_{L}$ for $L$ on an oriented disk with $\operatorname{cr}(L)$ crossing points such that $D_{L}$ becomes admissible after reversing the orientations of all link components if necessary. 


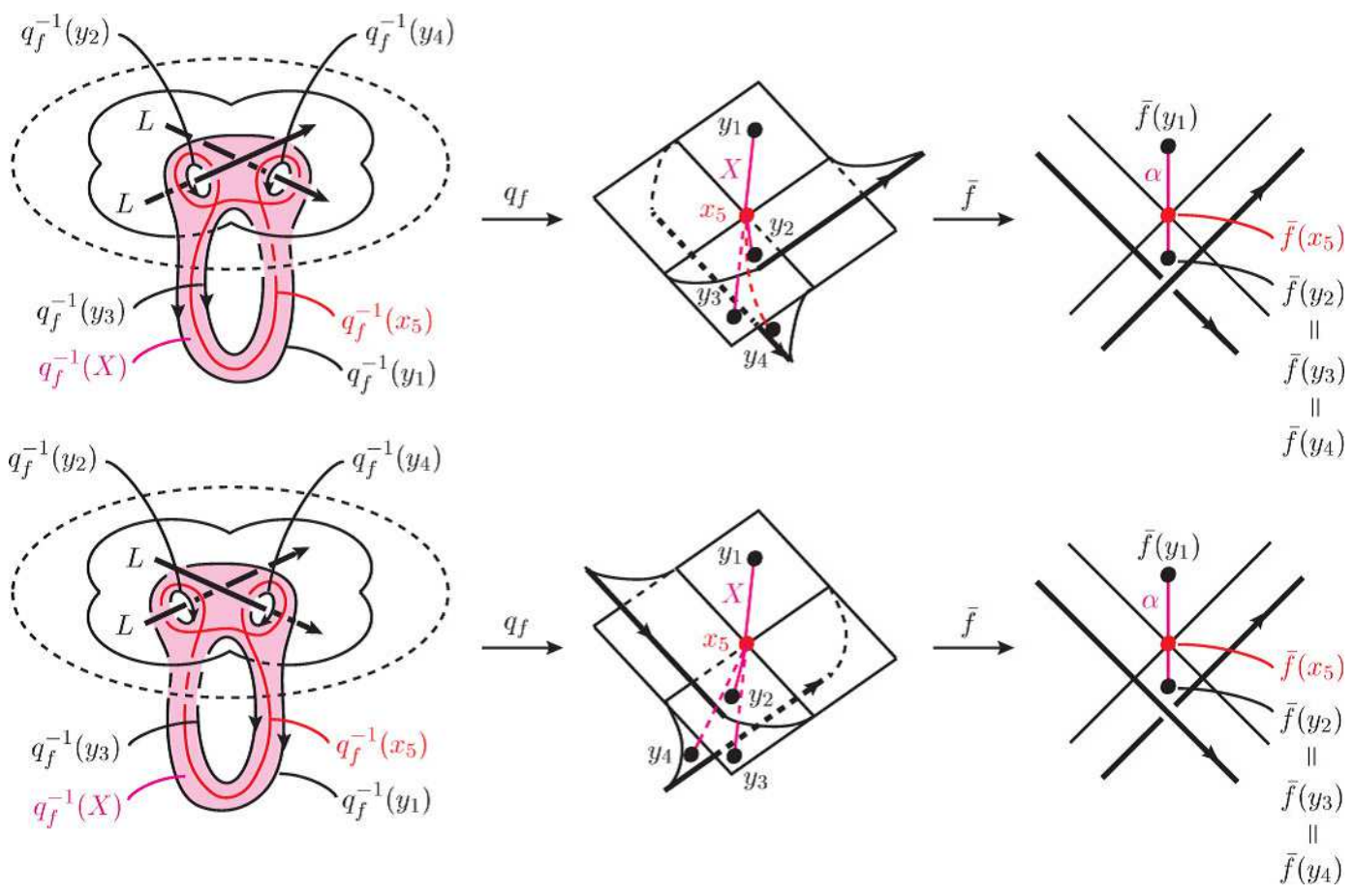

FiguRE 22. The configuration of singular fiber of type $\mathrm{II}^{2}$.

Proof. We start with a diagram $D_{L}$ for $L$ on the oriented 2-sphere $S^{2}$ with minimal crossing number. Using Seifert's algorithm for $D_{L}$ we get a disjoint collection $\left\{c_{1}, c_{2}, \ldots, c_{m}\right\}$ of circles on $S^{2}$. We refer to, for instance, Rolfsen [35] for Seifert's algorithm. Let $d$ be an open disk component of $S^{2} \backslash \bigcup_{i=1}^{m} c_{i}$. Let $D$ be a disk obtained by removing a small open neighborhood of an interior point of $d$. We equip $D$ with the orientation induced by that of $S^{2}$. Then the diagram $D_{L}$ on $D$ with the orientation of $L$ or its inverse, is the required diagram for $L$.

The next theorem immediately follows from the above argument and Lemma 3.1 .

Theorem 3.2. For a link $L$ in $S^{3}$, there exists a proper stable map $f:\left(S^{3}, L\right) \rightarrow \mathbb{R}^{2}$ without cusp points such that

(1) the Stein factorization $W_{f}$ is contractible; and

(2) $\left|\mathrm{II}^{2}(f)\right| \leqslant \operatorname{cr}(L)-2$ and $\mathrm{II}^{3}(f)=\emptyset$.

Further, the configuration of both the regular and singular fibers of $f$ can be described using a diagram of $L$.

Example 3 . The left-hand side in Figure 23 shows an admissible diagram $D_{K}$ of the figureeight knot $K$. The right-hand side in the figure illustrates the contractible branched polyhedron $P_{D_{K}}$ constructed from $D_{K}$. Then we have a proper stable map $f:\left(S^{3}, K\right) \rightarrow$ $\mathbb{R}^{2}$ with Stein factorization $\left(S^{3}, K\right) \stackrel{q_{f}}{\longrightarrow} P_{D_{K}} \stackrel{\bar{f}}{\longrightarrow} \mathbb{R}^{2}$ such that $\left|\mathrm{II}^{2}(f)\right|=2, \mathrm{II}^{3}(f)=\emptyset$ 
and $C(f)=\emptyset$. The configuration of the preimages of the points $x_{1}, x_{2}, \ldots, x_{6}$ in $P_{D_{K}}$ is shown in the figure.

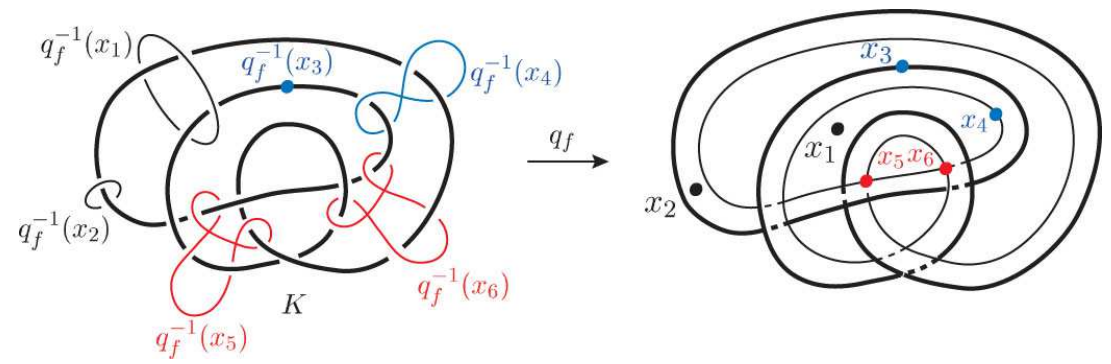

FiguRE 23. Regular and singular fibers of a stable map of the figureeight knot. $q_{f}^{-1}\left(x_{1}\right)$ and $q_{f}^{-1}\left(x_{2}\right)$ are regular fibers of $f . q_{f}^{-1}\left(x_{3}\right)$ is a singular fiber of type $\mathrm{I}^{0} \cdot q_{f}^{-1}\left(x_{4}\right)$ is a singular fiber of type $\mathrm{I}^{1} \cdot q_{f}^{-1}\left(x_{5}\right)$ and $q_{f}^{-1}\left(x_{6}\right)$ are singular fibers of type $\mathrm{II}^{2}$.

From Corollary 2.10 and Theorem 3.2, the following holds:

Corollary 3.3. Let $M$ be a closed orientable 3-manifold obtained from $S^{3}$ by surgery along a link $L \subset S^{3}$. Then there exists a stable map $f: M \rightarrow \mathbb{R}^{2}$ without cusp points such that $\left|\mathrm{II}^{2}(f)\right| \leqslant \operatorname{cr}(L)-2$ and $\mathrm{II}^{3}(f)=\emptyset$.

Remark 3.4. A result similar to Corollary 3.3 is obtained in Kalmár-Stipsicz [18, Theorem 1.2]. The numbers of singular fibers of types $\mathrm{II}^{2}$ and $\mathrm{II}^{3}$, and cusp points in our Corollary 3.3 are less than theirs (in particular, $C(f)=\emptyset$ in our result).

\section{Knots With BRANCHED SHADOW COMPLEXITY 1}

In this section, we use the arguments in the previous section to characterize hyperbolic links in $S^{3}$ whose exteriors have stable map complexity 1 . .

Lemma 4.1. Let $P$ be a shadow of a compact orientable 3-manifold $M$. Suppose that $P$ is embedded in a compact orientable smooth 4-manifold $W$ in a locally flat way so that $W \backslash P \cong \partial W \times(0,1]$. Let $\pi: M \rightarrow P$ be a projection given by a collapsing $W \searrow P$. Then the homomorphism $\pi_{1}(M) \rightarrow \pi_{1}(P)$ induced by $\pi$ is an epimorphism.

Proof. The proof follows immediately from the fact that every closed curve $l$ in $P$ can be lifted to a closed curve $\tilde{l}$ in $M$.

In the following, let $U_{1}, U_{2}, U_{3}$ and $U_{4}$ be the diagrams of the trivial knot illustrated in Figure 24.

Lemma 4.2. Let $M$ be a closed orientable 3-manifold that does not contain non-separating tori, and let $P$ be a shadow of $M$. Then any simple closed curve in a region of $P$ separates $P$. 


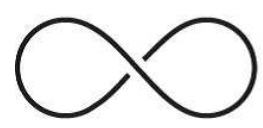

$U_{1}$

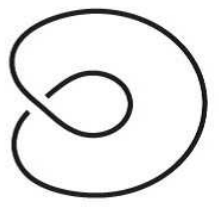

$U_{2}$

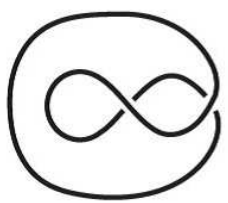

$U_{3}$

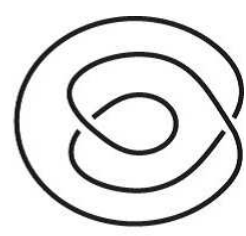

$U_{4}$

Figure 24. The diagrams $U_{1}, U_{2}, \ldots, U_{4}$ of the trivial knot.

Proof. Let $\pi: M \rightarrow P$ be a projection as in Lemma 4.1. If a simple closed curve $l$ in a region of $P$ does not separate $P$, then $\pi^{-1}(l)$ is a non-separating torus in $M$, which is a contradiction.

Theorem 4.3. Let $K$ be a hyperbolic knot in $S^{3}$. Then $\left(S^{3}, K\right)$ admits a branched shadow $P$ such that $c(P)=1$ and $S(P)$ is connected if and only if $K$ is one of the knots shown in Figure 25.

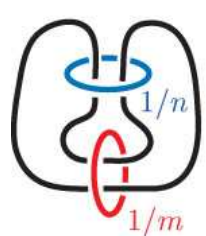

$K_{1}(m, n)$

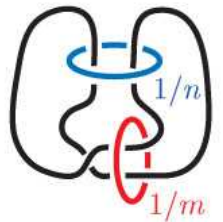

$K_{2}(m, n)$

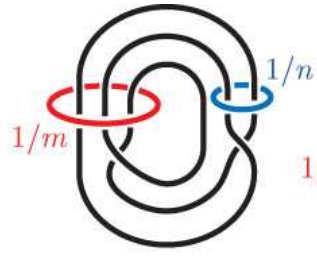

$K_{3}(m, n)$

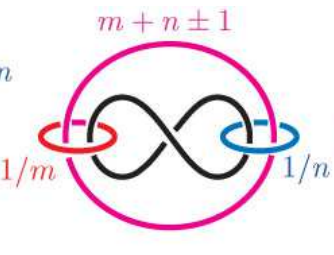

$K_{4}^{ \pm}(m, n)$

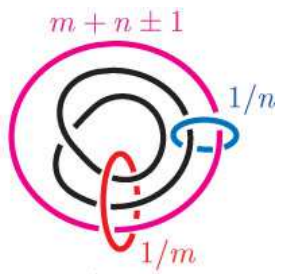

$K_{5}^{ \pm}(m, n)$

FiguRE 25. The knots admitting a branched shadow with one vertex and connected singular set.

Remark 4.4. Using Conway's normal form, the knots $K_{1}(m, n)$ and $K_{2}(m, n)$ can be written as $C(2 n, 2 m)$ and $C(2 n, 2 m+1)$, respectively. Also, $K_{4}^{ \pm}(m, n)$ is a knot obtained by performing Dehn surgery on one component of $C(2 n+1,2 m+1)$ with the coefficients $m+n \pm 1$. The knot $K_{3}(m, n)$ is the twisted torus knot $T(3,3 m+2 ; 2, n)$, see CallahanDean-Weeks [6] for the definition.

Proof. Suppose that $\left(S^{3}, K\right)$ admits a branched shadow $P$ such that $c(P)=1$ (so $|V(P)|=1$ and $|B V(P)|=0)$ and $S(P)$ is connected. Then $S(P)$ is one of the two graphs shown in Figure 26. Suppose that $S(P)$ is the one shown on the left-hand side
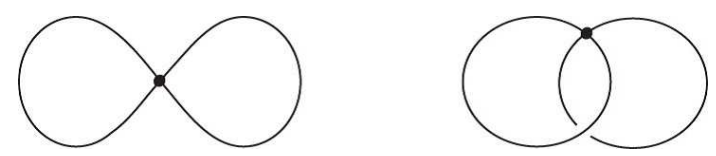

Figure 26. Two types of $S(P)$.

in the figure. Then its neighborhood $\operatorname{Nbd}(S(P) ; P)$ in $P$ is isomorphic to one of the 


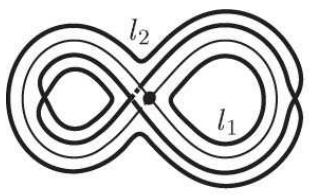

(i)

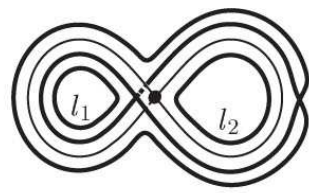

(ii)

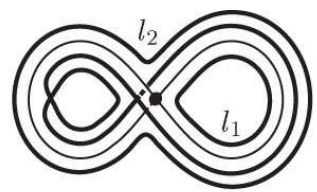

(iii)

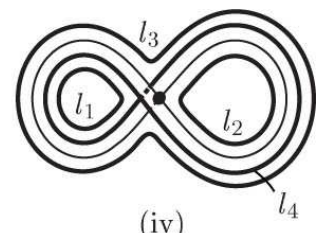

(iv)

Figure 27. Four possible shapes of $\operatorname{Nbd}(S(P) ; P)$.

four models illustrated in Figure 27. By Lemmas 4.1 and 4.2, each loop of the boundary of $\operatorname{Nbd}(S(P) ; P)$ separates $P$, and $P$ is a simply connected polyhedron obtained from $\operatorname{Nbd}(S(P) ; P)$ by capping off some of the boundary components by disks. Case (i) is impossible because we can not obtain a simply connected polyhedron no matter how we cap off boundary components of $\operatorname{Nbd}(S(P) ; P)$ by disks.

In Case (ii), $P$ is simply connected if and only if $P$ is obtained from $\operatorname{Nbd}(S(P) ; P)$ by capping off the boundary components $l_{1}$ and $l_{2}$ by disks. We denote the two disk regions corresponding to $l_{1}$ and $l_{2}$ by $D_{1}$ and $D_{2}$, respectively. Then as a branched polyhedron $P$ is isomorphic to $P_{U_{3}}$, see the left-hand side in Figure 28, Note that in this case, the
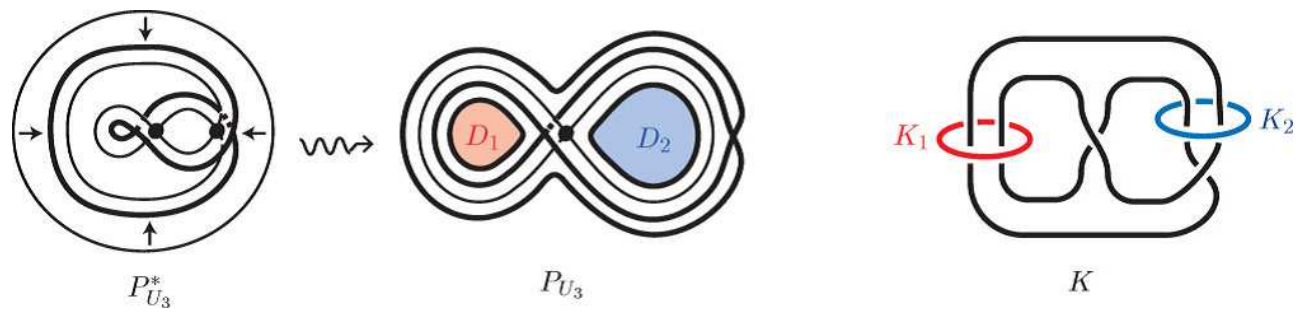

Figure 28. The link in Case (ii) in Figure 27.

regions $D_{1}$ and $D_{2}$ are equipped with the gleams $1 / 2$ and 1 , respectively. Moreover, we note that for each $i \in\{1,2\}$, the preimage of an interior point of $D_{i}$ under the projection $\pi: S^{3} \rightarrow P$ is a trivial loop $K_{i}$ illustrated on the right-hand side in Figure 28. In general, the gleams of the regions $D_{1}$ and $D_{2}$ are $1 / 2+m$ and $1+n$, respectively, where $m, n$ are integers. Then the shadowed branched polyhedron $P$ is a branched shadow of a knot $K$ shown on the right-hand side in Figure 28, where we perform the Dehn surgeries on $K_{1}$ and $K_{2}$ with the coefficients $1 / m$ and $1 / n$, respectively. Hence $K$ is the knot $K_{2}(m, n)$ shown in Figure 25.

In Case (iii) $P$ is simply connected if and only if $P$ is obtained from $\operatorname{Nbd}(S(P) ; P)$ by capping off the boundary components $l_{1}$ and $l_{2}$ by disks. Then as a branched polyhedron $P$ is isomorphic to $P_{U_{4}}$. In the same manner as in Case (ii), we can see that $K$ is the knot $K_{3}(m, n)$ shown in Figure 25.

In Case (iv), $P$ is simply connected if and only if $P$ is obtained from $\operatorname{Nbd}(S(P) ; P)$ in one of the following ways: 
(1) Cap off the boundary components $l_{i}$ and $l_{j}$ by disks, where $\{i, j\}=\{1,2\},\{1,3\}$, $\{1,4\},\{2,3\}$, or $\{2,4\}$.

(2) Cap off the boundary components $l_{i}, l_{j}$ and $l_{k}$ by disks, where $\{i, j, k\}=\{1,2,3\}$, $\{1,2,4\},\{1,3,4\}$ or $\{2,3,4\}$.

In Case (1), $P$ is isomorphic to $P_{U_{1}}^{*}$ or $P_{U_{2}}^{*}$ and hence it is easily seen that $K$ is one of the knots shown in Figure 29. It follows that $K$ is the trivial knot or the $(2, m+1)$-torus

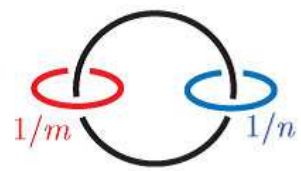

$K$

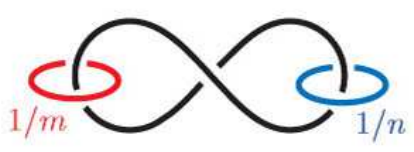

$K$

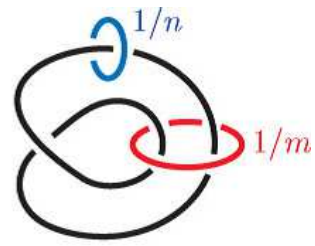

K

Figure 29. The links in Case(iv)-(1).

knot, where $m$ is an integer. This contradicts the assumption that $K$ is hyperbolic. Next we consider Case (2). If $\{i, j, k\}=\{1,2,3\}$, then $P$ is obtained by capping off the boundary component of $P_{U_{1}}^{*}$ that does not correspond to the trivial knot $U_{1}$ by a disk. We denote the two disk regions of $P_{U_{1}}^{*}$ by $D_{1}$ and $D_{2}$. We note that the both gleams on $D_{1}$ and $D_{2}$ defined by the construction of $P_{U_{1}}^{*}$ are $+1 / 2$. The two boundary circles $K$ and $K^{\prime}$ of $P_{U_{1}}^{*}$ together with the preimages $K_{1}$ and $K_{2}$ of interior points of $D_{1}$ and $D_{2}$, respectively, under the projection $\pi: S^{3} \rightarrow P$ form the minimally twisted 4-chain link. The changing of the gleams on $D_{1}$ and $D_{2}$ to $1 / 2+m$ and $1 / 2+n$, respectively, where $m, n$ are integers, corresponds to Dehn surgeries on $K_{1}$ and $K_{2}$ with the coefficients $1 / m$ and $1 / n$, respectively. Moreover, the capping off of $K^{\prime} \subset \partial P_{U_{1}}^{*}$ by a disk corresponds to a Dehn surgery on $K^{\prime}$ with the coefficients $p$, where $p$ is an integer, see Figure 30 . Hence

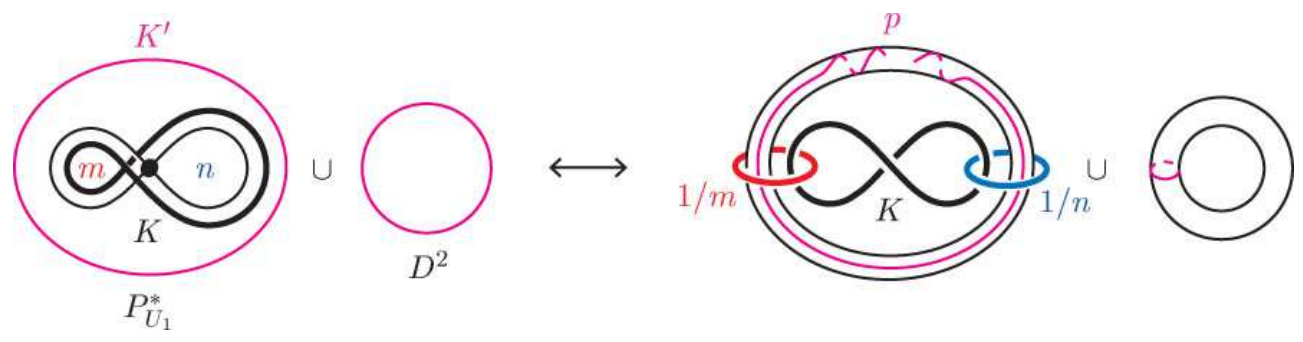

Figure 30. The link in the case $\{i, j, k\}=\{1,2,3\}$ or $\{1,2,4\}$.

$P$ is a branched shadow of a knot in $L(p-m-n, 1)$, thus $p=m+n \pm 1$ by assumption. In this case, $K$ is the knot $K_{4}^{ \pm}(m, n)$ shown in Figure 25. The same arguments apply to the case $\{i, j, k\}=\{1,2,4\}$. If $\{i, j, k\}=\{1,3,4\}$ or $\{2,3,4\}$, then $P$ is obtained by capping off the boundary component of $P_{U_{2}}^{*}$ that does not correspond to the trivial knot $U_{2}$ by a disk. By a similar argument as above, $K$ is a knot described in Figure 31, where 
$m, n, p$ are integers. Again, $P$ is a branched shadow of a knot in $L(p-m-n, 1)$, thus

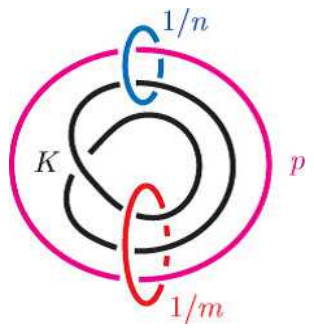

Figure 31. The link in the case $\{i, j, k\}=\{1,3,4\}$ or $\{2,3,4\}$.

$p=m+n \pm 1$ by assumption. In this case, $K$ is the knot $K_{5}^{ \pm}(m, n)$ shown in Figure 25.

Next, suppose that $S(P)$ is the one shown on the right-hand side in Figure 26. Then its neighborhood $\operatorname{Nbd}(S(P) ; P)$ in $P$ is homeomorphic to one of the four models illustrated in Figure 32. None of Cases (i)-(iii) is possible because we can not obtain a simply

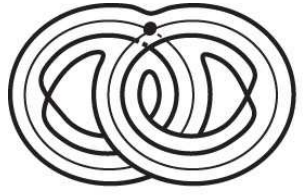

(i)

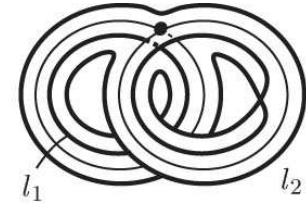

(ii)

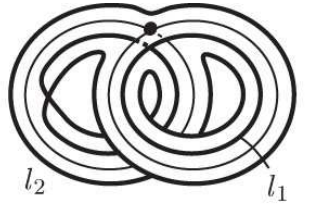

(iii)

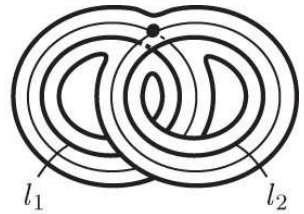

(iv)

Figure 32. The other four shapes of $\operatorname{Nbd}(S(P) ; P)$.

connected polyhedron no matter how we cap off boundary components of $\operatorname{Nbd}(S(P) ; P)$ by disks.

In Case (iv), $P$ is simply connected if and only if $P$ is obtained from $\operatorname{Nbd}(S(P) ; P)$ by capping off the boundary components $l_{1}$ and $l_{2}$ by disks. We denote the two disk regions corresponding to $l_{1}$ and $l_{2}$ by $D_{1}$ and $D_{2}$, respectively. We may embed $P$ into $S^{3}$ in such a way that $P$ is a spine of $S^{3}$, that is, $S^{3}$ collapses onto $P$ after removing a small open 3ball. Then the 3 -thickening of $P$ can be realized as a regular neighborhood $\operatorname{Nbd}\left(P ; S^{3}\right)$, which is homeomorphic to a closed 3-ball. We first consider the case where the both gleams of $D_{1}$ and $D_{2}$ are 0 . As observed in Costantino-Thurston [12, Lemma 3.24], the 4 -manifold reconstructed from $P$ is $\operatorname{Nbd}\left(P ; S^{3}\right) \times[-1,1] \cong B^{4}$ and hence $P$ is a branched shadow of $S^{3}$. Moreover, the boundary of $B^{4}$, which is $S^{3}$, can be identified with the union of $\operatorname{Nbd}\left(P ; S^{3}\right)$ and its mirror image glued along their boundaries by the canonical identity. The collapsing $B^{4} \rightarrow P$ is obtained by combining two collapsings $\operatorname{Nbd}\left(P ; S^{3}\right) \searrow P$ and $[0,1] \searrow\{0\}$. Let $k_{1}$ and $k_{2}$ be the preimages of interior points of $D_{1}$ and $D_{2}$, respectively, under the collapsing $\operatorname{Nbd}\left(P ; S^{3}\right) \searrow P$. Then on the boundary of $B^{4}$, the two copies of each $k_{i}$ give rise to a simple closed curve $K_{i}$ as described in Figure 33. In particular, we see that $K \sqcup K_{1} \sqcup K_{2}$ is the Borromean link. In the case where the gleams of $D_{1}$ and $D_{2}$ are $m$ and $n$, respectively, where $m, n$ are integers, $K$ is 


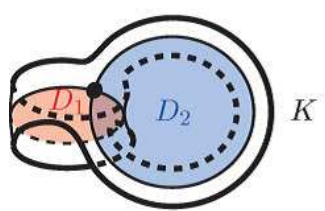

$\operatorname{Nbd}\left(P ; S^{3}\right)$

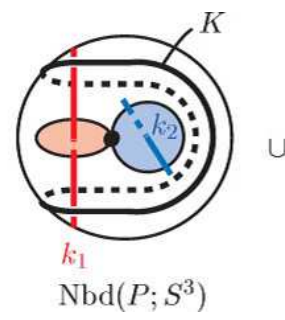

$\operatorname{Nbd}\left(P ; S^{3}\right)$

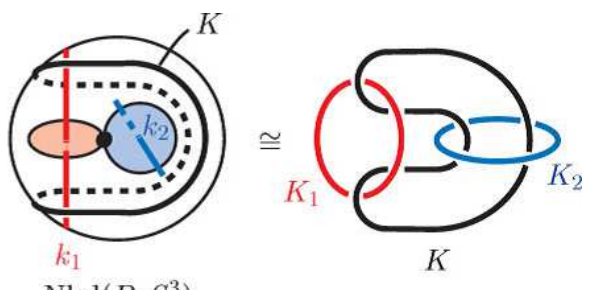

$\operatorname{Nbd}\left(P ; S^{3}\right)$

Figure 33. The link in Case (iv) in Figure 32

the knot in $S^{3}$ obtained by the Dehn surgeries on $K_{1}$ and $K_{2}$ with the coefficients $1 / \mathrm{m}$ and $1 / n$, respectively. Hence $K$ is the knot $K_{1}(m, n)$ shown in Figure 25.

Conversely, suppose that $K \subset S^{3}$ is a hyperbolic knot shown in Figure 25, By the above arguments, $\left(S^{3}, K\right)$ admits a branched shadow $P$ such that $c(P)=1$ and $S(P)$ is connected. This completes the proof.

We call the almost-special polyhedron of the shape depicted in Figure 34 a tower. We

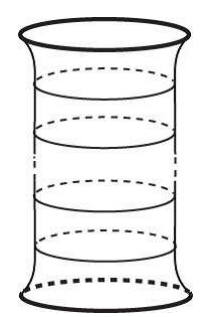

Figure 34. A tower.

require that a tower contains at least one disk region.

Lemma 4.5. Let $L$ be a hyperbolic link in $S^{3}$ with $\operatorname{bsc}\left(S^{3}, L\right)=1$, and let $P$ be a branched shadow of $\left(S^{3}, L\right)$ with $c(P)=1$. Let $P^{\prime}$ be a regular neighborhood of the component of $S(P)$ containing the vertex. Then there exists a branched shadow $P^{\prime \prime}$ of $\left(S^{3}, L\right)$ which is obtained from $P^{\prime}$ by attaching towers to some of its boundary components.

Proof. Let $l$ be a component of $\partial P^{\prime}$. By Lemma 4.2, $l$ separates $P$ into two components $P_{1}$ and $P_{2}$. Without loss of generality we can assume that $P_{1}$ is the component containing the vertex. It suffices to show that we can replace $P_{2}$ with a tower $Q$ so that the resulting shadowed polyhedron $P_{1} \cup_{l} Q$ is again a branched shadow of $\left(S^{3}, L\right)$. Let $\pi: S^{3} \rightarrow P$ be a projection as in Lemma 4.1. We set $M_{i}=\pi^{-1}\left(P_{i}\right)(i=1,2)$ and $T=\pi^{-1}(l)$. Then at least one of $M_{1}$ and $M_{2}$ is a solid torus.

Suppose that $T$ is parallel to $\partial \operatorname{Nbd}(K)$ in $E(L)$, where $K$ is a component of $L$. If $K$ is in $M_{1}$ then $M_{1} \cap L=K$, and hence $P_{2}$ is a branched shadow of $E(L)$. Since $P_{2}$ has no vertices, it follows from Lemma 2.9 and Proposition 2.11 that $E(L)$ is a graph manifold, which contradicts the hyperbolicity of $L$. If $K$ is in $M_{2}$ then $M_{2} \cap L=K$, hence by replacing $P_{2}$ with a tower, we have a branched shadow of $\left(S^{3}, L\right)$. 
Now we assume that $T$ is not parallel to any component of $\partial \operatorname{Nbd}(L)$. Suppose that $M_{i}$ is a solid torus, where $i$ is either 1 or 2 .

First we consider the case where the solid torus $M_{i}$ is knotted in $S^{3}$, that is, $M_{3-i}$ is not a solid torus. If $M_{i} \cap L$ is empty, we can attach a tower $Q$ to $P_{3-i}$ along $l$ so that the resulting polyhedron $P_{3-i} \cup_{l} Q$ is again a branched shadow of $\left(S^{3}, L\right)$ after giving suitable gleams to its regions. If $i=1$, then $\operatorname{bsc}\left(S^{3}, L\right)=0$, which contradicts the hyperbolicity of $L$. Hence we have $i=2$, which is our assertion. Suppose that $M_{i} \cap L$ is not empty. If $M_{i} \cap L$ is not contained in a 3-ball in $M_{i}$, then $T$ is an essential torus in $E(L)$. By Thurston's Hyperbolization Theorem [40, 28, 29, 30, 19] this implies $L$ is not hyperbolic, which is a contradiction. If $M_{i} \cap L$ is contained in a 3-ball in $M_{i}$, then either $L$ is a split link or $M_{3-i} \cap L=\emptyset$. The former case contradicts the hyperbolicity of $L$. In the latter case, we can attach a tower $Q$ to $P_{i}$ along $l$ so that the resulting polyhedron $P_{i} \cup_{l} Q$ is again a branched shadow of $\left(S^{3}, L\right)$ after giving suitable gleams to its regions. Note that the operation replacing $P_{3-i}$ by $Q$ corresponds to a Fox re-embedding of $M_{i}$, and under this re-embedding the knot type of $L$ does not change since $L$ is contained in a 3 -ball in $M_{i}$. If $i=2$, then $\operatorname{bsc}(L)=0$, which contradicts the hyperbolicity of $L$. Hence we have $i=1$, which is our assertion.

Next we consider the case where $M_{i}$ is unknotted, that is, $M_{3-i}$ is also a solid torus. Since $L$ is hyperbolic and $T$ is not parallel to any component of $\partial \operatorname{Nbd}(L)$, we have either $L \subset M_{1}$ or $L \subset M_{2}$, otherwise $T$ is an essential torus in $E(L)$ or $L$ is a split link. If $L \subset M_{2}$ then, attaching a tower to $P_{2}$, we obtain a branched shadow without vertices. Hence we have $L \subset M_{1}$. Then attaching a tower to $P_{1}$, we obtain a branched shadow of $\left(S^{3}, L\right)$, which is our assertion.

Theorem 4.6. Let $L$ be a hyperbolic link in $S^{3}$. Then $\operatorname{bsc}\left(S^{3}, L\right)=1$ if and only if the exterior of $L$ is diffeomorphic to a 3-manifold obtained by Dehn filling the exterior of one of the six links $L_{1}, L_{2}, \ldots, L_{6}$ in $S^{3}$ along some of (possibly none of) boundary tori of its exterior, where $L_{1}, L_{2}, \ldots, L_{6}$ are illustrated in Figure 2.

Proof. Let $P$ be a branched shadow of $\left(S^{3}, L\right)$ with one vertex. By Lemma 4.1, $P$ is simply connected. Let $P^{\prime}$ be a regular neighborhood of the component of $S(P)$ containing the vertex. Then $P^{\prime}$ is isomorphic to one of the eight models illustrated in Figures 27 and 32. By Lemma 4.5, we may assume that $P$ is obtained from $P^{\prime}$ by attaching towers to some of its boundary components. It follows from Seifert-van Kampen Theorem that $P$ can be simply connected if and only if $P^{\prime}$ is isomorphic to one of the eight models except Figure 32 (i). Thus it suffices to find a hyperbolic link $L$ in $S^{3}$ for each of the remaining seven models such that the shadow $P^{\prime}$ equipped with " $e$ " on each of the components of $\partial P^{\prime}$ is a branched shadow of the exterior $E(L)$. By Theorem 4.3, these links for Figure27 (ii), (iii), (iv) and Figure 32 (iv) are already obtained as follows: (Note that the links $L_{3}$, $L_{4}, L_{6}$ are those used to define the knots $K_{1}(m, n), K_{2}(m, n), K_{4}^{ \pm}(m, n)$, respectively, though each of them is depicted in a different way.)

(1) If $P^{\prime}$ is isomorphic to the model shown in Figure 27 (ii), then $P^{\prime}$ is a branched shadow of the exterior of $L_{4}$.

(2) If $P^{\prime}$ is isomorphic to the model shown in Figure 27 (iii), then $P^{\prime}$ is a branched shadow of the exterior of $L_{5}$. 
(3) If $P^{\prime}$ is isomorphic to the model shown in Figure 27 (iv), then $P^{\prime}$ is a branched shadow of the exterior of $L_{6}$.

(4) If $P^{\prime}$ is isomorphic to the model shown in Figure 32 (iv), then $P^{\prime}$ is a branched shadow of the exterior of $L_{3}$.

Suppose that $P^{\prime}$ is isomorphic to the model shown in Figure27(i). Then $P$ is obtained from $P^{\prime}$ by attaching towers to each of the boundary components $l_{1}, l_{2}$ of $P^{\prime}$, otherwise $P$ will not be simply-connected. Note that, in this case, $S(P)$ is not connected. Let $P_{0}$ be an almost-special polyhedron obtained by capping off $l_{1}$ and $l_{2}$ by disks, where $l_{1}$ and $l_{2}$ are the boundary components of $\operatorname{Nbd}(S(P) ; P)$ as shown in Figure 27 (i). Then as a polyhedron $P_{0}$ is a spine of $S^{3}$ that is called the abalone shown in Figure 35 See, for instance, Ikeda [16] and Matveev [27] for details of this spine. We denote the
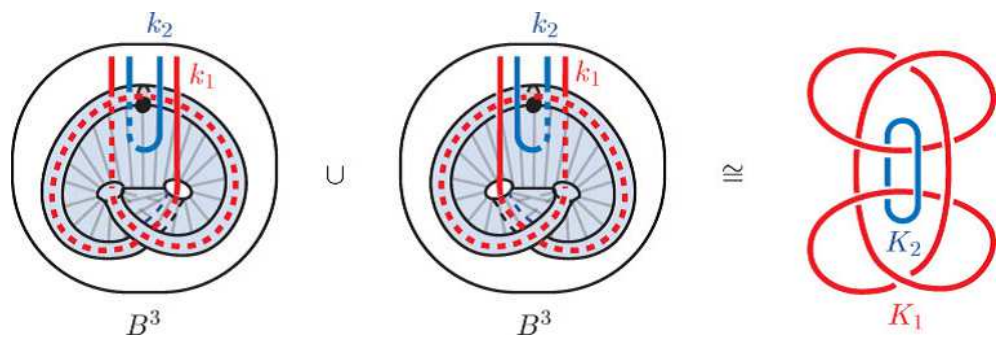

Figure 35. The link in Case (i) in Figure 27.

two disk regions corresponding to $l_{1}$ and $l_{2}$ by $D_{1}$ and $D_{2}$, respectively. If we assume that both gleams of $D_{1}$ and $D_{2}$ are 0 , then the 3-thickening of $P_{0}$ can be realized as a regular neighborhood $\operatorname{Nbd}\left(P_{0} ; S^{3}\right)$, which is homeomorphic to a closed 3-ball. Then as in the case of Figure 32 (iv) of Theorem 4.3, the 4-manifold reconstructed from $P_{0}$ is $\operatorname{Nbd}\left(P_{0} ; S^{3}\right) \times[-1,1] \cong B^{4}$ and hence $P_{0}$ is a branched shadow of $S^{3}$. Moreover, the boundary of $B^{4}$ can be identified with the union of $\operatorname{Nbd}\left(P_{0} ; S^{3}\right)$ and its mirror image glued along their boundaries by the canonical identity. The collapsing $B^{4} \searrow P$ is obtained by combining two collapsings $\operatorname{Nbd}\left(P_{0} ; S^{3}\right) \searrow P_{0}$ and $[0,1] \searrow\{0\}$. Let $k_{1}$ and $k_{2}$ be the preimages of interior points of $D_{1}$ and $D_{2}$, respectively, under the collapsing $\operatorname{Nbd}\left(P_{0} ; S^{3}\right) \searrow P_{0}$. Then on the boundary of $B^{4}$, the two copies of each $k_{i}$ give rise to a simple closed curve $K_{i}$ as described in Figure 35. Then the link $K_{1} \cup K_{2} \subset S^{3}$ is $L_{1}$. This implies that $P^{\prime}$ equipped with the color " $e$ " on each of the component of $\partial P^{\prime}$ is a branched shadow of the exterior $E\left(L_{1}\right)$.

We remark that Figure 32 (ii) and (iii) are isomorphic by the reflection. Suppose that $P^{\prime}$ is isomorphic to the model shown in Figure 32 (ii). Then $P$ is obtained from $P^{\prime}$ by attaching a tower to each of the boundary components $l_{1}, l_{2}$ of $P^{\prime}$, otherwise $P$ will not be simply-connected. Again in this case, $S(P)$ is not connected. Let $v$ be the vertex of $P^{\prime}$. Let $\operatorname{Nbd}(v ; P) \subset P^{\prime}$ be a regular neighborhood such that $P^{\prime} \backslash \operatorname{Nbd}(v ; P)$ consists of two components $P_{1}$ and $P_{2}$, where each of $P_{1}$ and $P_{2}$ is isomorphic to the direct product of a Y-shaped graph and an interval. We assume that $P_{1} \cap l_{1} \neq \emptyset\left(\right.$ so $\left.P_{2} \cap l_{1}=\emptyset\right)$. Then $\operatorname{Nbd}(v ; P) \cup P_{1}$ is a shadow of the 3-manifold $M_{1}$ shown in Figure 36 while $P_{2}$ is a shadow of the direct product $M_{2}$ of a pair of pants and an interval shown in the same 
figure. We may glue $M_{1}$ and $M_{2}$ along the pair of pants as indicated in the figure to
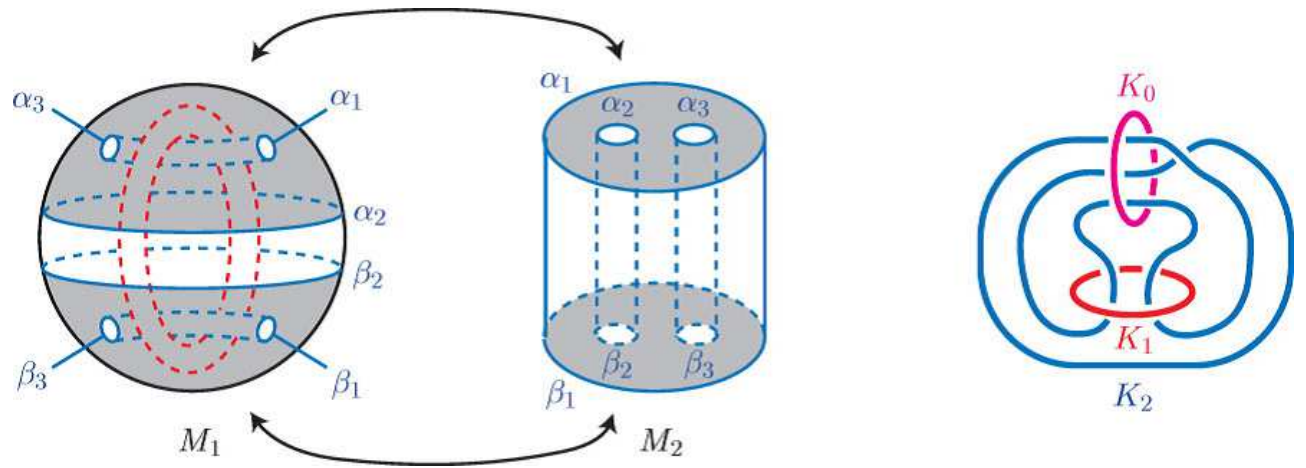

Figure 36. The link in Cases (ii) and (iii) in Figure 32.

get a 3-manifold $M$ presented by the branched shadow $P^{\prime}$. Let $K_{0} \cup K_{1} \cup K_{2}$ be the link shown on the right-hand side in Figure 36. It is easy to see that $M$ is the exterior of $K_{1} \cup K_{2}$ after performing Dehn surgery along $K_{0}$ with the coefficient 0 . We want to describe $M$ as the exterior of a link in $S^{3}$. To do so, we perform Dehn surgery along $K_{2}$ with the coefficient 1 . Let $K_{2}^{*}$ be the core of the solid torus corresponding to $K_{2}$ in the surgered manifold. Since $K_{0} \cup K_{2}$ is the Hopf link, the surgered manifold is $S^{3}$ and $K_{1} \cup K_{2}^{*}$ is a link whose exterior is $M$. By an elementary argument of Dehn surgery, we may check that the link $K_{1} \cup K_{2}^{*}$ is $L_{2}$.

Conversely, suppose that $L \subset S^{3}$ is a hyperbolic link whose exterior is diffeomorphic to a 3-manifold obtained by Dehn filling the exterior of one of the six links $L_{1}, L_{2}, \ldots, L_{6}$ in $S^{3}$ along some of (possibly none of) boundary tori of its exterior. By Proposition 2.11. $\operatorname{bsc}\left(S^{3}, L\right) \geqslant 1$. Moreover, by the above arguments, $\left(S^{3}, L\right)$ admits a branched shadow $P$ with $c(P)=1$. This completes the proof.

Remark 4.7. In Case (3) of the above proof, we can also use the 4-component link depicted in Figure 31 instead of $L_{6}$. Actually, this link and $L_{6}$ have the diffeomorphic complements.

From Theorems 2.2 and 4.6 , the following holds:

Corollary 4.8. Let $L$ be a hyperbolic link in $S^{3}$. Then there exists a stable map $f$ : $\left(S^{3}, L\right) \rightarrow \mathbb{R}^{2}$ without cusp points such that $\left|\mathrm{II}^{2}(f)\right|=1$ and $\mathrm{II}^{3}(f)=\emptyset$ if and only if the exterior of $L$ is diffeomorphic to a 3-manifold obtained by Dehn filling the exterior of one of the six links $L_{1}, L_{2}, \ldots, L_{6}$ in Theorem 4.6 along some of (possibly none of) boundary tori of its exterior.

Since the correspondence between S-maps and branched shadows are so explicit, we can completely determine the configuration of the singular fibers of the S-maps from the branched shadows. In the rest of this section, we show the configuration of singular fibers of type $\mathrm{II}^{2}$ for the links $L_{3}, L_{4}, L_{5}$ and $L_{6}$ in Theorem 4.6. We omit the other links $L_{1}$ and $L_{2}$ since the configuration seems to be more complicated. 
Corollary 4.9. For $i \in\{3,4,5,6\}$, the exterior of the link $L_{i}$ admits an $S$-map $f$ : $E\left(L_{i}\right) \rightarrow \mathbb{R}^{2}$ with $c(f)=1$ such that the configuration of the unique singular fiber of type $\mathrm{II}^{2}$ is shown in Figure 37.

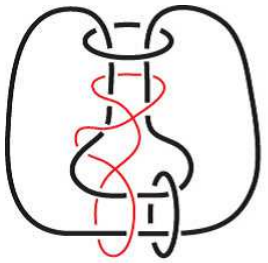

$L_{3}$

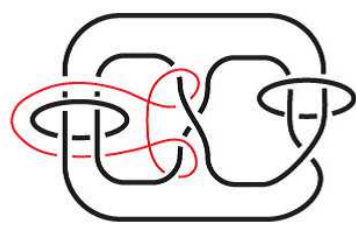

$L_{4}$

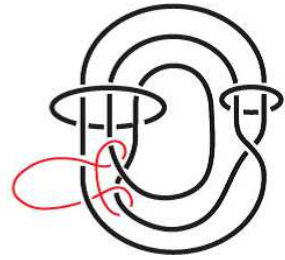

$L_{5}$

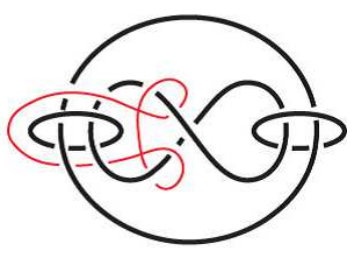

$L_{6}$

Figure 37. The configuration of singular fibers in Corollary 4.9.

Proof. Since the links $L_{4}, L_{5}, L_{6}$ admit branched shadows $P_{U_{3}}, P_{U_{4}}, P_{U_{1}}^{*}$, respectively, we get an S-map $f: E\left(L_{i}\right) \rightarrow \mathbb{R}^{2}(i \in\{4,5,6\})$ with $c(f)=1$ using these branched shadows and then we can find easily the configuration of its unique singular fiber of type $\mathrm{II}^{2}$ as shown in Figure 37, following the method introduced in Section 3 . Hence it remains to show the case of $L_{3}$. We recall that the exterior of $L_{3}$ admits a branched shadow $P^{\prime}$ depicted in Figure 32 (iv). We use the same notation as in the proof of Theorem 4.6, that is, $l_{1}$ and $l_{2}$ are the components of $\partial P^{\prime}$ shown in Figure 32 (iv) and $L_{3}$ consists of 3 components $K, K_{1}, K_{2}$ as shown in Figure 33 , Let $f: E\left(L_{3}\right) \rightarrow \mathbb{R}^{2}$ be an S-map with $c(f)=1$ constructed from the branched shadow depicted in Figure 32 (iv) using the argument in Theorem 2.2. Then $P^{\prime}$ can be naturally identified with $W_{f}$, where $E\left(L_{3}\right) \stackrel{q_{f}}{\longrightarrow} W_{f} \stackrel{\bar{f}}{\longrightarrow} \mathbb{R}^{2}$ is the Stein factorization of $f$. We denote the vertex of $P^{\prime}$ by $v$. Let $x_{1}, x_{2}, \ldots, x_{6}, y_{1}, y_{2}, \ldots, y_{6}$ be the points on $\partial \operatorname{Nbd}\left(v ; P^{\prime}\right) \cap \partial P^{\prime}$ shown on the left-hand side in Figure [38, and set $\partial \operatorname{Nbd}\left(v ; P^{\prime}\right) \cap S\left(P^{\prime}\right)=\left\{z_{1}, z_{2}, z_{3}, z_{4}\right\}$ as shown in the same figure. Let $Y_{i}(i \in\{1,2,3,4\})$ be the closure of the Y-shaped component of $\partial \operatorname{Nbd}\left(v ; P^{\prime}\right) \backslash \partial P^{\prime}$ containing $z_{i}$. Then by the argument in Theorem 4.3, $q_{f}^{-1}\left(x_{3}\right)$ and $q_{f}^{-1}\left(y_{3}\right)$ are longitudes of the trivial knot $K_{1}$, and $q_{f}^{-1}\left(x_{5}\right)$ and $q_{f}^{-1}\left(y_{5}\right)$ are longitudes of $K_{2}$, while all the other $x_{i}$ 's and $y_{i}$ 's are meridians of $K$. The preimage $Y_{i}(i \in\{1,2,3,4\})$ is a pair of pants spanned by the simple closed curves $q_{f}^{-1}\left(\partial Y_{i}\right)$ as shown on the right-hand side in Figure 38. We note that once we fix an orientation of $E\left(L_{3}\right)$ and $\mathbb{R}^{2}$, we may give an orientation of each of $q_{f}^{-1}\left(x_{i}\right)$ and $q_{f}^{-1}\left(y_{i}\right)(i \in\{1,2, \ldots, 6\})$ in a natural way. Then by the branching of the Stein factorization $P^{\prime}$, the preimage $q_{f}^{-1}\left(z_{i}\right)$, which is an immersed figure-8 shaped curve, lies in $q_{f}^{-1}\left(Y_{i}\right)$ as shown in the same figure. The union $\bigcup_{i=1}^{4} q_{f}^{-1}\left(Y_{i}\right)$ cuts $E\left(L_{3}\right)$ into a genus 3 handlebody $N_{0}$ and two genus 2 handlebodies $N_{1}, N_{2}$, where $N_{1}$ is bounded by $q_{f}^{-1}\left(Y_{1}\right)$ and $q_{f}^{-1}\left(Y_{2}\right)$, and $N_{2}$ is bounded by $q_{f}^{-1}\left(Y_{3}\right)$ and $q_{f}^{-1}\left(Y_{4}\right)$. We note here that each of $N_{1}$ and $N_{2}$ is the product (a pair of pants) $\times[0,1]$ corresponding to the preimage of one of the two components of the closure of $P^{\prime} \backslash \operatorname{Nbd}\left(v ; P^{\prime}\right)$ under $q_{f}$.

Now, the preimage $q_{f}^{-1}(v)$, which is our target, lies in $H_{0}$. As reviewed in Section 1.2 , $q_{f}^{-1}\left(\operatorname{Nbd}\left(v ; P^{\prime}\right)\right)$ has a standard product structure (a disk with 3 holes) $\times[0,1]$ for which 


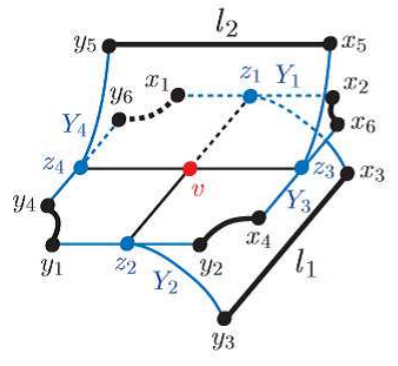

$\operatorname{Nbd}\left(v ; P^{\prime}\right)$

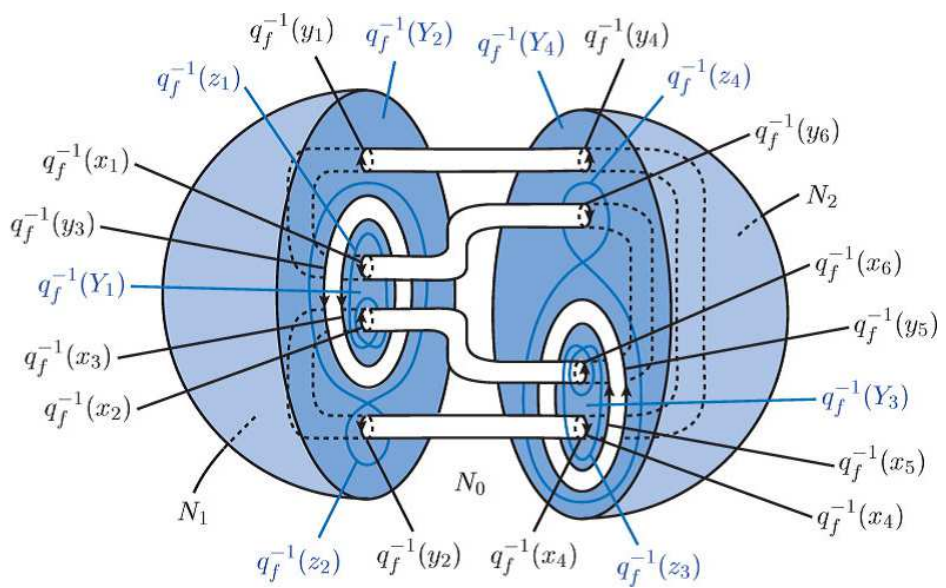

FiguRE 38. The configuration of singular fibers for $L_{3}$.

the preimages of $z_{1}, z_{2}, z_{3}, z_{4}$ and $v$ are illustrated on the left-hand side in Figure 39 (cf. Figure (8). The right-hand side in Figure 38 also gives a (a disk with 3 holes) $\times[0,1]$ of
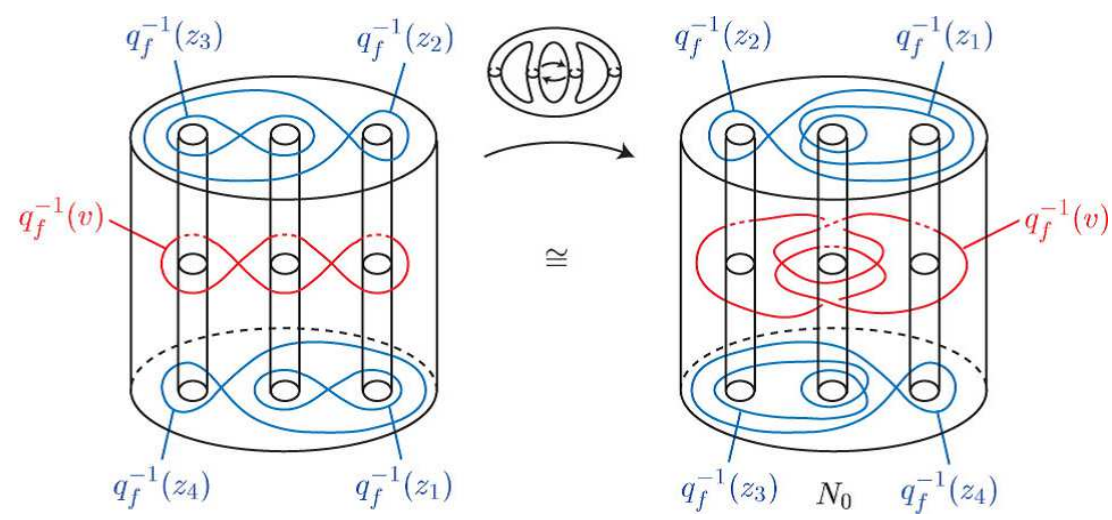

FIGURE 39. Twisting the handlebody.

the handlebody $H_{0}$ as shown on the right-hand side in Figure 39. However, this product structure is not consistent with the standard one. There exists an orientation-preserving diffeomorphism between the handlebodies, which is realized by switching the two handles as described in the figure. Using this diffeomorphism, we can find the configuration of $q_{f}^{-1}(v)$ inside $N_{0}$. This completes the proof.

Example 4. The knot $K_{2}(1,1)$ is the figure-eight knot. By Corollary 4.9 the exterior of $K_{2}(1,1)$ admits an S-map with $c(f)=1$ whose unique singular fiber of type $\mathrm{II}^{2}$ is shown in Figure 40, 


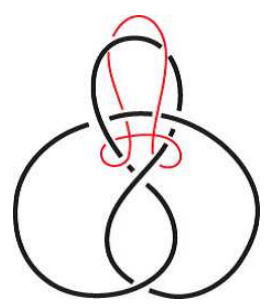

Figure 40. The configuration of the singular fiber of type $\mathrm{II}^{2}$ in the figure-eight knot complement.

\section{Stable maps AND hyperbolic VOlume}

Throughout the section, we consider a particular type of polyhedron, a special polyhedron. An almost-special polyhedron $P$ is said to be special if there is no loop without vertices in $S(P)$ and each region of $P$ is a disk. We note that in this case, $S(P)$ is connected and $P$ contains no boundary vertices. We call a shadow of a 3 -manifold that is a special polyhedron a special shadow of $M$. Remark that every closed orientable 3manifold admits a branched, special shadow by the moves described in Turaev [42] and Costantino [7]. See also Martelli [25] for interesting properties of special shadows.

Let $M$ be a closed orientable 3-manifold with special shadow $P$ and $\pi: M \rightarrow P$ be the projection induced by the collapsing $W \searrow P$, where $M=\partial W$. Set $M_{S(P)}=$ $\pi^{-1}(\operatorname{Nbd}(S(P) ; P))$. Costantino-Thurston [12] showed that $M_{S(P)}$ admits a complete, finite volume hyperbolic structure realized by gluing $2 c(P)$ copies of a regular ideal octahedron. Thus, in particular, we have $\operatorname{vol}\left(M_{S(P)}\right)=2 c(P) V_{\text {oct }}$. Since each region of a special polyhedron is a disk, $M$ is obtained from $M_{S(P)}$ by attaching solid tori, i.e., by Dehn fillings. In particular, by the 6-Theorem of Agol [2] and Lackenby [22] and the Geometrization Theorem of Perelman [31, 32, 33], if all slope lengths of the Dehn fillings are more than 6 then $M$ admits a complete finite volume hyperbolic structure. Since the hyperbolic structure of $M_{S(P)}$ is explicitly given by the ideal octahedra, the slope lengths of Dehn fillings can be calculated in terms of the combinatorial structure of the special polyhedron $P$ and the gleams on its regions.

Let $P$ be a shadowed, special polyhedron. For each region $R$ of $P$, set $\operatorname{sl}(R)=$ $\sqrt{(2 g)^{2}+k^{2}}$, where $g \in \frac{1}{2} \mathbb{Z}$ is the gleam on $R$ and $k$ is an integer counting how many times the boundary of the closure of $R$ passes through the vertices of $P$. We will show later, in Lemma 5.3, that $\operatorname{sl}(R)$ is nothing but the slope length of the Dehn filling for the corresponding boundary torus when we obtain $M$ from the hyperbolic manifold $M_{S(P)}$. We set $\operatorname{sl}(P)=\min _{R} \operatorname{sl}(R)$, where $R$ varies over all regions of $P$.

Proposition 5.1. Let $M$ be a closed orientable 3-manifold. Let $P$ be a branched, special shadow of $M$. If $\operatorname{sl}(P)>2 \pi$, then we have

$$
\begin{aligned}
2 \operatorname{smc}(M) V_{\text {oct }}\left(1-\left(\frac{2 \pi}{\operatorname{sl}(P)}\right)^{2}\right)^{3 / 2} & \leqslant 2 c(P) V_{\text {oct }}\left(1-\left(\frac{2 \pi}{\operatorname{sl}(P)}\right)^{2}\right)^{3 / 2} \\
& \leqslant \operatorname{vol}(M)<2 \operatorname{smc}(M) V_{\text {oct }}
\end{aligned}
$$


Proof. The first inequality is immediate from the definition. The second inequality follows from Theorem 2.2. Lemma 5.3 below, and Futer-Kalfagianni-Purcell [14, Theorem 1.1]. The last inequality follows from Costantino-Thurston [12, Theorem 3.37] and Theorem 2.2 .

From these inequalities we have the following result that concerns the coincidence of shadow complexities, branched shadow complexities and stable map complexities.

Theorem 5.2. Let $M$ be a closed orientable 3-manifold, and let $P$ be a branched, special shadow of $M$. If $\operatorname{sl}(P)>2 \pi \sqrt{2 c(P)}$, then we have $\operatorname{sc}(M)=\operatorname{bsc}(M)=\operatorname{smc}(M)=c(P)$.

Proof. The inequality $\operatorname{sl}(P)>2 \pi \sqrt{2 c(P)}$ implies that

$$
1-\frac{1}{c(P)}<1-2\left(\frac{2 \pi}{\mathrm{sl}(P)}\right)^{2}<\left(1-\left(\frac{2 \pi}{\mathrm{sl}(P)}\right)^{2}\right)^{3 / 2} .
$$

Thus we have the following inequalities:

$$
0<c(P)-c(P)\left(1-\left(\frac{2 \pi}{\operatorname{sl}(P)}\right)^{2}\right)^{3 / 2}<1 .
$$

Since $\operatorname{sc}(M), \operatorname{bsc}(M)$ and $\operatorname{smc}(M)$ are all less than or equal to $c(P)$, the above inequalities hold even if we replace $c(P)$ by $\operatorname{sc}(M), \operatorname{bsc}(M)$ and $\operatorname{smc}(M)$. Applying this to the inequalities in Proposition [5.1, we have

$$
0 \leqslant \operatorname{smc}(M)-\frac{\operatorname{vol}(M)}{2 V_{\text {oct }}}<1 .
$$

By the way, it is easy to check that the inequalities in Proposition 5.1 hold not only for the stable map complexity but also the shadow complexity, the branched shadow complexity and the number of vertices of the polyhedron $P$. Therefore the above inequality holds even if we replace $\operatorname{smc}(M)$ by $\operatorname{sc}(M), \operatorname{bsc}(M)$ and $c(P)$. Since $\operatorname{sc}(M), \operatorname{bsc}(M), \operatorname{smc}(M)$ and $c(P)$ are integers, they coincide.

Let $P$ be a special shadow of a closed orientable 3 -manifold $M$. Costantino-Thurston [12, Proposition 3.34] described the Euclidean structure on the cusps of $M_{S(P)}$ in terms of $\mathbb{Z}_{2}$-gleam and the numbers of the vertices through which the boundaries of the regions of $P$ runs. The proof of the following lemma is not essentially new. In fact, it is described implicitly in [12. The maximal horocusp had been observed in Costantino-FrigerioMartelli-Petronio 11 though gleams are excluded from the discussion. Therefore we believe that it deserves to be clarified in our setting with details.

Lemma 5.3. Let $P$ be a special shadow of a closed orientable 3 -manifold $M$. Let $R_{1}$, $R_{2}, \ldots, R_{n}$ be the regions of $P$. Set $l_{i}=R_{i} \cap \partial \operatorname{Nbd}(S(P) ; P)$, and let $T_{i}$ be the component of the boundary of $M_{S(P)}$ corresponding to $l_{i}$ for $i \in\{1,2, \ldots, n\}$. Then there exists a maximal horoball neighborhood $C=C_{1} \cup C_{2} \cup \cdots \cup C_{n}$ of the cusps of the interior of $M_{S(P)}$, where $C_{i}$ corresponds to $T_{i}$, such that $M$ is obtained from $M_{S(P)}$ by Dehn fillings along the slopes $s_{1} \subset T_{1}, s_{2} \subset T_{2}, \ldots, s_{n} \subset T_{n}$ whose lengths with respect to $C$ are $\operatorname{sl}\left(R_{1}\right)$, $\operatorname{sl}\left(R_{2}\right), \ldots, \operatorname{sl}\left(R_{n}\right)$, respectively. 
Proof. For each vertex $v_{i}(i \in\{1,2, \ldots, c(P)\})$, we set $P_{i}=\operatorname{Nbd}\left(v_{i} ; P\right)$. We regard $\operatorname{Nbd}(S(P) ; P)$ as the union $\bigcup_{i=1}^{c(P)} P_{i}$. For the 3-dimensional thickening $X_{i}$ of $P_{i}$, we fix a collapsing $\rho_{i}: X_{i} \searrow P_{i}$ so that:

- for a point $y$ in $P_{i} \backslash S\left(P_{i}\right), \rho_{i}^{-1}(y)=\{y\} \times[-1,1]$;

- for a point $y$ in $S\left(P_{i}\right) \backslash\left\{v_{i}\right\}, \rho_{i}^{-1}(y)$ is a Y-shaped graph; and

- $\rho_{i}^{-1}\left(v_{i}\right)$ is an X-shaped graph.

Then $\partial X_{i} \backslash \operatorname{Int}\left(\rho_{i}^{-1}\left(\partial P_{i}\right)\right)$ consists of four disks $d_{i, 1}, d_{i, 2}, d_{i, 3}$ and $d_{i, 4}$, and the closure of $\rho_{i}^{-1}\left(\partial P_{i}\right) \backslash \operatorname{Nbd}\left(\rho_{i}^{-1}(S(P))\right)$ consists of six squares $b_{i, 1}, b_{i, 2}, \ldots, b_{i, 6}$. We foliate each of these squares by intervals as shown in Figure 41, Set $W_{i}=X_{i} \times[-1,1]$. We note

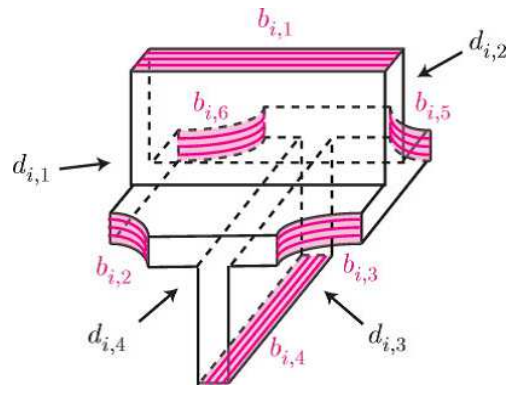

Figure $41 . P_{i}=\operatorname{Nbd}\left(v_{i} ; P\right)$.

that the 4-thickening $W_{0}$ of $\operatorname{Nbd}(S(P) ; P)$, which is the subbundle of the determinant line bundle over $X_{i}$ whose fiber is $[-1,1]$ (after giving an Euclidean metric over this bundle), is obtained by gluing the pieces $W_{1}, W_{2}, \ldots, W_{c(P)}$. Then the boundary $\partial W$ of the 4-thickening $W$ of $P$ decomposes as:

$$
W_{i} \cap \partial W=X_{i} \times\{-1\} \bigcup_{d_{i, j} \times\{-1\}}\left(\left(\bigsqcup_{i=1}^{4} d_{i, j}\right) \times[-1,1]\right) \bigcup_{d_{i, j} \times\{1\}} X_{i} \times\{1\} .
$$

See Figure 42, In the figure, $d_{i, j} \times\{ \pm 1\}(j \in\{1,2,3,4\})$ is denoted by $d_{i, j}^{ \pm}$and $b_{i, j} \times\{ \pm 1\}$ $(j \in\{1,2, \ldots, 6\})$ is denoted by $b_{i, j}^{ \pm}$. Moreover each $b_{i, j}^{ \pm}$inherits a foliation from $b_{i, j}$.

Let $X=\bigcup_{i=1}^{c(P)} X_{i}$ be the 3-dimensional thickening of $\operatorname{Nbd}(S(P) ; P)$. Let $\rho \searrow$ $\operatorname{Nbd}(S(P) ; P)$ be the collapsing given by $\rho_{i}(i \in\{1,2, \ldots, c(P)\})$, and let $\pi: W_{0} \searrow$ $\operatorname{Nbd}(S(P) ; P)$ be the collapsing given by $\rho$ and the projection $[-1,1] \rightarrow\{0\}$. Set $D_{i}=R_{i} \backslash \operatorname{Int} \operatorname{Nbd}(S(P), P)(i \in\{1,2, \ldots, c(P)\})$. We note that $P$ is obtained from $\operatorname{Nbd}(S(P) ; P)$ by attaching each disk $D_{i}$ along $l_{i}$. Suppose the boundary of the closure of $R_{i}$ runs $k_{i}$ times through the vertices of $P$. Then the preimage $\rho^{-1}\left(l_{i}\right)$, which is either an annulus or a Möbius band, is the union $\bigcup_{i=1}^{k_{i}} b_{\sigma(i), \tau(i)}$ with $\sigma(i) \in\{1,2, \ldots, c(P)\}$ and $\tau(i) \in\{1,2, \ldots, 6\}$. If $\rho^{-1}\left(l_{i}\right)$ is an annulus, it lies in the solid torus $\pi^{-1}\left(l_{i}\right)$ as shown in Figure 43. Then the annulus $\bigcup_{i=1}^{k_{i}} b_{\sigma(i), \tau(i)}$ is parallel to $\bigcup_{i=1}^{k_{i}} b_{\sigma(i), \tau(i)}^{\epsilon_{i}}$ in the solid torus $\pi^{-1}\left(l_{i}\right)$. Hence by the definition of gleams, attaching the disk $D_{i}$ to $P^{\prime}$ along $l_{i}$ and putting the gleam 0 on the resulting region $R_{i}$ correspond to performing 

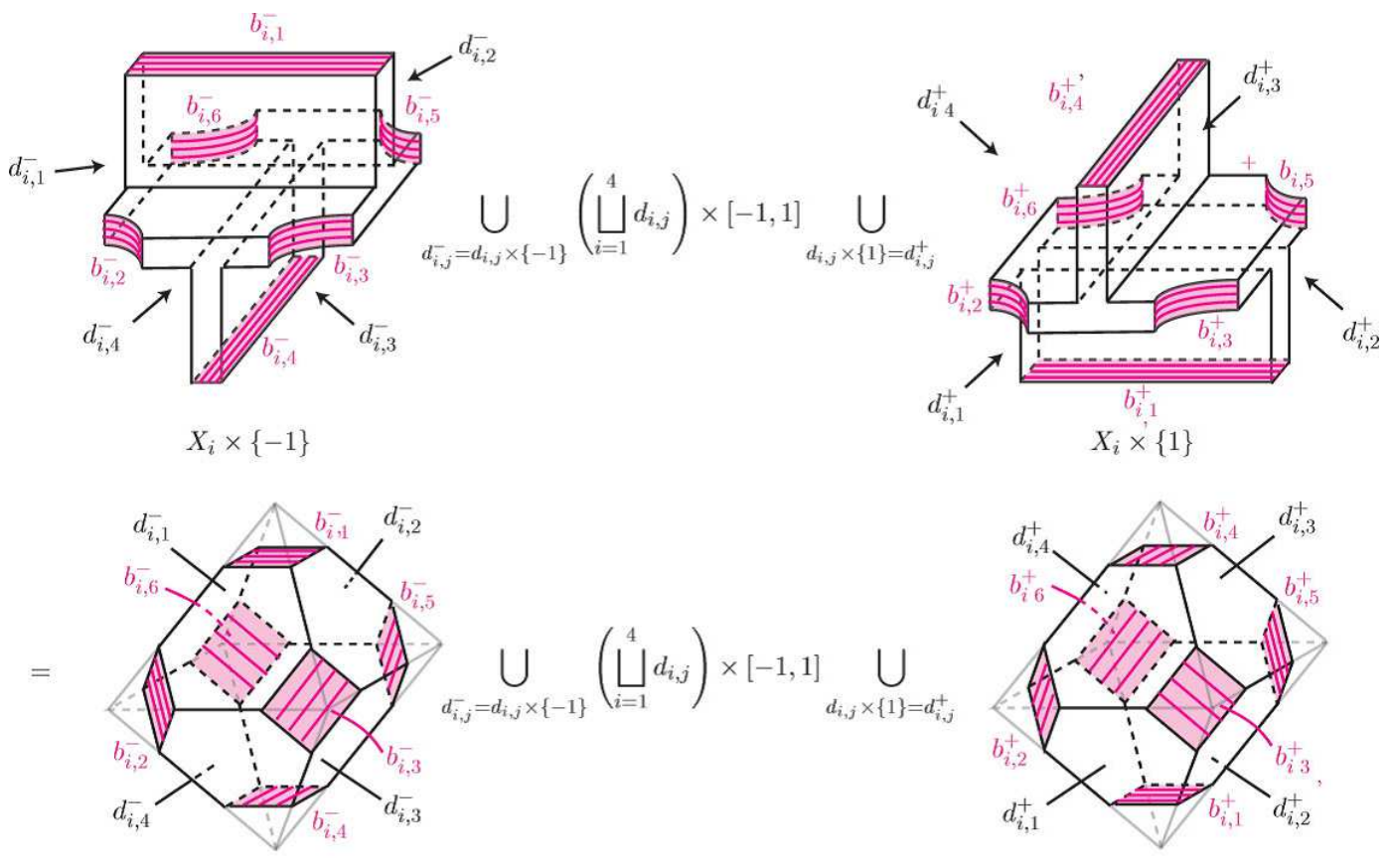

$$
X_{i} \times\{-1\}
$$

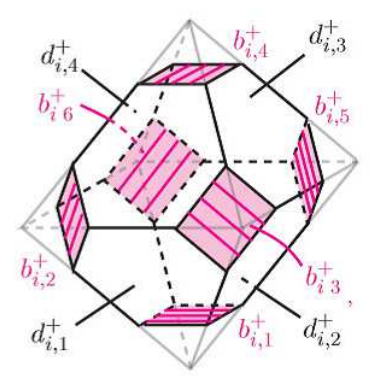

$X_{i} \times\{1\}$

Figure 42. $W_{i} \cap \partial W$. Topologically, the part $\left(\bigsqcup_{i=1}^{4} d_{i, j}\right) \times[-1,1]$ can be ignored and we may regard $W_{i} \cap \partial W$ as two truncated octahedra $X_{i} \times\{ \pm 1\}$ glued as $d_{i, j}^{-}=d_{i, j}^{+}(j \in\{1,2,3,4\})$.

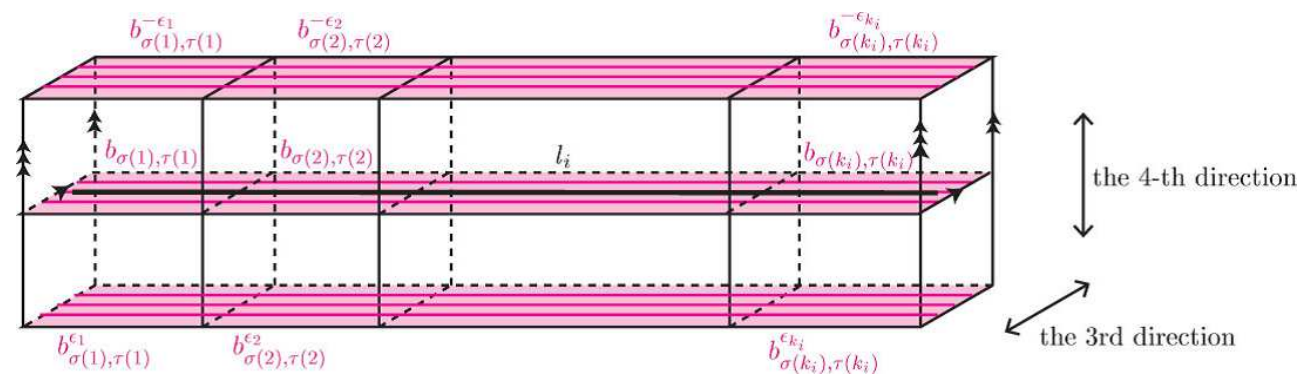

Figure 43. The solid torus $\pi^{-1}\left(l_{i}\right)$ in the case where $\rho^{-1}\left(l_{i}\right)$ is an annulus. In this figure, $\epsilon_{i}= \pm$.

Dehn filling on $X$ along a leaf of the foliation on $\bigcup_{i=1}^{k_{i}} b_{\sigma(i), \tau(i)}^{\epsilon_{i}}$, which lies in the torus $T_{i}=\partial \pi^{-1}\left(l_{i}\right) \subset \partial X$. Moreover, putting the gleam $g_{i} \in \mathbb{Z}$ on $R_{i}$ in this process corresponds to performing Dehn filling on $X$ along the slope $s_{i}$ obtained from the leaf by $g_{i}$-th power of the Dehn twist about the meridian of the solid torus $\pi^{-1}\left(l_{i}\right)$. Note that this slope $s_{i}$ can be expressed by $g_{i}\left[\mu_{i}\right]+\left[\lambda_{i}\right] \in H_{1}\left(T_{i} ; \mathbb{Z}\right)$, where $\mu_{i}$ is a meridian of the solid 
torus $\pi^{-1}\left(l_{i}\right)$ and $\lambda_{i}$ is a leaf of the foliation on $\bigcup_{i=1}^{k_{i}} b_{\sigma(i), \tau(i)}^{\epsilon_{i}}$. If $\rho^{-1}\left(l_{i}\right)$ is a Möbius band, it lies in the solid torus $\pi^{-1}\left(l_{i}\right)$ as shown in Figure 44, In this case, the Möbius band

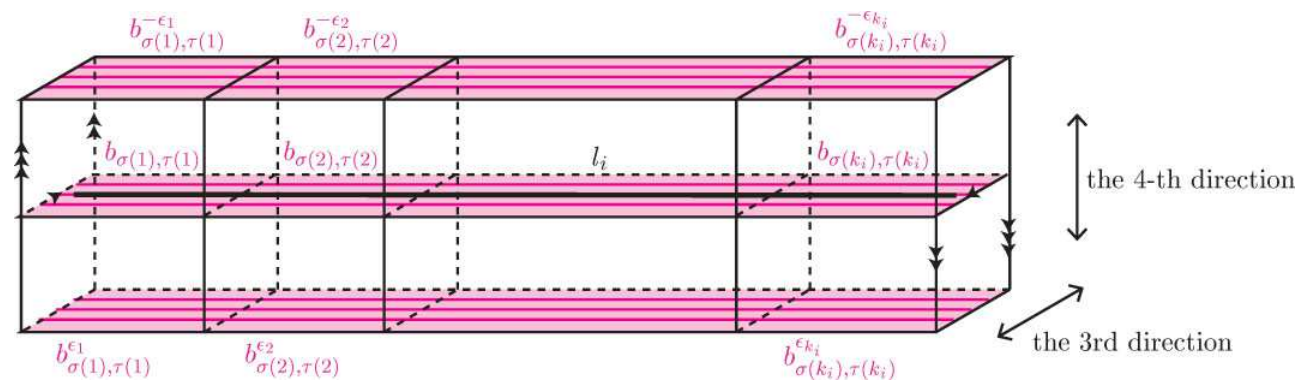

Figure 44 . The solid torus $\pi^{-1}\left(l_{i}\right)$ in the case where $\rho^{-1}\left(l_{i}\right)$ is a Möbius band.

$\bigcup_{i=1}^{k_{i}} b_{\sigma(i), \tau(i)}$ is no longer parallel to the boundary of the solid torus $\pi^{-1}\left(l_{i}\right)$. Let $a_{\sigma(1), \tau(1)}^{ \pm}$ be a foliated square shown in Figure 45. Then the union $a_{\sigma(1), \tau(1)}^{ \pm} \cup\left(\bigcup_{i=2}^{k_{i}} b_{\sigma(i), \tau(i)}\right)$ is
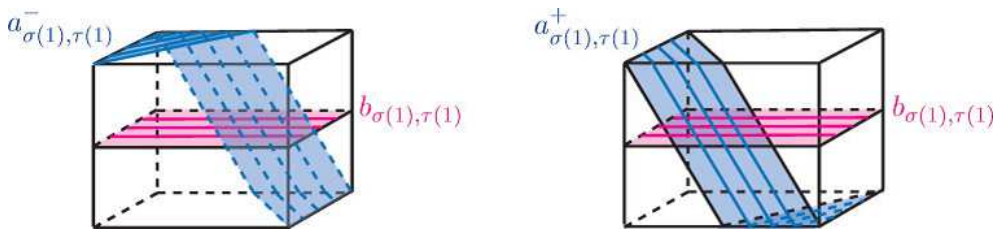

FiguRE 45. The foliation when the gleam is a half-integer.

an annulus that is isotopic to $\bigcup_{i=1}^{k_{i}} b_{\sigma(i), \tau(i)}^{\epsilon_{i}}$ after a $\pm 1 / 2$-twist. Hence again by the definition of gleams, attaching the disk $D_{i}$ to $P^{\prime}$ along $l_{i}$ and putting the gleam $\pm 1 / 2$ on the resulting region $R_{i}$ correspond to performing Dehn filling on $X$ along a leaf of the foliation on $a_{\sigma(1), \tau(1)}^{ \pm} \cup\left(\bigcup_{i=2}^{k_{i}} b_{\sigma(i), \tau(i)}\right)$. Moreover, putting the gleam $g_{i} \in\left(\frac{1}{2} \mathbb{Z}\right) \backslash \mathbb{Z}$ on $R_{i}$ in this process corresponds to performing Dehn filling on $X$ along the slope $s_{i}$ obtained from a leaf of $a_{\sigma(1), \tau(1)}^{+} \cup\left(\bigcup_{i=2}^{k_{i}} b_{\sigma(i), \tau(i)}\right)$ by $\left(g_{i}-1 / 2\right)$-th power of Dehn twist about the meridian of the solid torus $\pi^{-1}\left(l_{i}\right)$. Again, note that this slope $s_{i}$ can be written as $\left(g_{i}-1 / 2\right)\left[\mu_{i}\right]+\left[\lambda_{i}\right] \in H_{1}\left(T_{i} ; \mathbb{Z}\right)$, where $\mu_{i}$ is a meridian of the solid torus $\pi^{-1}\left(l_{i}\right)$ and $\lambda_{i}$ is a leaf of the foliation on $a_{\sigma(1), \tau(1)}^{+} \cup\left(\bigcup_{i=2}^{k_{i}} b_{\sigma(i), \tau(i)}\right)$.

As is explained in [12, we can equip the interior of $X$ with a complete, finite volume hyperbolic structure by regarding $X$ as $2 c(P)$ regular ideal octahedra glued by isometries. The decomposition of the interior of $X$ into these ideal octahedra exactly corresponds to the decomposition described in Figure 42. We recall that the regular ideal octahedron has a maximal horocusp section consisting of six Euclidean unit squares. This maximal horocusp gives rise to a maximal horoball neighborhood $C$ of the interior of $X$. By the above argument, the length of the slope $s_{i}$ with respect to $C$ coincides with $\operatorname{sl}\left(R_{i}\right)$. 


\section{ACKnowledgments}

The authors wish to express their gratitude to Francesco Costantino, Bruno Martelli and Osamu Saeki for very helpful suggestions and comments. This work was carried out while the second-named author was visiting Università di Pisa as a JSPS Postdoctoral Fellow for Reserch Abroad. He is grateful to the university and its staffs for the warm hospitality.

\section{REFERENCES}

[1] Agol, I., Lower bounds on volumes of hyperbolic Haken 3-manifolds, arXiv:math/9906182

[2] Agol, I., Bounds on exceptional Dehn filling, Geom. Topol. 4 (2000), 431-449.

[3] Agol, I., Storm, P. A., Thurston, W. P., Lower bounds on volumes of hyperbolic Haken 3manifolds. With an appendix by Nathan Dunfield, J. Amer. Math. Soc. 20 (2007), 1053-1077.

[4] Benedetti, R., Petronio, C., Branched standard spines of 3-manifolds, Lecture Notes in Mathematics 1653, Springer-Verlag, Berlin, 1997.

[5] Burlet, O., de Rham, G., Sur certaines applications génériques d'une variété close à 3 dimensions dans le plan, Enseignement Math. (2) 20 (1974), 275-292.

[6] Callahan, P. J., Dean, J. C., Weeks, J. R., The simplest hyperbolic knots, J. Knot Theory Ramifications 8 (1999) 279-297.

[7] Costantino, F., Shadows and branched shadows of 3 and 4-manifolds, Edizioni della Normale, Scuola Normale Superiore, Pisa, Italy, 2005.

[8] Costantino, F., A short introduction to shadows of 4-manifolds, Fund. Math. 188 (2005), 271291.

[9] Costantino, F., Stein domains and branched shadows of 4-manifolds, Geom. Dedicata 121 (2006), 89-111.

[10] Costantino, F., Branched shadows and complex structures of 4-manifolds, J. Knot Theory Ramifications 17 (2008), 1429-1454.

[11] Costantino, F., Frigerio, R., Martelli, B., Petronio, C., Triangulations of 3-manifolds, hyperbolic relative handlebodies, and Dehn filling, Comment. Math. Helv. 82 (2007), 903-933.

[12] Costantino, F., Thurston, D., 3-manifolds efficiently bound 4-manifolds, J. Topol. 1 (2008), 703-745.

[13] Endoh, M., Ishii, I., A new complexity for 3-manifolds, Japan. J. Math. (N.S.) 31 (2005), 131156.

[14] Futer, D., Kalfagianni, E., Purcell, J. S., Dehn filling, volume, and the Jones polynomial, J. Differential Geom. 78 (2008), 429-464.

[15] Gromov, M., Singularities, expanders and topology of maps. I. Homology versus volume in the spaces of cycles, Geom. Funct. Anal. 19 (2009), 743-841.

[16] Ikeda, H., Acyclic fake surfaces, Topology 10 (1971), 9-36.

[17] Ishii, I., Flows and spines, Tokyo J. Math. 9 (1986), 505-525.

[18] Kalmár, B., Stipsicz, A. I., Maps on 3-manifolds given by surgery, Pacific J. Math. 257 (2012), 9-35.

[19] Kapovich, M., Hyperbolic manifolds and discrete groups, Progress in Mathematics 183, Birkhäuser Boston Inc., Boston, MA, 2001.

[20] Koda, Y., Branched spines and Heegaard genus of 3-manifolds, Manuscripta Math. 123 (2007), 285-299.

[21] Kushner, L., Levine, H., Porto, P., Mapping three-manifolds into the plane. I, Bol. Soc. Mat. Mexicana (2) 29 (1984), 11-33.

[22] Lackenby, M., Word hyperbolic Dehn surgery, Invent. Math. 140 (2000), 243-282.

[23] Levine, H., Elimination of cusps, Topology 3 (1965), 263-296.

[24] Levine, H., Classifying immersions into $\mathbb{R}^{4}$ over stable maps of 3 -manifolds into $\mathbb{R}^{2}$, Lecture Notes in Mathematics 1157, Springer-Verlag, Berlin, 1985. 
[25] Martelli, B., Links, two-handles, and four-manifolds, Int. Math. Res. Not. IMRN 2005, 35953623.

[26] Martelli, B., Four-manifolds with shadow-complexity zero, Int. Math. Res. Not. IMRN 2011, $1268-1351$.

[27] Matveev, S. V., Algorithmic Topology and Classification of 3-Manifolds, Algorithms Comput. Math. 9, Springer, Berlin, 2003.

[28] Morgan, J. W., On Thurston's uniformization theorem for three-dimensional manifolds, The Smith conjecture (New York, 1979), pp. 37-125, Pure Appl. Math. 112, Academic Press, Orlando, FL, 1984.

[29] Otal, J-P., Le théorème d'hyperbolisation pour les variétés fibrées de dimension 3, Astérisque 235, 1996.

[30] Otal, J-P., Thurston's hyperbolization of Haken manifolds, Surveys in differential geometry, Vol. III (Cambridge, MA, 1996), pp. 77-194, Int. Press, Boston, MA, 1998.

[31] Perelman, G., The entropy formula for the Ricci flow and its geometric applications, arXiv:math/0211159.

[32] Perelman, G., Ricci flow with surgery on three-manifolds, arXiv:math/0303109.

[33] Perelman, G., Finite extinction time for the solutions to the Ricci flow on certain three-manifolds, arXiv:math/0307245

[34] Petronio, C., Generic flows on 3-manifolds, arXiv:1211.6445.

[35] Rolfsen, D., Knots and links, Mathematics Lecture Series 7, Publish or Perish, Inc., Berkeley, Calif., 1976.

[36] Saeki, O., Simple stable maps of 3-manifolds into surfaces, Topology 35 (1996), 671-698.

[37] Saeki, O., Topology of singular fibers of differentiable maps, Lecture Notes in Mathematics 1854, Springer-Verlag, Berlin, 2004.

[38] Thurston, D., The algebra of knotted trivalent graphs and Turaev's shadow world, Invariants of knots and 3-manifolds (Kyoto, 2001), pp. 337-362, Geom. Topol. Monogr. 4, Geom. Topol. Publ., Coventry, 2002.

[39] Thurston, W. P., The geometry and topology of three-manifolds, lecture notes, Princeton University, Princeton, 1980, available at http://msri.org/publications/books/gt3m/

[40] Thurston, W. P., Three-dimensional manifolds, Kleinian groups and hyperbolic geometry, Bull. Amer. Math. Soc. (N.S.) 6 (1982), 357-381.

[41] Turaev, V. G., Shadow links and face models of statistical mechanics, J. Differential Geom. 36 (1992), 35-74.

[42] Turaev, V. G., Quantum invariants of knots and 3-manifolds, de Gruyter Studies in Mathematics 18, Walter de Gruyter \& Co., Berlin, 1994.

[43] Yoshida, K., The minimal volume orientable hyperbolic 3-manifold with 4 cusps, arXiv:1209.1374

Tohoku University, Sendai, 980-8578, JaPAN

E-mail address: ishikawa@m.tohoku.ac.jp

Mathematical Institute

TOHOKu University, Sendai, 980-8578, Japan

AND

(Temporary) Dipartimento di Matematica

Università di Pisa, Largo Bruno Pontecorvo 5, 56127 Pisa, Italy

E-mail address: koda@math.tohoku.ac.jp 